\title{
ON THE FATIGUE AND FRACTURE OF BLADED AND INTEGRALLY BLADED ROTORS OF AIRCRAFT ENGINE COMPRESSORS
}

\author{
by \\ Dikran Mangardich \\ Bachelor of Aerospace Engineering, Ryerson University (2017)
}

\author{
A thesis \\ presented to Ryerson University \\ in partial fulfillment of the \\ requirements for the degree of \\ Master of Applied Science \\ in the program of \\ Aerospace Engineering
}

Toronto, Ontario, Canada, 2019

(C) Dikran Mangardich, 2019 


\section{AUTHOR'S DECLARATION FOR ELECTRONIC SUBMISSION OF A THESIS}

I hereby declare that I am the sole author of this thesis. This is a true copy of the thesis, including any required final revisions, as accepted by my examiners.

I authorize Ryerson University to lend this thesis to other institutions or individuals for the purpose of scholarly research.

I further authorize Ryerson University to reproduce this thesis by photocopying or by other means, in total or in part, at the request of other institutions or individuals for the purpose of scholarly research. I understand that my thesis may be made electronically available to the public. 


\title{
ON THE FATIGUE AND FRACTURE OF BLADED AND INTEGRALLY BLADED ROTORS OF AIRCRAFT ENGINE COMPRESSORS
}

\author{
Dikran Mangardich \\ Master of Applied Science, Aerospace Engineering, Ryerson University, Toronto (2019)
}

\begin{abstract}
The fatigue and fracture for bladed and integrally bladed rotors (IBR) of aircraft engine compressors has been studied. For IBRs, a new distinct finite element technique was developed to model crack propagation under combined low cycle and high cycle fatigue loading. The crack trajectory, aspect ratio, and shape resulting from the method agreed very well with airfoils which fractured in service. The technique can be extended on other compressor disk applications. For bladed rotors limited by fretting fatigue, a unique fracture mechanics based methodology was developed for obtaining an evolved coefficient of friction (COF) resulting from fretting motion between the fan blade and hub. The predicted nucleation location, nucleation life, crack trajectory, shape and propagation life agreed well with the fractured components. The study confirms that the fretting-specific modified Smith-Watson-Topper (SWT) parameter more accurately predicts the nucleation location and life of the crack compared to the plain fatigue SWT parameter.
\end{abstract}




\section{ACKNOWLEDGEMENTS}

I would first like to thank my direct thesis advisor Dr. Fawaz of the Aerospace Engineering Department at Ryerson University for his continual guidance, advice, and for steering me in the right direction not only in my studies but outside of academia. I am especially grateful of Dr. Fawaz for being the catalyst of my young aerospace engineering career.

I would like to express my gratitude to $\mathrm{P} \& \mathrm{WC}$ as well as my direct manager at $\mathrm{P} \& \mathrm{WC}$, Dr. Abrari for providing me with the opportunity of working full-time while pursuing a Master Degree in a field of study that I greatly value. Dr. Abrari provided me with the time, resources, and technical support required to complete my thesis. My academic success would not have been possible without his tremendous support.

I would also like to thank Bruce J. Carter from Fracture Analysis Consultants (FAC) for his technical support, to P\&WC`s Materials Investigations department for conducting the fractographic investigations, and to the Component Dynamics department for the modal analysis and NSMS testing.

Finally, I must express my very profound gratitude to my family, fiancé and close friends for their continual support and for guiding me in difficult situations. Without their help, I would not reach where I have today.

Thank You All! 


\section{TABLE OF CONTENTS}

AUTHOR'S DECLARATION FOR ELECTRONIC SUBMISSION OF A THESIS ............................................... ii

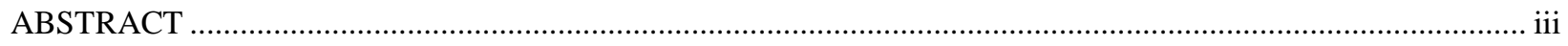

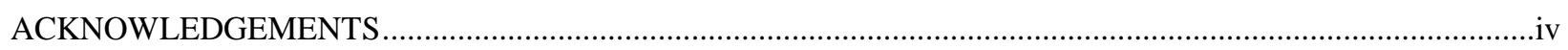

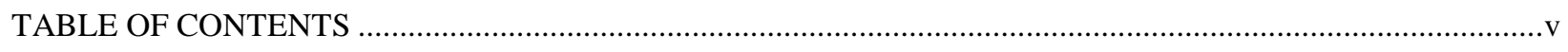

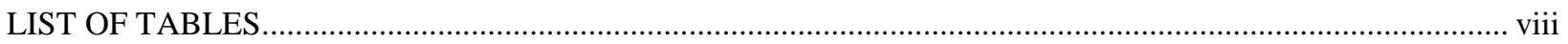

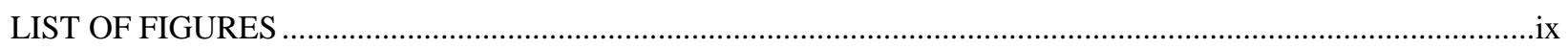

LIST OF ABBREVIATIONS AND SYMBOLS ……...................................................................................

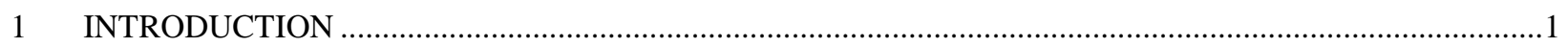

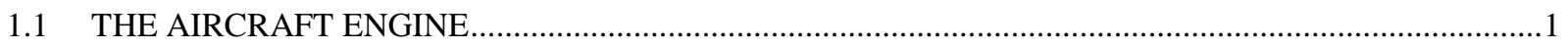

1.2 FATIGUE OF BLADED AND INTEGRALLY BLADED COMPRESSOR ROTORS …...........................2

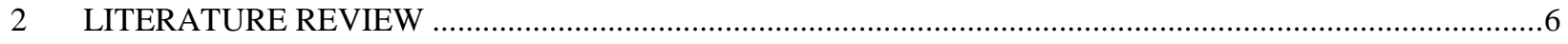

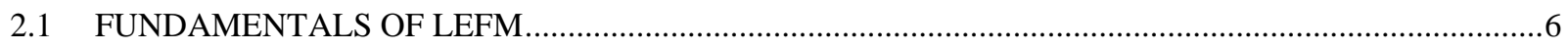

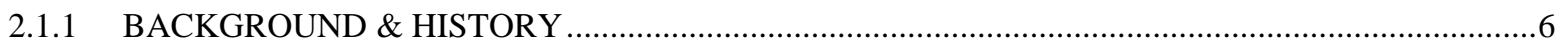

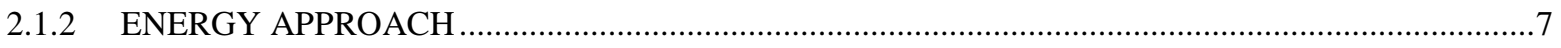

2.1.2.1 LOAD CONTROL VS DISPLACEMENT CONTROL …............................................................

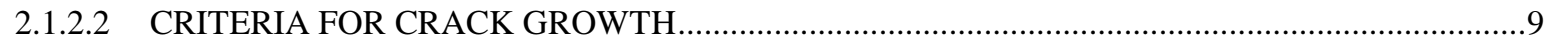

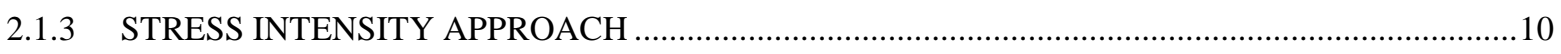

2.1.3.1 ANALYTICAL CLOSED FORM SOLUTIONS OF STRESS INTENSITY FACTORS ................12

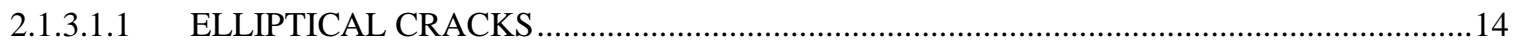

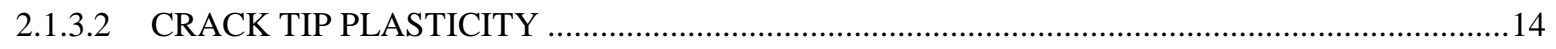

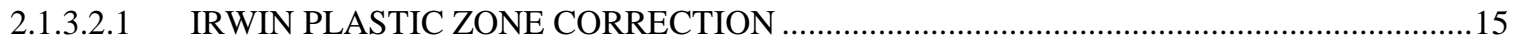

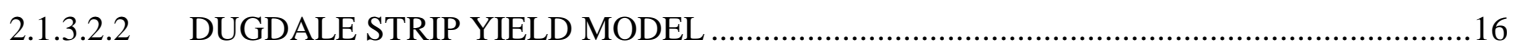

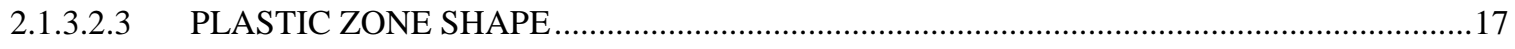

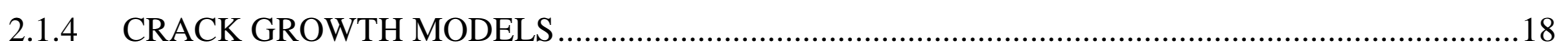

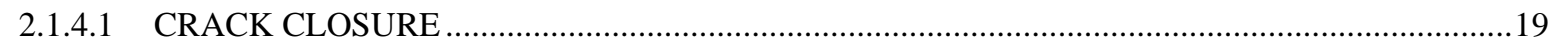

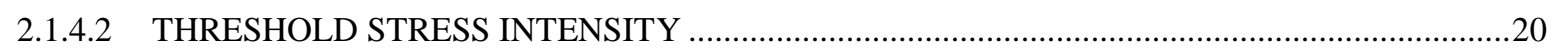

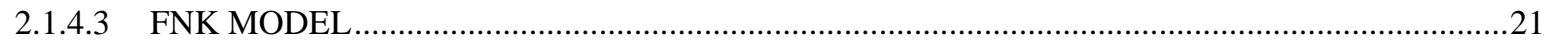

2.1.5 BRIEF REVIEW OF ELASTIC PLASTIC FRACTURE MECHANICS ……..................................22 


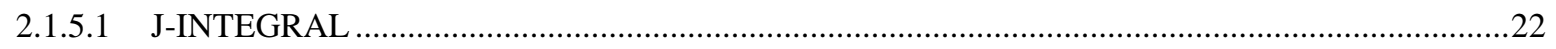

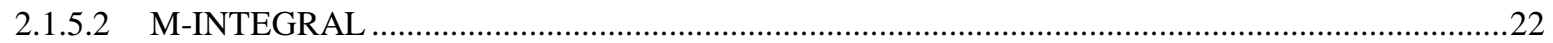

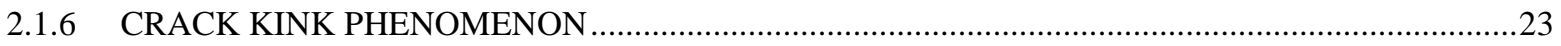

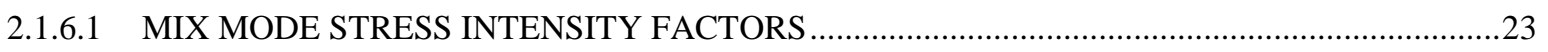

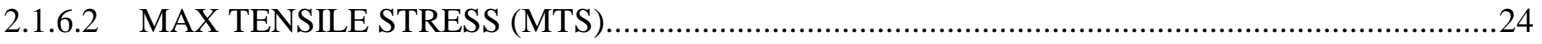

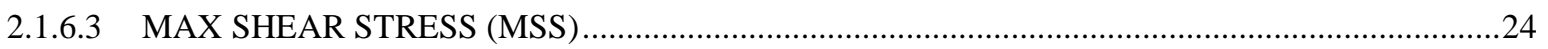

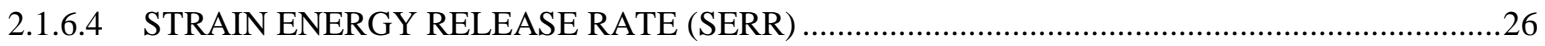

2.1.6.5 MODIFIED STRAIN ENERGY RELEASE RATE (MSERR) ……………...............................26

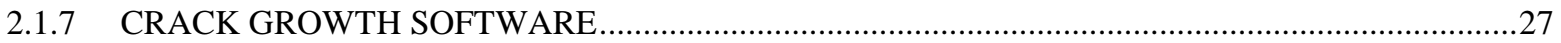

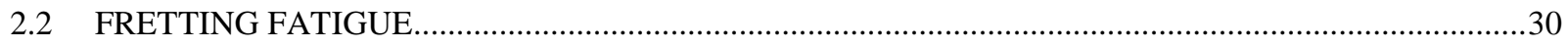

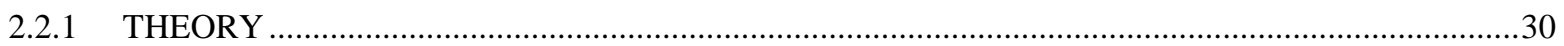

2.2.2 INVESTIGATIONS OF FRETTING FATIGUE FAILURES IN AIRCRAFT ENGINES ..................33

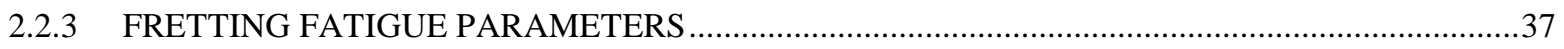

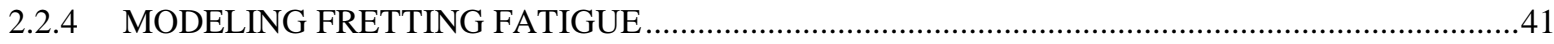

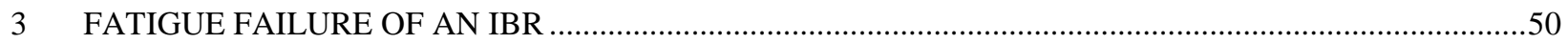

3.1 FRACTOGRAPHIC INVESTIGATIONS OF FRACTURED COMPRESSOR BLADES .........................50

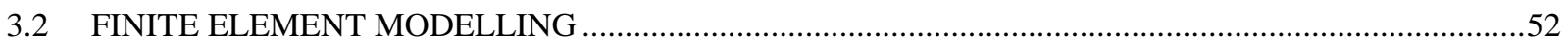

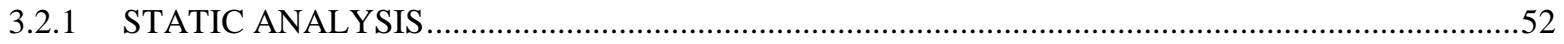

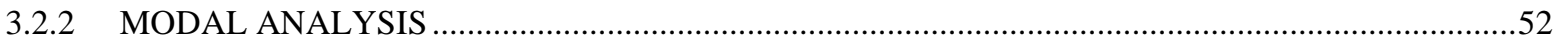

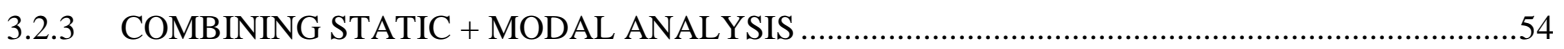

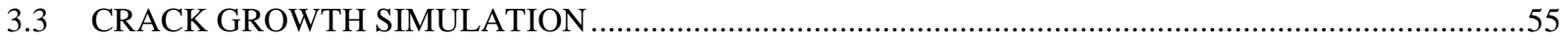

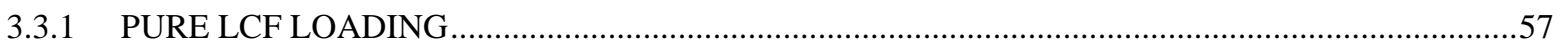

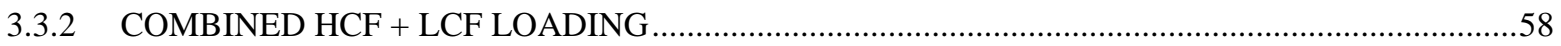

3.4 CRACK PROPAGATION LIFE FOR THE LCF + HCF SIMULATION …….............................................61

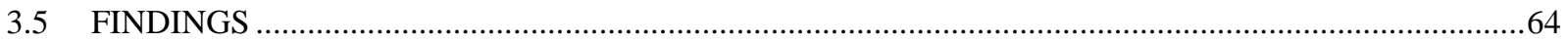

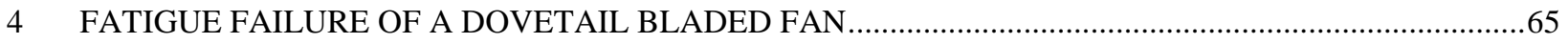

4.1 SPIN TESTING AND FRACTOGRAPHIC INVESTIGATIONS ……................................................65

4.2 FINITE ELEMENT MODELING AND SUBMODELING ……............................................................68

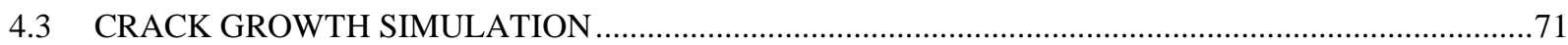




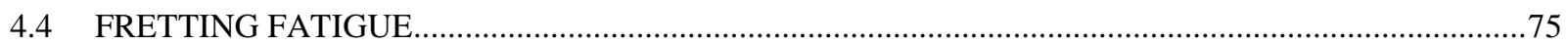

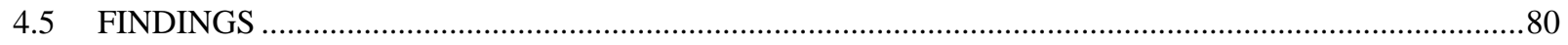

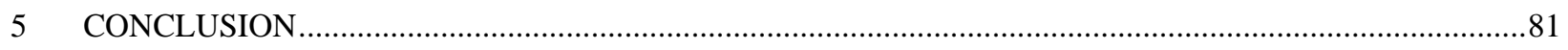

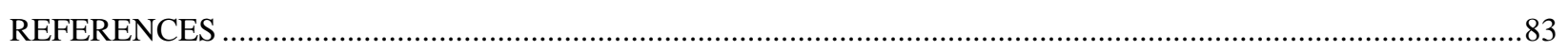




\section{LIST OF TABLES}

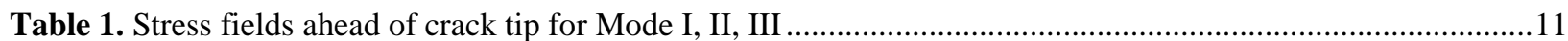

Table 2. Geometry corrections for common test specimen [37] ....................................................................13 


\section{LIST OF FIGURES}

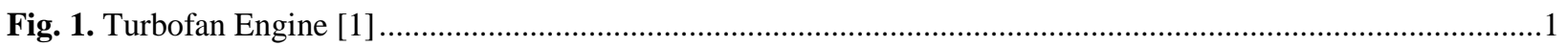

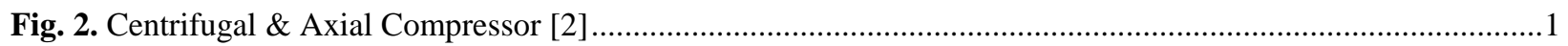

Fig. 3. (a) Typical bladed-disk rotor (dovetail joint) [3] (b) Axial IBR compressor .................................................2

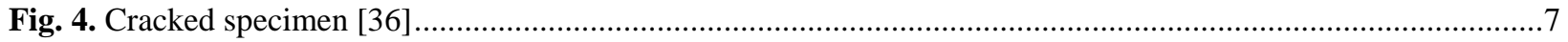

Fig. 5. Load controlled vs Displacement Controlled specimen ...................................................................... 8

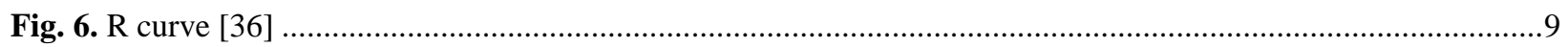

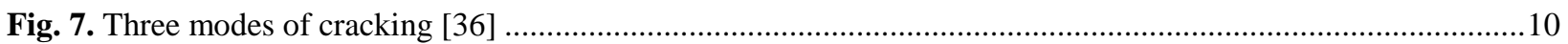

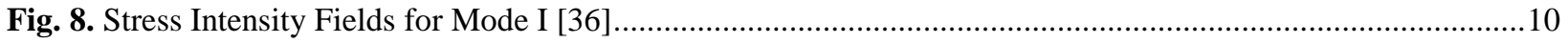

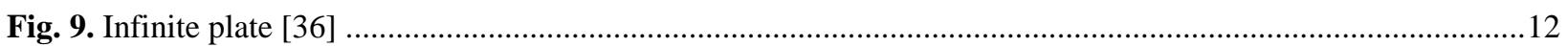

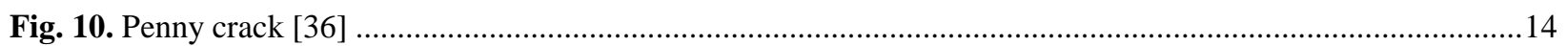

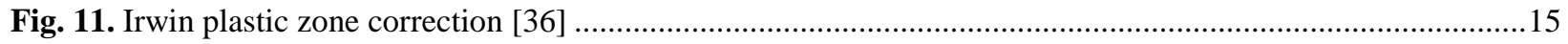

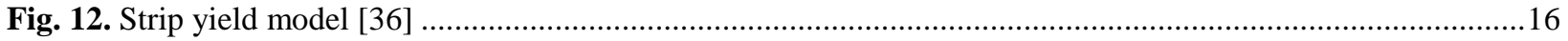

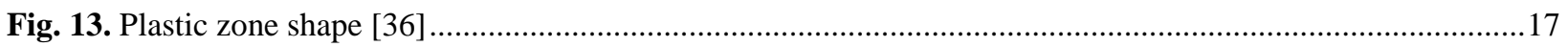

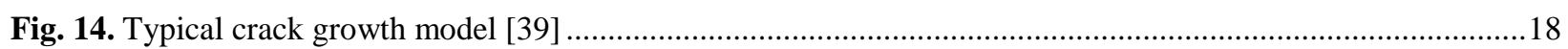

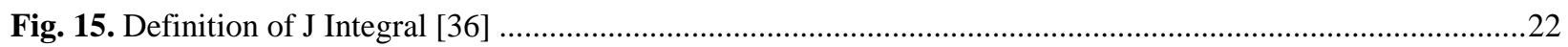

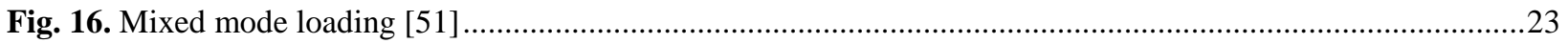

Fig. 17. MTS \& MSS for a) KI/KII not zero and b) KI/KII=0 [51] .............................................................25

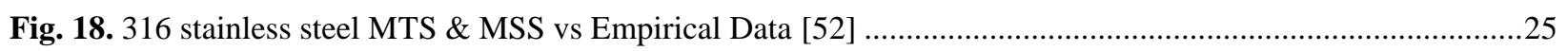

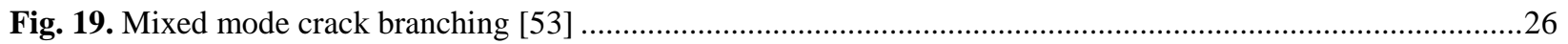

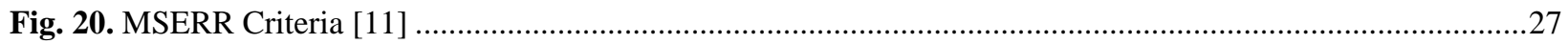

Fig. 21. - Corner flaw crack propagation using Sigma-Max method and planar extension method [8] .....................27

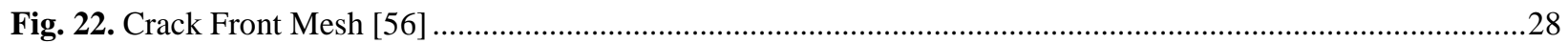

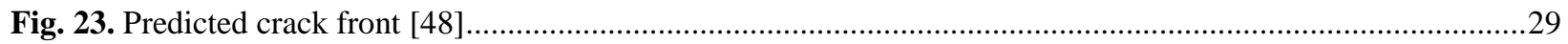

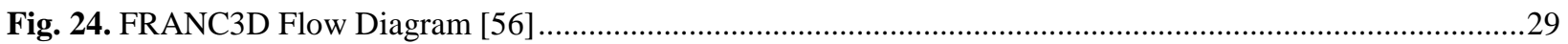

Fig. 25. Benchmark for penny crack, FRANC3D vs analytical Sneddon [57] .....................................................22

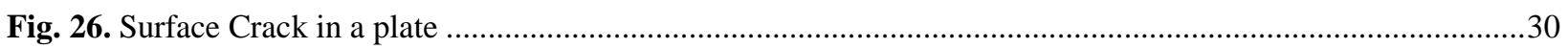

Fig. 27. (a) Typical bladed-disk rotor (dovetail joint) [3] (b) Forces acting at dovetail interface [58] .......................31

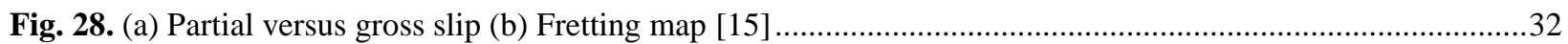

Fig. 29. 1 st stage compressor assembly with failed pinhole lug [20] ....................................................................34

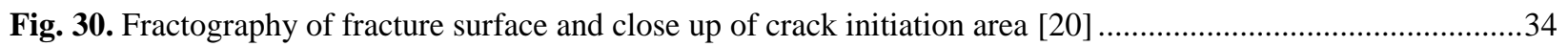

Fig. 31. Visual fracture appearance of the two low pressure turbine blades [21] ......................................................34

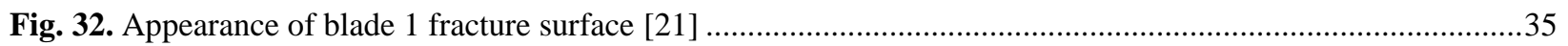

Fig. 33. (a) Brownish marks on fir tree root (b) High magnification revealing fretting cracks [22] .........................35

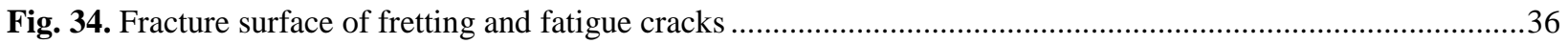

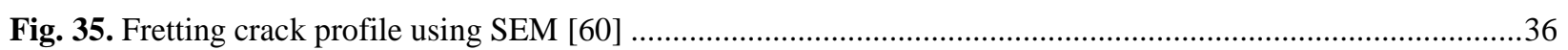

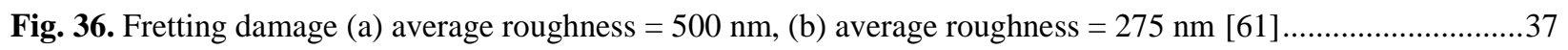


Fig. 37. Crack growth path in tested specimen [24]

Fig. 38. Interface stresses for $20 \mathrm{kN}$ loading [30] ......

Fig. 39. (a) Damage due to fretting (b) Predicted fretting life according to modified SWT approach [33] ...............40

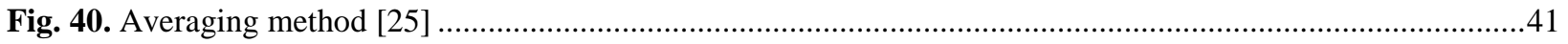

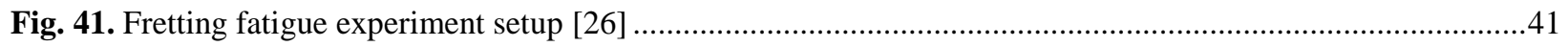

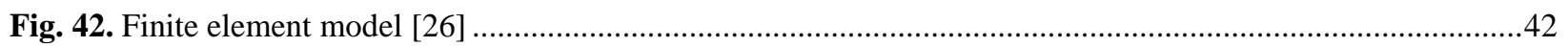

Fig. 43. Procedure used to find maximum SWT value [27] ...........................................................................

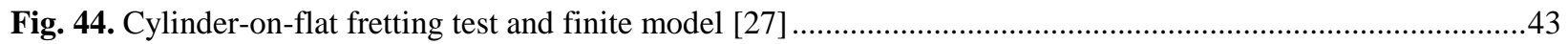

Fig. 45. (a) Shear traction distribution at different load steps (b) subsurface stress distributions at step 2 [27] .........44

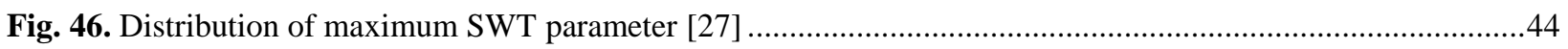

Fig. 47. (a) Shear versus normal contact force (b) Comparison of pressure tractions for various mesh sizes [62] .....45

Fig. 48. (a) Failed blade specimens (b) Stress intensity along crack depth for various friction coefficients [58] .......46

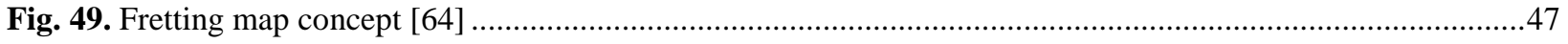

Fig. 50. Fretting nucleation parameter for dovetail fan attachment [18] .............................................................48

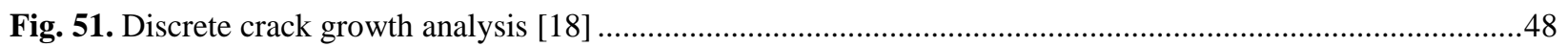

Fig. 52. Free surface crack on dovetail attachment fan blade and simulated crack growth [8] ................................49

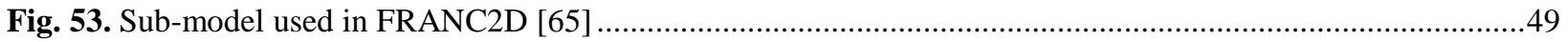

Fig. 54. Macro view of a fractured high pressure compressor airfoil, $53.3 \mathrm{~mm}$ chord length, $4.3 \mathrm{~mm}$ thickness .......50

Fig. 55. Airfoil fracture surfaces of a high pressure compressor airfoil for events A, B, C from left to right............50

Fig. 56. Fracture surface showing crack origin (red), river lines (yellow), and beach marks (green) - Event C ..........51

Fig. 57. Crack initiation site. No anomalies were found through SEM and EDS - Event C ...................................51

Fig. 58. SEM showing river lines cross multiple progression marks at TE \& LE, characteristic of HCF - Event C ..51

Fig. 59. (a) Max principal stress near airfoil release at maximum takeoff conditions (b) Stress strain curve .............52

Fig. 60. Blade deflection for dominant bending mode (a) tension on pressure side (b) tension on suction side .........53

Fig. 61. Resulting blade root max principal (a) tension on pressure side (b) tension on suction side .......................53

Fig. 62. NSMS measured tip deflections at leading edge in percent span for various operating conditions ...............54

Fig. 63. FE results for peak steady and dynamic stress locations and crack locations of events A, B, and C ............54

Fig. 64. (a) Tip bending force and max principal stress (b) tension at pressure side (c) tension at suction side ..........55

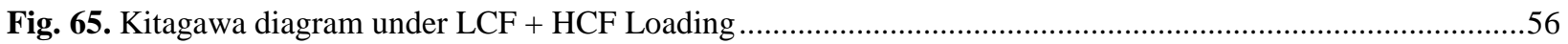

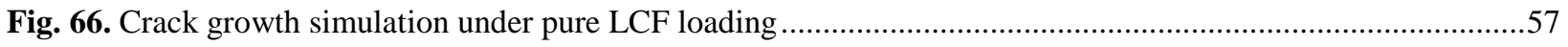

Fig. 67. Mode I and II stress intensities along first 55 crack fronts under pure LCF loading $(A=T E, B=L E)$........58

Fig. 68. Crack growth simulation under combined HCF + LCF loading ............................................................58

Fig. 69. Mode I and II stress intensities along first 55 crack fronts under LCF \& HCF Loading (A = TE, B = LE) ..60

Fig. 70. - Mode I stress intensities along crack front corresponding to crack depth of $2.5 \mathrm{~mm}$............................60

Fig. 71. LCF - HCF flight block showing a typical mission of the compressor rotor ..............................................62

Fig. 72. Mode I SIF along crack depth under LCF+HCF Tension and LCF+HCF Compression .............................62

Fig. 73. (a) Crack growth life sensitivity to transient HCF cycle accumulation per flight (b) HCF cycles to failure .64 
Fig. 74. (a) Two-blade sector (b) inspection locations on hub (c) inspection locations of the blade...... 66

Fig. 75. Representative load curve used for the spinning tests.....

Fig. 76. Four cracks detected from three individual spin tests concentrated at LE pressure side slot ....................67

Fig. 77. Severe fretting along slot surface (Crack A) present on all slots at both the leading and trailing edges .......67

Fig. 78. Striation counts on the opened fracture surfaces for Cracks A-D using EDS and micrometer readings.......68

Fig. 79. (a) Two-bladed sector FE model. Max principal stress plot for hub for (b) $\mathrm{COF}=0.35$, (c) $\mathrm{COF}=0.70 \ldots \ldots . .69$

Fig. 80. (a) Path (b) to (e) Principal, radial, hoop and shear stresses along path for COF of 0.70 and $0.35 \ldots \ldots \ldots \ldots . . .70$

Fig. 81. (a) Coarsened submodel consisting of the hub, blade (b) Stresses along crack propagation path................70

Fig. 82. Initial semi-circular shaped crack with radius $125 \mu \mathrm{m}$ inserted at the LE pressure side ............................73

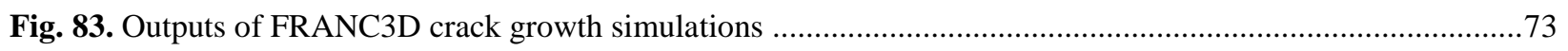

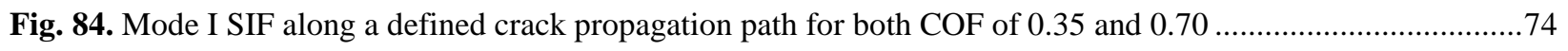

Fig. 85. Predicted crack growth rates compared to test measurements .......................................................74

Fig. 86. Crack propagation lives of simulations compared with experimental..............................................75

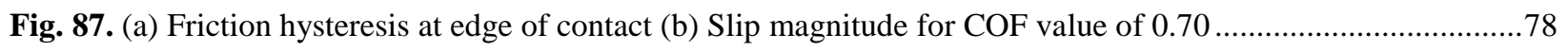

Fig. 88. SWT parameter calculated at (a) Pressure side and (b) Suction side ...................................................78

Fig. 89. Modified SWT parameter calculated at (a) Pressure side and (b) Suction side......................................79

Fig. 90. (a) Fretting nucleation life calculated at the critical LE pressure side location (b) $D f r e t$ curve .................79 


\section{LIST OF ABBREVIATIONS AND SYMBOLS}

\begin{tabular}{|c|c|}
\hline $\mathrm{COF}$ & Coefficient of Friction \\
\hline EDS & Energy Dispersive Spectrometer \\
\hline EPFM & Elastic Plastic Fracture Mechanics \\
\hline FE & Finite Element \\
\hline FNK & Forman-Newman-de Koning \\
\hline FOD & Foreign Object Damage \\
\hline FPI & Fluorescent Penetrant Inspection \\
\hline FS & Fatemi-Socie \\
\hline FTIR & Fourier Transform Infrared Spectroscopy \\
\hline $\mathrm{HCF}$ & High Cycle Fatigue \\
\hline IBR & Integrally Bladed Rotor \\
\hline LCF & Low Cycle Fatigue \\
\hline $\mathrm{LE}$ & Leading Edge \\
\hline LEFM & Linear Elastic Fracture Mechanics \\
\hline MSERR & Modified Strain Energy Release Rate \\
\hline MSS & Max Shear Stress \\
\hline MTS & Max Tensile Stress \\
\hline NSMS & Non-Intrusive Stress Measurement Systems \\
\hline RPM & Revolutions Per Minute \\
\hline SEM & Scanning Electron Microscope \\
\hline SERR & Strain Energy Release Rate \\
\hline SIF & Stress Intensity Factor \\
\hline SWT & Smith-Watson-Topper \\
\hline $\mathrm{TE}$ & Trailing Edge \\
\hline TP & Transient Pass-Through \\
\hline $\mathrm{F}$ & Total energy in system due to work performed by an external force \\
\hline $\mathrm{U}$ & Elastic energy stored in the system \\
\hline $\mathrm{W}$ & Work required to break bonds and create new surfaces \\
\hline $\mathrm{U}$ & Strain energy in system due to work done by an external force \\
\hline$\sigma$ & Applied nominal stress \\
\hline$a$ & Depth of crack in specimen \\
\hline$B$ & Width of specimen \\
\hline$E$ & Elastic modulus \\
\hline$\Pi$ & Potential energy available for crack growth \\
\hline G & Total energy release rate for an extension of unit area crack surface \\
\hline A & Crack surface area \\
\hline
\end{tabular}




\begin{tabular}{|c|c|}
\hline $2 \gamma_{\mathrm{s}}$ & Energy required to generate two new crack surfaces \\
\hline$\sigma_{\mathrm{f}}$ & Stress at failure due to fracture \\
\hline$\gamma_{\mathrm{p}}$ & Modification to account for plastic deformation ahead of plastic zone \\
\hline $\mathrm{G}_{\mathrm{I}}$ & Strain energy release rate due to Mode I loading \\
\hline $\mathrm{G}_{\mathrm{II}}$ & Strain energy release rate due to Mode II loading \\
\hline $\mathrm{G}_{\text {III }}$ & Strain energy release rate due to Mode III loading \\
\hline $\mathrm{K}_{\mathrm{I}}$ & Mode I Stress Intensity Factor \\
\hline $\mathrm{K}_{\mathrm{II}}$ & Mode II Stress Intensity Factor \\
\hline $\mathrm{K}_{\text {III }}$ & Mode III Stress Intensity Factor \\
\hline $\mathrm{P}$ & Applied load on cracked specimen \\
\hline$\Delta$ & Displacement resulting from load on cracked specimen \\
\hline c & Compliance variable \\
\hline$\sigma_{y s}$ & Yield stress of material \\
\hline$r_{p}$ & Plastic zone radius \\
\hline$\frac{d a}{d N}$ & Fatigue crack growth rate \\
\hline$\Delta K$ & Stress Intensity Range \\
\hline$R$ & Stress ratio \\
\hline$\Delta K_{t h}$ & Fatigue threshold stress intensity range \\
\hline$J$ & J-Integral method \\
\hline$M$ & M-Integral method \\
\hline$\theta$ & Angle ahead of crack tip \\
\hline$\Delta \varepsilon$ & Normal strain range \\
\hline$\Delta \gamma$ & Shear strain range \\
\hline $2 N_{f}$ & Fatigue cycles to failure \\
\hline$\tau$ & Shear stress \\
\hline$\delta$ & Relative tangential motion caused by fretting \\
\hline$D_{\text {fret }}$ & Damage parameter due to frictional wear \\
\hline$\tau \delta$ & Frictional work due to fretting \\
\hline$(\tau \delta)_{t h}$ & Frictional work material threshold \\
\hline
\end{tabular}




\section{INTRODUCTION}

\subsection{THE AIRCRAFT ENGINE}

Turboprop, turbojet, and turbofan engines are the three main forms of propulsion systems used in the aviation industry. These engines each have their own distinct features but ultimately consist of common components such as the compressor, combustion chamber, and turbine to power the rotating shaft. In a turbofan configuration (Fig. 1), the rotating low-pressure spool connects the fan and low-pressure compressor stages to the low-pressure turbine. The high-pressure spool surrounds the low pressure spool and connects the high-pressure compressor to the high-pressure turbine. Due to its complex nature, the aircraft engine entails a multidisciplinary design process which involves the collaboration of aerodynamic, structural and performance, and air-oil-system engineers.

The focus of this work will be primarily within the compressor stage of the engine. The compressor of an aircraft engine consists of multiple stages of axial or centrifugal compressors which are connected to a driving turbine which transmits torque through a rotating shaft, commonly referred to as a spool. As the spool rotates, the oncoming air experiences an increase of kinetic energy due to an added component of velocity induced by the rotational speed of the compressor airfoils. A stator or diffuser redirects the fluid to the next stage. This interaction of the compressor and the stator increases the static pressure of the fluid before entrance to the combustor. High overall pressure ratios are often desirable but are limited by aerodynamic and mechanical considerations. For axial compressors, a single axial stage can have a pressure ratio of 2:1, whereas a centrifugal stage can have a pressure ratio of 10:1.

Aircraft engine compressors are commonly made of titanium alloys due to their metallurgical properties, availability, low life cycle cost, and ease of manufacturing. Compressors made of titanium alloys have excellent performance in the field due to their mechanical, corrosion and physical properties. More specifically, titanium alloys have excellent tensile strength to weight ratios and desirable fatigue properties. These mechanical properties are essential for ensuring the structural integrity of the compressor rotors under the extreme centrifugal loading and high temperatures experienced within the aircraft engine.

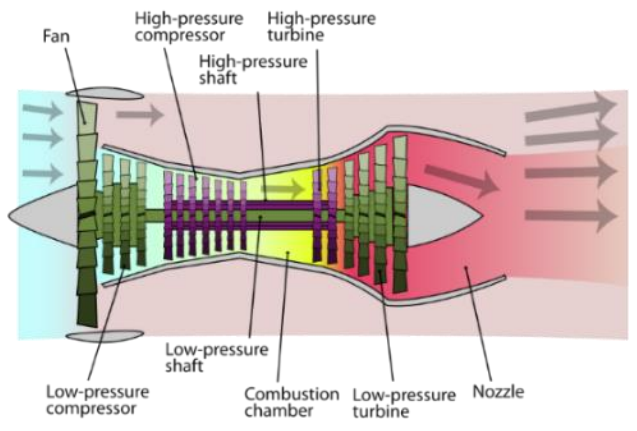

Fig. 1. Turbofan Engine [1]

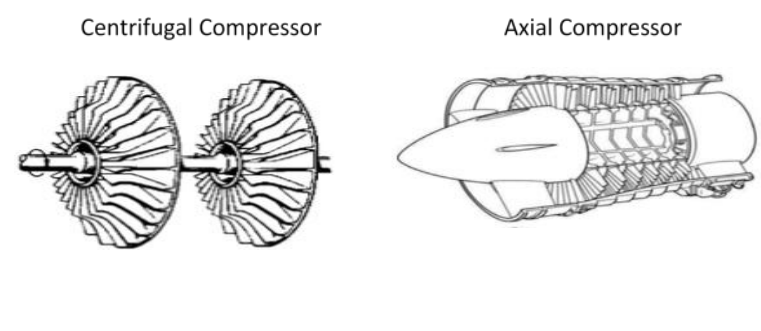

Fig. 2. Centrifugal \& Axial Compressor [2] 


\subsection{FATIGUE OF BLADED AND INTEGRALLY BLADED COMPRESSOR ROTORS}

There are two main types of axial compressor rotor designs as shown in Fig. 3. These are the bladed rotor and the IBR. For both designs, the primary objective is to produce a compressor that satisfies low cycle fatigue (LCF) and high cycle fatigue (HCF) requirements, is tolerant to foreign object damage (FOD), is aerodynamically efficient, and is reliable in the field while satisfying weight and cost constraints agreed by the customer. The LCF life of a compressor disks is a requirement defined by the customer and original equipment manufacturer in terms of number of hours or flights. Depending on the application, life requirements for compressors of civilian aircraft can range from 15,000 to 30,000 flight cycles. To ensure the rotors meet target life, the compressor disk must be optimized until an acceptable stress level is achieved at high power conditions for critical stress locations. The LCF life of compressor disks are determined in consideration of the aircraft mission defined by the customer, and the stress and temperature profile for critical life limiting locations. Stresses are obtained by conducting three-dimensional finite element analysis and temperature profiles are determined through transient thermal mission analysis. These inputs combined with location dependent life curves established through years of specimen and spin testing are used to substantiate the life of the compressor disk. The life curves used for lifing are location, material, and geometry dependent, thus multiaxial fatigue effects are accounted for in the predicted lives. For conservatism, the life curves are based on data reduction and Weibull probabilistic distribution which predicts the life for which one rotor out of one thousand results in a crack measuring $760 \mu \mathrm{m}$ (length) x $380 \mu \mathrm{m}$ (depth).
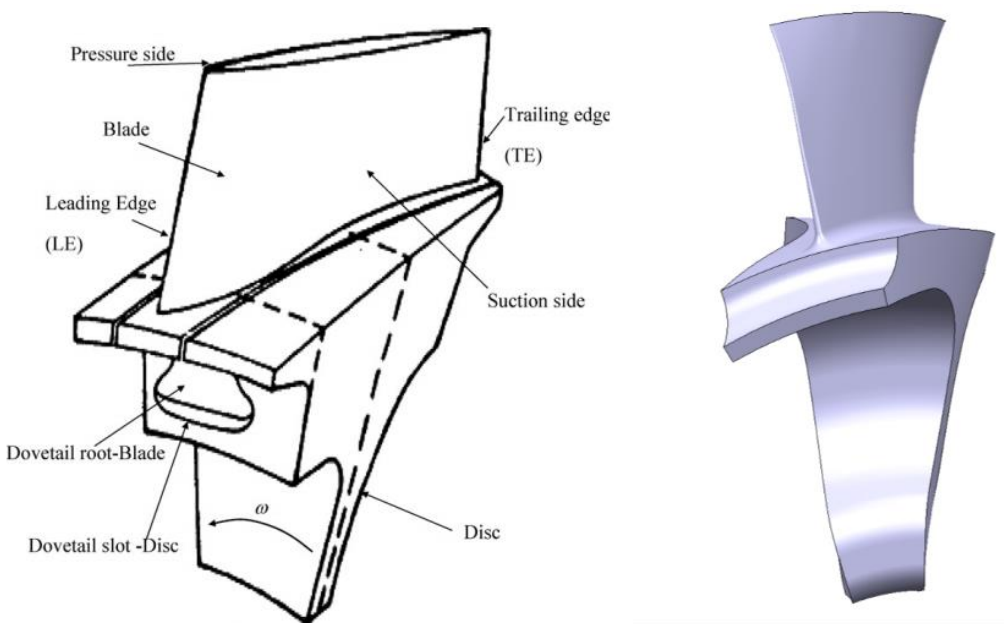

Fig. 3. (a) Typical bladed-disk rotor (dovetail joint) [3] (b) Axial IBR compressor

Compressor IBRs are subjected in flight operation to a steady stress associated with LCF as a result of centripetal, thermal and assembly loads, and vibratory stresses associated with HCF, occurring at resonance conditions [4]. HCF stresses arise from aerodynamic excitations, mechanical vibration, airfoil flutter, and acoustic excitation [5]. Once the useful life of a component is exhausted resulting in crack initiation, a damage tolerant approach is used to determine the crack propagation life, direction of crack growth, and the size and shape before final fracture. Byrne et al. [6] identified two regimes of crack growth. The first is a LCF growth regime, where the minor cycles associated with 
HCF loading do not contribute to the advance of the crack and have negligible crack growth rate. The second is a HCF crack growth onset regime, where a LCF driven crack grows large enough so that the applied stress intensity under resonant vibration is greater than the materials threshold stress intensity.

The use of linear elastic fracture mechanics (LEFM) in a crack propagation analysis is limited to the assumption that the stress and displacement fields surrounding the crack are uniquely described through a single fracture parameter such as the stress intensity field or K-field. However, aircraft engine components such as high-pressure compressors or turbines undergo high temperatures and stresses resulting in significant plastic regions surrounding the crack tip. In some cases, the plastic region can engulf the dominant K-field thus requiring advanced elastic-plastic fracture mechanics (EPFM) methods such as the J-Integral or M-integral which make use of a Ramberg-Osgood model for the material [7]. The use of the J-Integral or M-Integral is not limited to EPFM but can also be used for LEFM where the J-integral simplifies to the energy release rate.

Several works have used LEFM to simulate 3D crack growth of aircraft engine components. Barlow and Chandra [8] used FRANC3D, a 3D fracture mechanics simulation software, to simulate fatigue crack propagation of a military engine fan blade under pure centrifugal and aerodynamic loading. A free surface crack was analyzed on the contact surface of a fan blade attachment and allowed to grow using the max tensile theory (MTS). The MTS generated crack correlated well with field results. In this analysis, the dovetail fan blade attachment was sufficiently damped that the effects of dynamic vibratory stresses in the advancement of the crack was negligible. However, studies $[9,10]$ have shown that when present, the influence of vibratory stresses on the direction and shape of the crack could be significant such as a crack on an airfoil compressor IBR where resonant vibration has pronounced effects. However, there is uncertainty about the actual vibratory stress levels experienced by components and a lack of studies devoted to the numerical modeling of crack growth under mixed non-proportional combined LCF-HCF loading of compressor blades. Recently, Pettit et al. [11] demonstrated a method to predict the direction of crack growth for HCF-LCF mixed non-proportional loading of compressor blades using FRANC3D. The predictions correlated well with the experimental results and accounted for elastic and fracture resistance anisotropy and fracture mode asymmetry using FRANC3D software. Also, Witek [9] simulated the crack growth of a blade under resonant vibration due to bending through analytical methods and was able to successfully calibrate the final shape of the crack and the crack propagation life with experimental results.

Chapter 3 of this work will present a distinct numerical approach that was developed and used to combine steady and dynamic stresses as a basis of simulating 3D crack growth of components such as IBR compressor blades when subjected to HCF-LCF mixed non proportional loads. This work was recently published in Engineering Fracture Mechanics, titled under 'Modeling crack growth of an aircraft engine high pressure compressor blade under combined HCF and LCF loading' [12]. The method that was developed in [12] was used to successfully calibrate the direction, shape, and aspect ratio of the simulation with actual field components which fractured in service and can be extended on other IBRs and loading conditions.

For some aircraft engine components such as a blade/disk assemblies of bladed compressors, one of the controlling factors for life is fretting fatigue, commonly associated with HCF. Fretting is the relative tangential motion $\delta$ occurring between clamped bodies in contact when subjected to oscillatory forces [13]. Tangential amplitudes as 
low as $25 \mu \mathrm{m}$ to $100 \mu \mathrm{m}$ resulting from fretting can significantly reduce the fatigue performance of materials relative to their plain fatigue limits $[13,14]$. Fretting is generally accompanied with severe stress gradients localized at the edges of contact. Depending on, but not limited to other influential variables such as the COF, contact pressure, and loading, fretting may result in fretting fatigue, fretting wear, or a combination of both [15]. Fretting fatigue is distinct from fretting wear in that fatigue is the primary damage mechanism while wear is a secondary damage mechanism. Additionally, the nucleation life is controlled mostly by fretting whereas subsequent long crack propagation or crack arrest is controlled by stresses in the bulk of the material away from the contact region. This is in contrast to fretting wear, where very large strains and localized plastic conditions at edges of contact result in a permanent loss of material $[15,16,17]$. Further adding to this distinction, cracks nucleating from fretting fatigue typically grow normal to the contact surface and exist in elastic stress fields [13]. Thus, modeling techniques based on the fundamentals of LEFM have been previously used [8], [18] to successfully simulate crack growth for fretting fatigue applications.

Fretting fatigue is a costly problem associated with $\mathrm{HCF}$ and is a major life limiting factor for aircraft engine components, particularly for dovetail slot attachments used for blade-disk assemblies in compressors or turbines [14], [19]. The dovetail attachment fixture commonly used for bladed fans undergoes complex 3D non-proportional multiaxial stress states, where the three principal stresses change direction during loading [14]. These stress states are induced by steady and dynamic loading. Steady loads are generated mainly due to centrifugal forces acting on the dovetail interface at an applied angular velocity, with secondary steady loads arising due to thermal gradients which may occur during operation. Vibratory stresses are superimposed on steady stresses and are caused by aerodynamic or mechanical excitations acting on the blade which result in oscillatory motion at the blade-disk interface [19], [3], [20]. Several investigations were conducted to study and characterize the fretting fatigue behavior of aircraft engine components in service [20], [21], [22]. These investigations reveal that contrary to compressor or turbine blades where the influence of HCF is significant on both crack nucleation and crack growth [9], [10], [12], for fretting fatigue HCF is found to be a major contributor to crack nucleation whereas subsequent propagation is attributed mainly to LCF loading.

Damage caused by fretting is difficult to model and predict since both fatigue and wear occur concurrently with competing effects [16]. For this reason, earlier modeling attempts focused mainly on conditions where one of the damage mechanisms, namely fatigue, is dominant, while ignoring secondary damage considerations attributed to wear. As such, strain-based fatigue and critical plane methodologies such as the SWT model [23] have been successfully used in the past [24], [25], [26], [27] to determine fretting crack nucleation life as well as the location of the fretting nucleated crack for Ti-6Al-4V titanium alloys after taking into consideration the effects of contact [14]. The SWT criterion has also been used successfully [28] for predicting crack nucleation in contact subjected to fretting wear loading without the presence of bulk fatigue loads. Other critical plane fatigue models such as the Fatemi-Socie (FS) parameter [29] have also been used successfully to predict fretting fatigue [24]. However, it is thought that the SWT parameter is a more logical macroscopic model for fretting fatigue applications given that the localized tensile stresses dominate the characteristic initiation site [14].

Critical plane approaches although previously used successfully, lack a secondary damage mechanism attributed to fretting wear, and thus in some cases, could misrepresent the true fretting fatigue phenomenon. As early as 1984, 
Ruiz et al. [30] investigated the fatigue and fretting of a dovetail joint of a blade and disk, showing that cracks nucleated at locations associated with larger slip amplitudes. A fretting parameter was developed which considered effects of magnitude of slip $\delta$ in addition to the maximum tensile and shear stresses. The dependence of the overall fretting life on slip amplitude has also been demonstrated by Vingsbo et al. [31] using fretting maps, which show fretting life to decrease in the partial slip regime, and increase in the gross slip regime. In the gross slip regime, since wear is the primary damage mechanism, embryo cracks are worn away before having the opportunity to propagate to failure [17], [32]. Ding et al [33] expanded on this knowledge and developed a fretting specific fatigue parameter to characterize fretting damage by considering both fatigue and wear damage mechanisms. The developed modified SWT parameter is built on an understanding that fretting fatigue relies on the conjoint action of frictional work $\tau \delta$ which results in the nucleation of a crack near the edge of contact, and the SWT parameter which drives the growth of the crack. The damage parameter associated with wear includes a threshold limit which separates fretting fatigue from fretting wear, closely resembling observations made by Vingsbo et al. [31]. The modified SWT parameter was used on an aero-engine spline coupling application, showing accurate predictions of the fretting fatigue life and location of the cracks. However, it was concluded that the model requires more extensive validation.

Chapter 4 of this work will utilize the plain fatigue SWT parameter and the modified SWT parameter on an aircraft engine fan dovetail attachment made of a Ti-6Al-4V alloy. The effectiveness of these parameters in accurately predicting the nucleation location of the crack as well as the total cycles to nucleation will be studied. The chapter takes on a distinct approach to model the fretting fatigue behavior by utilizing a fracture mechanics based methodology to obtain a representative value for the COF between the dovetail fan blade and hub. By doing so, the evolution of COF due to an increasing number of cycles [24], [34] is considered in the analysis. This is an important contributor to the fretting fatigue behavior, but is often ignored in lifing methodologies [27]. For the dovetail fan attachment in question, the $\mathrm{COF}$ is minimum at initial assembly when the parts are undamaged with the presence of surface oxide films on the contact surfaces. When the oxide films are removed due to mechanical wear resulting from fretting, the underlying metals of the contact surfaces adhere, resulting in a strong increase to the COF. This work studies the capability for an 'average' value of COF to characterize the evolution of the COF in the nucleation and propagation phases of the fretting crack. This work has been recently published in the International Journal of Fatigue, titled under 'A fracture mechanics based approach for the fretting fatigue of aircraft engine fan dovetail attachments' [35]. 


\section{LITERATURE REVIEW}

\subsection{FUNDAMENTALS OF LEFM}

This chapter studies the fundamentals of LEFM and its application to compressor rotors of aircraft engines. The basic concepts and approaches of LEFM such as the energy approach and stress intensity approach will be studied, followed by a review of several closed form analytical solutions of the stress intensity factor (SIF) for simple loading and geometry. The crack tip plastic zone, crack closure, and threshold stress intensity phenomenon, and how they can be accounted for in various crack growth models will be explored. The accuracy of finite element approaches in the computation of stress intensity factors through the J-Integral and M-Integral method will also be studied. Various crack "kinking" phenomena for various loading and their correlation with experimental results will be considered.

From the studied literature, it is concluded that for accurate crack growth predictions, most recent works have shown that the Forman-Newman-de Koning (FNK) model employed by NASGRO V4 software best models crack growth as it accounts for the crack tip plastic zone, crack closure, and threshold stress intensity phenomenon. In addition to having an accurate crack growth model, one must also determine precise stress intensity factors for predicting crack growth behavior. The capability of crack growth software FRANC3D to accurately estimate stress intensity factors by use of path independent theories such as the J-Integral and M-Integral was studied. Furthermore, from the various crack-kinking models, it was determined that when mode I loading is dominant, the MTS is suitable for determining direction of crack growth. When there is significant contribution from other fracture modes, or when there is mixed mode non proportional loading, more accurate models must be employed.

\subsubsection{BACKGROUND \& HISTORY}

The study of fracture mechanics gained popularity with the vast increase of metals during the industrial revolution. Overtime, after several catastrophic failures, it was learnt that material defects in the form of pre-existing flaws could initiate cracks resulting in fractures at applied stresses much lower than yield. Most notably, the liberty ship failures during WWII, in which a total of 145 ships out of 2,500 had broken into two pieces due to a change in the manufacturing process where the ships were welded rather than riveted [36]. The ship failures occurred at locations which had low stresses. Investigations were carried out to reveal that the failures were a result of flaws, stress concentration factors, and brittle material properties due to low temperatures. The conventional way of design which primarily focused on tensile strength, yield strength, and buckling stress was inadequate in determining the material's capability in the presence of a crack. Since then, there has been significant growth and contribution of research to this recent scientific field and as a result fracture mechanics has become a powerful tool to design for high strength materials, primarily for civil and aerospace engineering applications.

The goal of fracture mechanics is to answer the question of what size of crack can be tolerated at service load, how long does it take for a crack to grow to a critical size, what size of pre-existing flaw can be permitted the moment the structure enters surface, and how often should inspection be carried out for cracks [36]. 


\subsubsection{ENERGY APPROACH}

Griffith (1921) was first to approach the crack problem using an energy balance formulation based on the first law of thermodynamics. Griffith determined that a crack will continue to propagate if the strain energy released upon crack growth is sufficient in providing the energy required to further break the surface bonds between the cracked surfaces. The energy release rate ' $G$ ' represents the amount of additional potential energy available for a unit area of crack extension, and is often referred to as the crack driving force. Griffith's approach will be used to derive the energy release rate of a cracked specimen with thickness 'B' and crack length 'a' shown below.

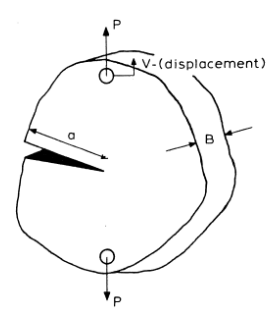

Fig. 4. Cracked specimen [36]

Conservation of energy can be applied to relate the total energy applied in the system due to an external force to the strain energy and energy released upon fracture. This can be represented in equation form as shown in (1), where ' $F$ ' is the total energy in the system due to work performed by an external force, (which in this case is 'P' in Fig. 4), ' $\mathrm{U}$ ' is the elastic energy stored in the system, and ' $\mathrm{W}$ ' is the work required to break bonds and create new surfaces:

$$
\mathrm{F}=\mathrm{U}+\mathrm{W}
$$

Griffith used the Inglis solution for an elliptical crack to show that upon loading, the strain energy in the system due to work done by an external force or applied nominal stress $\sigma$ is the following.

$$
U=U_{o}-\frac{\pi \sigma^{2} a^{2} B}{E}
$$

Due to conservation of energy, the energy release rate for an extension of unit area of crack surface ' $\mathrm{G}$ ' can be found assuming a unit thickness $(B=1)$, where ' $\Pi$ ' is the potential energy available for crack growth.

$$
\begin{gathered}
\mathrm{G}=-\frac{\mathrm{d} \Pi}{\mathrm{dA}}=\frac{\mathrm{d}}{\mathrm{dA}}(\mathrm{F}-\mathrm{U})=\frac{\mathrm{dW}}{\mathrm{dA}} \\
\mathrm{G}=\frac{\mathrm{d}}{\mathrm{dA}}\left(F-U_{o}+\frac{\pi \sigma^{2} \mathrm{a}^{2}}{\mathrm{E}}\right)=\frac{\mathrm{d}}{2 \mathrm{da}}\left(F-U_{o}+\frac{\pi \sigma^{2} \mathrm{a}^{2}}{\mathrm{E}}\right)=\frac{\pi \sigma^{2} \mathrm{a}}{\mathrm{E}}=\frac{\mathrm{dW}}{\mathrm{dA}}
\end{gathered}
$$

The energy required to generate two new crack surfaces is:

$$
\frac{\mathrm{dW}}{\mathrm{dA}}=2 \gamma_{\mathrm{s}}
$$

The stress at failure due to fracture is resolved to:

$$
\sigma_{\mathrm{f}}=\left[\frac{2 \mathrm{E} \gamma_{\mathrm{s}}}{\pi \mathrm{a}}\right]^{0.5}
$$

A modification was proposed by Orowan and Irwin to account for plastic deformation ahead of plastic zone. 


$$
\sigma_{\mathrm{f}}=\left[\frac{2 \mathrm{E}\left(\gamma_{\mathrm{s}}+\gamma_{\mathrm{p}}\right)}{\pi \mathrm{a}}\right]^{0.5}
$$

The stress intensity approach will be studied in depth in Section 2.1.3, however, it is mentioned here that one could calculate the stress intensity factor $\mathrm{K}$ for a known energy release rate $\mathrm{G}$ using the following equations:

$$
\begin{gathered}
\left.\mathrm{G}_{\mathrm{I}}=\frac{\mathrm{K}_{\mathrm{I}}^{2}}{\mathrm{E}} \text { (Plane Stress }\right)=\left(1-\mathrm{v}^{2}\right) \frac{\mathrm{K}_{\mathrm{I}}^{2}}{\mathrm{E}} \text { (Plane Strain) } \\
\mathrm{G}_{\mathrm{II}}=\left(1-\mathrm{v}^{2}\right) \frac{\mathrm{K}_{\mathrm{II}}^{2}}{\mathrm{E}} \\
\mathrm{G}_{\mathrm{III}}=(1+\mathrm{v}) \frac{\mathrm{K}_{\text {III }}^{2}}{\mathrm{E}}
\end{gathered}
$$

The total energy release rate can then be obtained by summing the energies from all modes. [36]

$$
\mathrm{G}=\mathrm{G}_{\mathrm{I}}+\mathrm{G}_{\mathrm{II}}+\mathrm{G}_{\mathrm{III}}
$$

\subsubsection{LOAD CONTROL VS DISPLACEMENT CONTROL}

The energy release rate, or crack driving force, can be derived for a load controlled or displacement-controlled specimen with a thickness 'B' as shown below.

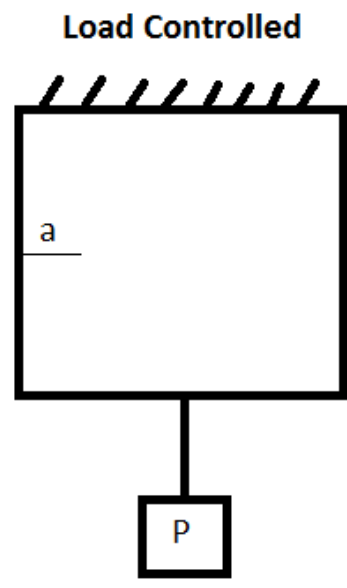

Displacement Controlled

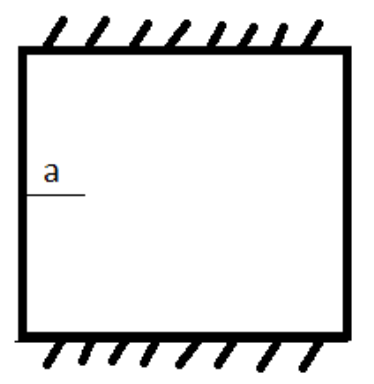

Fig. 5. Load controlled vs Displacement Controlled specimen

The general equation of the energy release rate (3) can be used to derive the energy release rate for a load controlled or displacement-controlled case.

$$
\begin{gathered}
\mathrm{G}_{\mathrm{P}=\text { const }}=-\frac{\mathrm{d} \Pi}{\mathrm{dA}}=\frac{\mathrm{d}}{\mathrm{dA}}(\mathrm{F}-\mathrm{U})=\frac{\mathrm{d}}{\mathrm{Bda}}\left(\mathrm{P} \Delta-\frac{\mathrm{P} \Delta}{2}\right)=\frac{\mathrm{P}}{2 \mathrm{~B}}\left(\frac{\mathrm{d} \Delta}{\mathrm{da}}\right) \\
\mathrm{G}_{\Delta=\text { const }}=-\frac{\mathrm{d} \Pi}{\mathrm{dA}}=\frac{\mathrm{d}}{\mathrm{dA}}(\mathrm{F}-\mathrm{U})=\frac{\mathrm{d}}{\mathrm{Bda}}\left(-\frac{\mathrm{P} \Delta}{2}\right)=-\frac{\Delta}{2 \mathrm{~B}}\left(\frac{\mathrm{dP}}{\mathrm{da}}\right)
\end{gathered}
$$


These equations can also be expressed through ' $c$ ', which is equal to compliance of the specimen. The compliance is better known as the inverse of the stiffness. Substituting this into equation (12) and (13):

$$
\begin{gathered}
c=\frac{\Delta}{\mathrm{P}} \\
\mathrm{G}_{\mathrm{P}=\text { const }}=-\mathrm{G}_{\Delta=\text { const }}=\frac{\mathrm{P}^{2}}{2 \mathrm{~B}} \frac{\mathrm{dc}}{\mathrm{da}}
\end{gathered}
$$

Equation (15) holds true since $\mathrm{dPd} \Delta$ is infinitesimal. Equation (15) is a very useful tool that can be used to obtain the strain energy release rate from experimental or measured data. One would calculate the compliance using the applied load and resulting displacement across varying crack lengths. The slope of the compliance can then be obtained with respect to crack size to calculate the energy release rate. The stress intensity factors can then be obtained through equations (8) to (11).

\subsubsection{CRITERIA FOR CRACK GROWTH}

Crack extension occurs when the energy release rate is equal to the energy required for crack growth. If the energy required for crack growth is constant and independent of crack length, one can conclude that once the energy release rate reaches this value, the specimen will fail.

However, materials have shown that depending on the length of the crack, the energy required to form a larger crack increases. This is illustrated below in the form of an R curve, which is a plot of the required energy to grow a crack per unit area surface extension with respect to crack length.

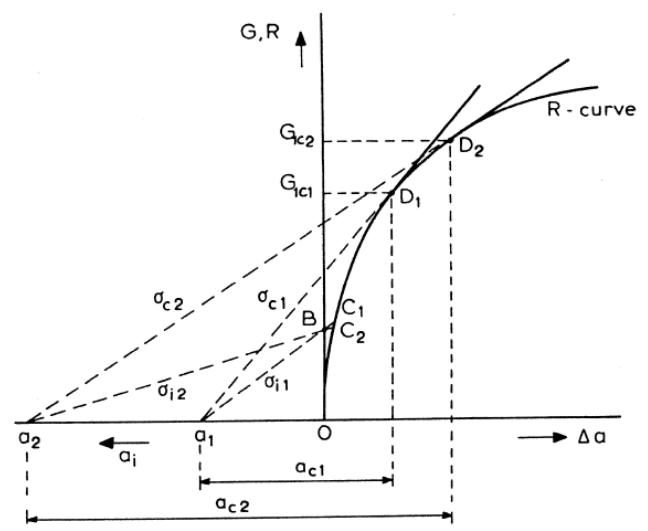

Fig. 6. R curve [36]

The solid dark line represents the materials ability to resist crack extension with respect to crack length. The dashed broken lines represent the applied energy release rate ' $G$ ' represented by equation (4), which is a function of applied stresses. For an applied stress of $\sigma_{1}$ at ' $\mathrm{C} 1$ ', the crack will propagate since the crack driving force is equal to the energy required to propagate the crack. Once propagating, the crack will enter a stable regime given that the energy required to form a larger crack is greater than the strain energy released. For an applied stress of $\sigma_{\mathrm{c} 1}$, the crack is always unstable as the crack driving force is always greater than the materials resistance to extend a crack. Thus, stable crack growth can be mathematically expressed through equations (16) and (17), where ' $R$ ' is the resistance curve for 
crack extension. The shape of the $\mathrm{R}$ curve is still a subject of discussion and the theory behind it is still not well yet established. The $\mathrm{R}$ curve depends on plate thickness and whether the loading can be approximated as plane strain (horizontal line) or plane stress.

$$
\begin{gathered}
\frac{d G}{d A} \leq \frac{d R}{d A} \\
G=R
\end{gathered}
$$

\subsubsection{STRESS INTENSITY APPROACH}

A perhaps more common approach in linear elastic fracture mechanics is to determine the stress field ahead of a crack for a particular geometry of interest. A crack in a solid can be stressed in three different modes illustrated below. Normal stress tends to open the crack in mode I, in plane shear slides the crack in mode II, and out of plane shear tears the crack in mode III.

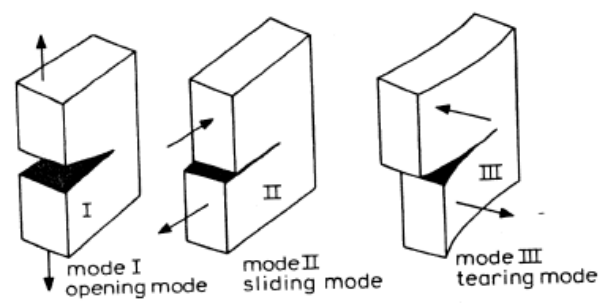

Fig. 7. Three modes of cracking [36]

For a through thickness crack of length 2a for an infinite plate loaded in Mode $\mathrm{I}$, the stresses in the vicinity of the crack tip can be computed through the stress intensity fields using equations (19) to (23). The ' $\theta$ ' and ' $r$ ' are the polar coordinates for angular and radial locations measured from the crack tip.

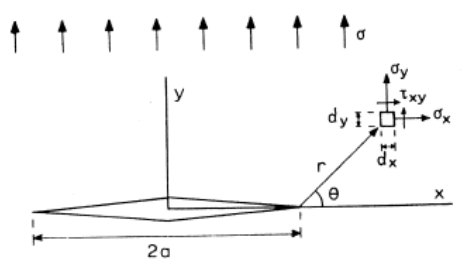

Fig. 8. Stress Intensity Fields for Mode I [36]

For Mode I loading, in a 'K-dominated' region, the stress field in any linear elastic isotropic cracked body can be expressed through (18), where $\mathrm{f}_{\mathrm{ij}}(\theta)$ is determined through complex stress functions depending on the geometry of the structure. In the equation, the leading term is dominant, and as such the stresses are proportional to $1 / \sqrt{\mathrm{r}}$ close to the crack tip in the singularity dominated zone. This holds true regardless of the configuration of the cracked body.

$$
\sigma_{i j}=\frac{K_{I}}{\sqrt{2 \pi r}} f_{i j}(\theta)+O\left(r^{0}\right)
$$


If the mode I, II, and III stress intensity factors are known, the complete stress state in the vicinity of the crack tip are known and can be computed using Table 1 for linear elastic isotropic materials. Equations (18) to (31) represent the stresses at the vicinity of a crack tip, at a distance ' $r$ ' and at an angle ' $\theta$ ' from the crack as shown in Fig. 8.

Table 1. Stress fields ahead of crack tip for Mode I, II, III

\begin{tabular}{|c|c|}
\hline \multicolumn{2}{|l|}{ Mode I } \\
\hline$\sigma_{x x}=\frac{K_{I}}{\sqrt{2 \pi r}} \cos \left(\frac{\theta}{2}\right)\left[1-\sin \left(\frac{\theta}{2}\right) \sin \left(\frac{3 \theta}{2}\right)\right]+O\left(r^{0}\right)$ & (19) \\
\hline$\sigma_{y y}=\frac{K_{I}}{\sqrt{2 \pi r}} \cos \left(\frac{\theta}{2}\right)\left[1+\sin \left(\frac{\theta}{2}\right) \sin \left(\frac{3 \theta}{2}\right)\right]+O\left(r^{0}\right)$ & $(20)$ \\
\hline$\sigma_{x y}=\frac{K_{I}}{\sqrt{2 \pi r}} \cos \left(\frac{\theta}{2}\right) \sin \left(\frac{\theta}{2}\right) \cos \left(\frac{3 \theta}{2}\right)+O\left(r^{0}\right)$ & $(21)$ \\
\hline$\sigma_{z z}=0($ Plane Stress $)=v\left(\sigma_{x x}+\sigma_{y y}\right)($ Plane strain $)$ & $(22)$ \\
\hline$\sigma_{x z}=\sigma_{y z}=0$ & $(23)$ \\
\hline \multicolumn{2}{|l|}{ Mode II } \\
\hline$\sigma_{x x}=-\frac{K_{I I}}{\sqrt{2 \pi r}} \sin \left(\frac{\theta}{2}\right)\left[2+\cos \left(\frac{\theta}{2}\right) \cos \left(\frac{3 \theta}{2}\right)\right]+O\left(r^{0}\right)$ & $(24)$ \\
\hline$\sigma_{y y}=\frac{K_{I I}}{\sqrt{2 \pi r}} \sin \left(\frac{\theta}{2}\right) \cos \left(\frac{\theta}{2}\right) \cos \left(\frac{3 \theta}{2}\right)++O\left(r^{0}\right)$ & $(25)$ \\
\hline$\sigma_{x y}=\frac{K_{I I}}{\sqrt{2 \pi r}} \cos \left(\frac{\theta}{2}\right)\left[1-\sin \left(\frac{\theta}{2}\right) \sin \left(\frac{3 \theta}{2}\right)\right]+O\left(r^{0}\right)$ & $(26)$ \\
\hline$\sigma_{z z}=0($ Plane Stress $)=v\left(\sigma_{x x}+\sigma_{y y}\right)($ Plane strain $)$ & $(27)$ \\
\hline$\sigma_{x z}=\sigma_{y z}=0$ & $(28)$ \\
\hline \multicolumn{2}{|l|}{ Mode III } \\
\hline$\sigma_{x x}=\sigma_{y y}=\sigma_{z z}=\sigma_{x y}=0$ & $(29)$ \\
\hline$\sigma_{x z}=-\frac{K_{I I I}}{\sqrt{2 \pi r}} \sin \left(\frac{\theta}{2}\right)$ & $(30)$ \\
\hline$\sigma_{y z}=\frac{K_{I I I}}{\sqrt{2 \pi r}} \cos \left(\frac{\theta}{2}\right)$ & $(31)$ \\
\hline
\end{tabular}




\subsubsection{ANALYTICAL CLOSED FORM SOLUTIONS OF STRESS INTENSITY FACTORS}

Stress intensity factors can be found through analytical methods, numerical methods, or through experimentation. This section will focus on the analytical methods used to determine the stress intensities for common crack configurations. First, an infinite sheet with evenly spaced collinear cracks is considered.
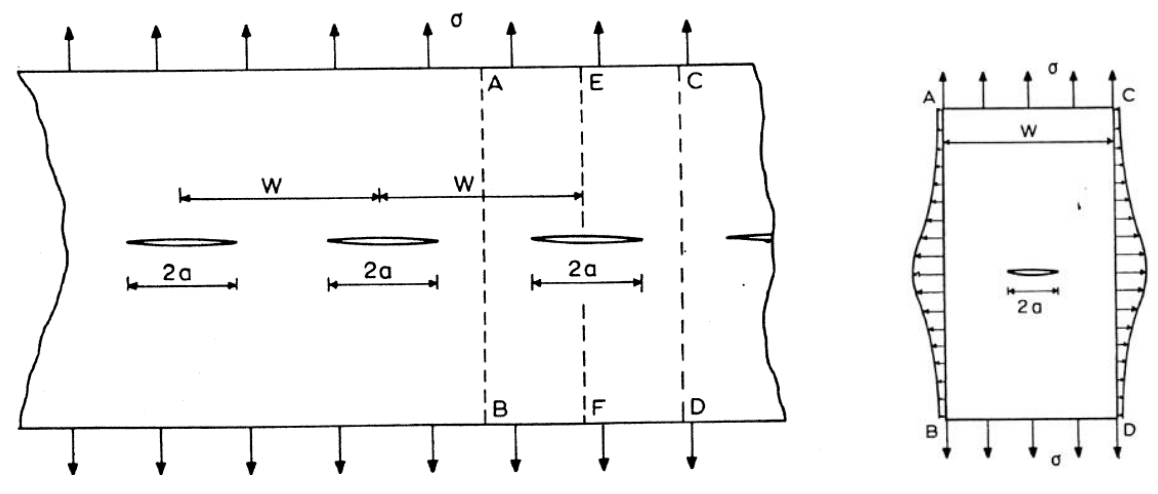

Fig. 9. Infinite plate [36]

The stress intensity factor is calculated by:

$$
K=\sigma \sqrt{\pi a}\left(\frac{W}{\pi A} \tan \left(\frac{\pi a}{W}\right)\right)^{0.5}
$$

By cutting the plate along $\mathrm{AB}$ and $\mathrm{CD}$, a strip of width $\mathrm{W}$ can be obtained. This strip contains shear forces which do not contribute much to K. Therefore, equation (32) can be approximated for a finite width strip. For very wide plates, or for configurations in which the crack length is very small compared to the width, the stress intensity reduces to only the first half, where the remaining square rooted portion approaches zero.

$$
K=\sigma \sqrt{\pi a}
$$

Usually, the stress intensity factors for finite width geometry are in the following form, where $\mathrm{Y}$ is a geometry factor which depends on the ratio of crack length to the width of the specimen.

$$
K=Y \sigma \sqrt{\pi a}
$$

For convenience, the form can also be presented be with respect to applied load.

$$
K_{I}=\frac{P}{B \sqrt{W}} f\left(\frac{a}{W}\right)
$$


Table 2. Geometry corrections for common test specimen [37]

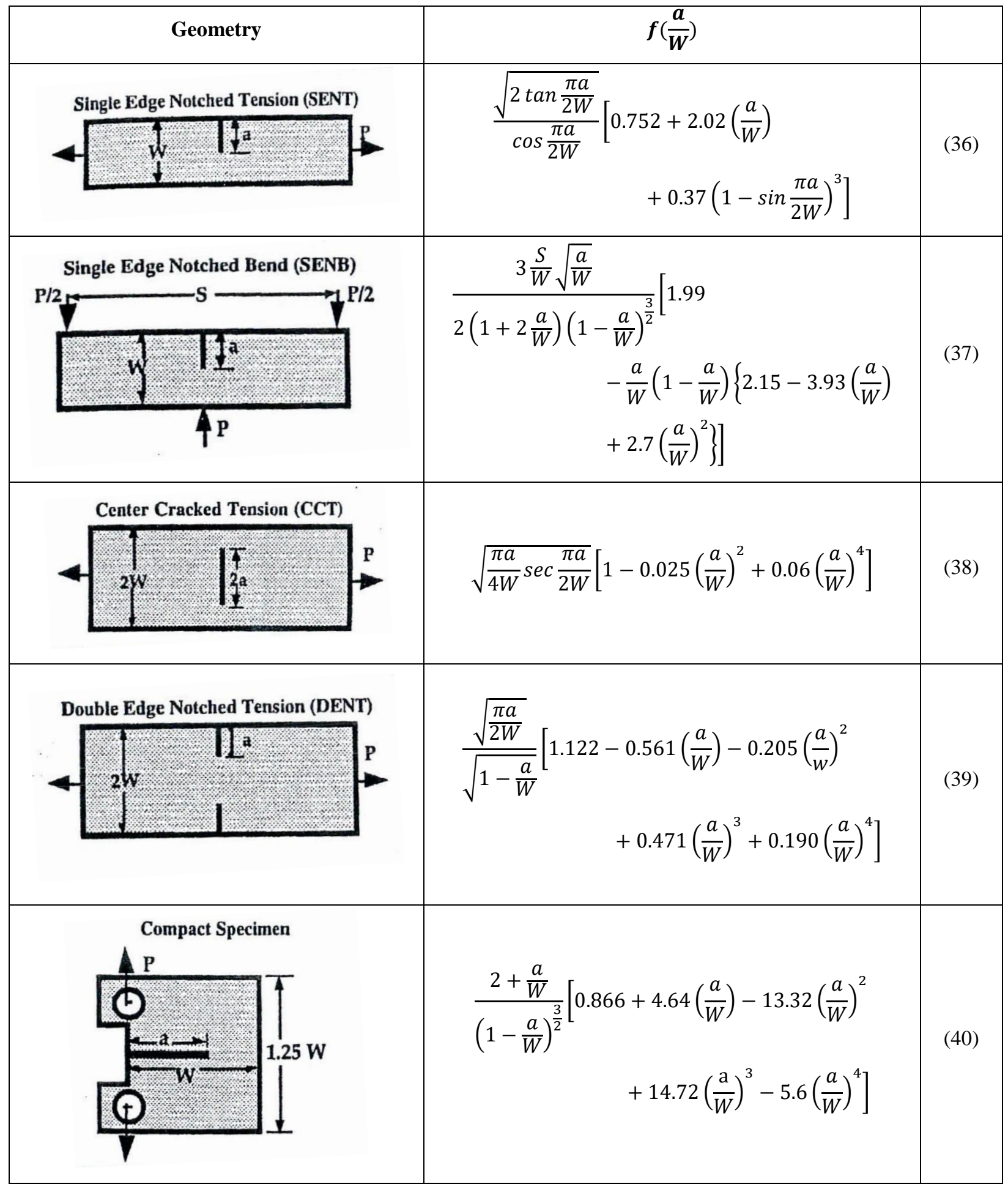




\subsection{ELLIPTICAL CRACKS}

Cracks often initiate at corners or edges and grow in quarter elliptical or semi elliptical shapes as shown below.

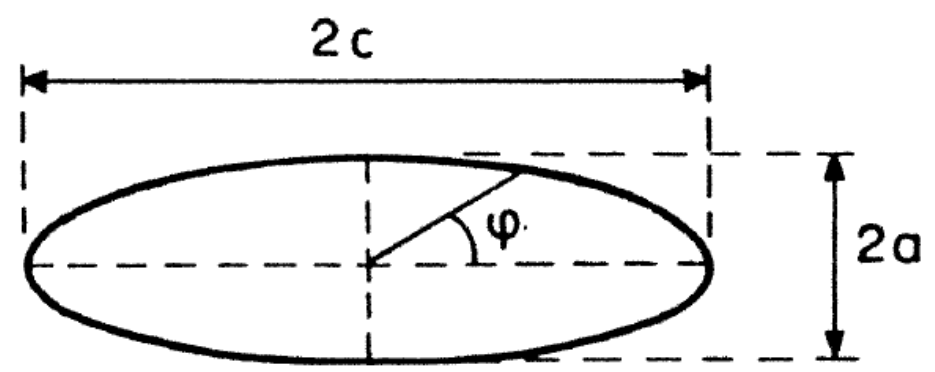

Fig. 10. Penny crack [36]

Sneddon was able to find the stress intensity factor in mode I as follows:

$$
\begin{gathered}
K_{I}=\frac{\sigma \sqrt{\pi a}}{\emptyset}\left(\sin ^{2} \Phi+\frac{a^{2}}{c^{2}} \cos ^{2} \Phi\right)^{1 / 4} \\
\varnothing=\frac{3 \pi}{8}+\frac{\pi a^{2}}{8 c^{2}}
\end{gathered}
$$

With slight modifications, equations (41) and (42) can be used for semi-elliptical flaws and quarter elliptical corner cracks. The equations show that KI varies along the crack front. When $\Phi=90$ degrees, the stress intensities are the largest, and when $\Phi=0$ degrees, the stress intensities are the lowest. The analytical solution for the stress intensity factor in mode I for a circular penny crack is obtained by setting $2 a=2 c$.

$$
K_{I}=2 \sigma \sqrt{\frac{a}{\pi}}
$$

To correct the surface flaw to an edge crack, a correction factor of 12 percent is applied to the stress intensity factor, and an effective crack length is used, which accounts for crack tip plasticity. Crack tip plasticity is explained in the proceeding sections.

$$
K_{I}=1.12 \frac{\sigma \sqrt{\pi a_{e f f}}}{\emptyset}\left(\sin ^{2} \Phi+\frac{a^{2}}{c^{2}} \cos ^{2} \Phi\right)^{1 / 4}
$$

\subsubsection{CRACK TIP PLASTICITY}

Equations (18) to (31) indicate that the stresses near a crack tip are directory proportional to applied loading (stress intensity) and inversely proportional to the square of radius (distance from crack tip). From the analytical form alone, one can deduce that at a distance very close to the crack tip, there is a singularity problem in which the stresses reach infinity. However, materials prohibit stresses becoming infinite at a crack tip. Rather, plastic deformation results in a plastic zone with a stress level equal to that of yield. 


\subsection{IRWIN PLASTIC ZONE CORRECTION}

A plastic zone of radius $r_{p}^{*}$ can be approximated such that the material within the plastic zone experiences a yield stress. By using the stress intensity fields at the crack tip in the "Y" direction, the distance away from the crack tip can be found in which the computed stress is equal to the yield stress of the material. In other words, $r_{p}^{*}$ can be found by setting $(\theta=0)$ directly ahead of the crack and $\left(\sigma_{y y}=\sigma_{y s}\right)$ in equation (20).
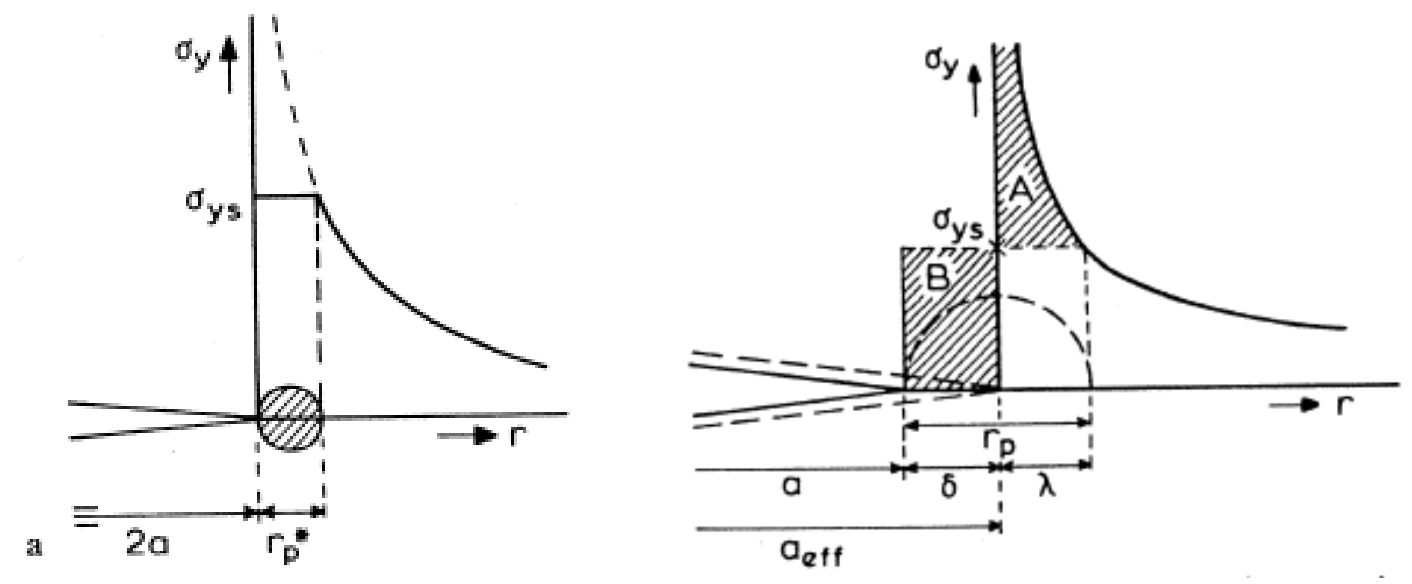

Fig. 11. Irwin plastic zone correction [36]

From the figure on the left-hand side, one can calculate the following:

$$
\begin{gathered}
\sigma_{y y}=\sigma_{y s}=\frac{K_{I}}{\sqrt{2 \pi r_{p}^{*}}} \\
r_{p}^{*}=\frac{K_{I}^{2}}{2 \pi \sigma_{y s}^{2}}
\end{gathered}
$$

The plastic zone is somewhat larger as stresses above yielding (from 0 to $r_{p}^{*}$ ) represented by shaded area must be accounted for and redistributed directly ahead of the crack tip. Therefore, area A must equal area B for the figure on the right-hand side. Irwin argued that the presence of plasticity makes the crack behave as if it were larger in size. The effective crack length therefore must be found which is $a+\delta$. After integrating and setting the areas to equal, the effective crack area can be calculated through:

$$
\begin{aligned}
r_{p} & =2 r_{p}^{*} \\
a_{e f f} & =a+r_{p}^{*}
\end{aligned}
$$

Cracks which are loaded to the same Mode I stress intensity have plastic zones of equal size according to equation (46). Furthermore, outside the plastic zones, the stress field will be the same. 


\subsection{DUGDALE STRIP YIELD MODEL}

The Dugdale approach also determines an effective crack length which is longer than the physical crack through a strip yield model shown below. [38]
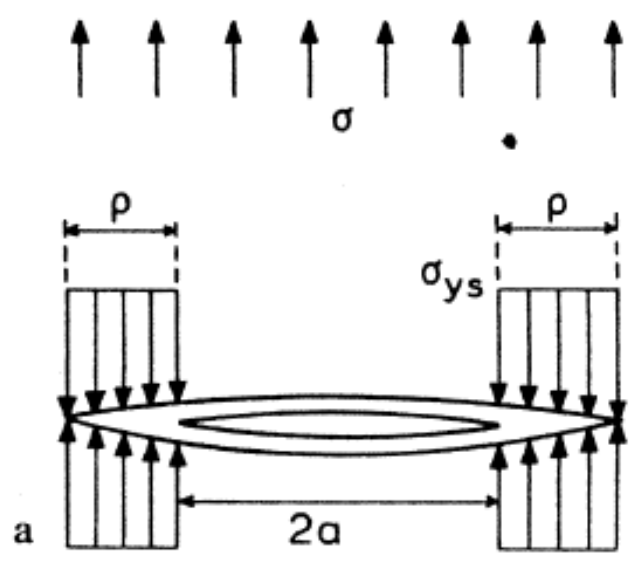

\section{b}

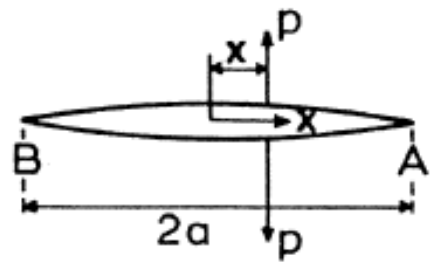

Fig. 12. Strip yield model [36]

In the strip yield model, it is assumed that the crack edges in front of the physical crack carry yield stress which tends to close the crack. However, the region indicated by $\rho$ is not truly cracked as the material holds the yield stress. The size of this region is chosen with the intention of obtaining a stress intensity of zero, which would negate the singularity issue. Therefore, the stress intensity $K_{\sigma}$ due to the uniform stress must be compensated by the stress intensity $K_{P}$ due to the wedge forces which have a magnitude of yield stress. The stress intensity due to wedge forces 'P' can be calculated through the following equations:

$$
\begin{gathered}
K_{A, B}=\frac{P}{\sqrt{\pi a}} \sqrt{\frac{a \pm x}{a \bar{\mp}}} \\
K=\frac{P}{\sqrt{\pi a}} \int_{s}^{a}\left[\sqrt{\frac{a+x}{a-x}}+\sqrt{\frac{a-x}{a+x}}\right] d x=2 P \sqrt{\frac{a}{\pi}} \arccos \left(\frac{s}{a}\right)
\end{gathered}
$$

The integral must be taken from $a$ to $a+\rho$ and $P=\sigma_{y s}$. The $\rho$ can be found by neglecting higher order terms.

$$
\rho=\frac{\pi K_{I}^{2}}{8 \sigma_{y s}^{2}}=r_{p}^{*}
$$

The Dugdale approach and Irwin approach for plasticity corrections are almost identical. 


\subsection{PLASTIC ZONE SHAPE}

The shape of the plastic zone can be determined through use of a yield criteria. The Von-Mises criterion will be considered. The principal stresses can be calculated using plane stress conditions through mode I stress intensity fields.

$$
\begin{gathered}
\left(\sigma_{1}-\sigma_{2}\right)^{2}+\left(\sigma_{2}-\sigma_{3}\right)^{2}+\left(\sigma_{3}-\sigma_{1}\right)^{2}=2 \sigma_{y s}^{2} \\
\sigma_{1,2}=\frac{\sigma_{x x}+\sigma_{y y}}{2} \pm \sqrt{\left(\frac{\sigma_{x x}-\sigma_{y y}}{2}\right)^{2}+\sigma_{x y}^{2}} \\
\sigma_{1,2}=\frac{K}{\sqrt{2 \pi r}} \cos \left(\frac{\theta}{2}\right)\left(1 \pm \sin \left(\frac{\theta}{2}\right)\right) \\
\sigma_{3}=v\left(\sigma_{1}+\sigma_{2}\right)=2 v \frac{K}{\sqrt{2 \pi r}} \cos \left(\frac{\theta}{2}\right) \text { (Plane Strain) } \sigma_{3}=0 \text { (Plane Stress) }
\end{gathered}
$$

The plastic zone boundary can then be computed as a function of polar coordinate $\theta$ by substituting equations (53) through (55) to equation (52). The resulting plastic zone radii are:

$$
\begin{gathered}
\left.r_{p}(\theta)=\frac{K^{2}}{4 \pi \sigma_{y s}^{2}}\left[\frac{3}{2} \sin ^{2} \theta+(1-2 v)^{2}(1+\cos \theta)\right]\right] \text { (Plane Strain) } \\
\left.r_{p}(\theta)=\frac{K^{2}}{4 \pi \sigma_{y s}^{2}}\left[1+\frac{3}{2} \sin ^{2} \theta+\cos \theta\right]\right] \text { (Plane Stress) }
\end{gathered}
$$

The resulting shape can be plotted as shown below.
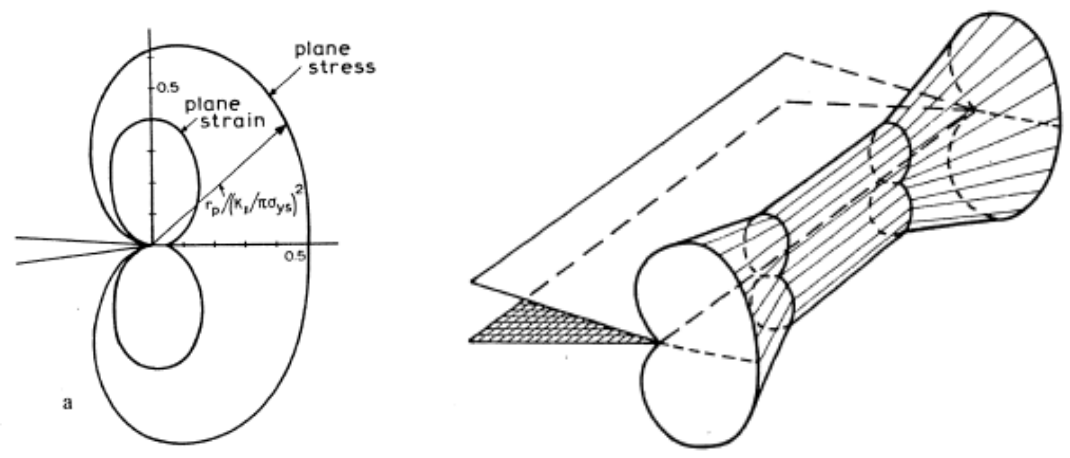

Fig. 13. Plastic zone shape [36]

The plane strain plastic zone is smaller in size compared to the plane stress plastic zone. This is because for a plane strain condition, the material is more restricted from expanding due to additional presence of material surrounding it from its sides. However, the plane stress condition (at the free surface) does not have material restricting the plastic zone from expanding, resulting in further plasticity. Other factors such as the strain hardening phenomenon and the level of applied stress can affect the shape. 


\subsubsection{CRACK GROWTH MODELS}

Crack growth models are used to relate the applied stress intensity range to the crack growth rate. These models are formulated through the curve fitting empirical data. Initially, crack growth models were estimated through simple power function which only depended on the applied stress intensity range. Since then, there has been significant improvement in estimating crack growth rates by considering other factors such as threshold stress intensity, crack tip plasticity, and crack retardation. This section will consider how these phenomena can be modelled. Below illustrates a classical crack growth rate relationship with respect to stress intensity range.

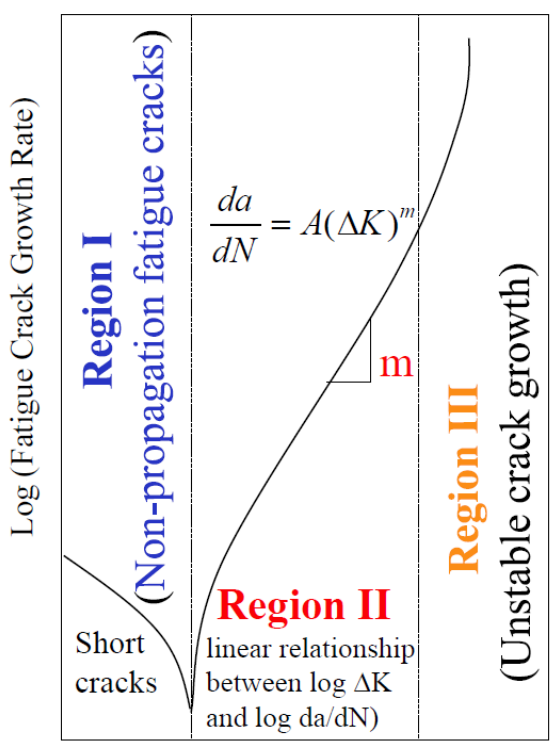

Fig. 14. Typical crack growth model [39]

There are three regions of crack growth. Region I is associated to small cracks and is characterized by nonpropagating fatigue cracks due to microstructural barriers caused by grain boundaries. A short crack is a physical discontinuity in a material which exhibits crack growth behavior which is not characterized by LEFM. The lower limit of a short crack is considered the length at initiation and the upper limit is the length in which LEFM starts to accurately describe the crack behavior [40]. Newman characterized a short crack to be $10 \mu \mathrm{m}$ to $1 \mathrm{~mm}$ in size [41]

Crack initiation in alloys results from formation of intrusions, extrusions and persistent slip bands followed by crack extension in shear mode at free surface. This is stage I crack growth, also referred to as an incipient crack. After nucleation of a crack, the crack propagates at a high rate and is sensitive to crystallographic conditions.

Following stage I crack growth is stage II, which is the extension of the crack in a direction perpendicular to maximum applied stress. This is one of the several crack extension models which will be studied in this literature review in Section 2.1.6. Stage II is also referred to as the hesitating crack which is when the crack enters a long stage of slow growth for 10 to 70 percent of total life. The third and final stage is the running crack, where the growth rate becomes insensitive to crystallographic properties and is increased again. [40] 
Paris, Gomez, and Anderson were the first to assume a simple power function between the applied stress intensity range and the crack growth rate.

$$
\frac{d a}{d N}=C \Delta K^{n}
$$

Further works showed that the crack growth rate also depends on the loading ratio such that [36]:

$$
\frac{d a}{d N}=f(\Delta K, R)
$$

It has also been proposed that the crack growth is related to the maximum applied stress intensity through the following [36]:

$$
\frac{d a}{d N}=C K_{\max } \Delta K^{n}
$$

These crack growth models predict well the growth rate in region II of Fig. 14, and exhibit a linear relationship when plotted in logarithmic scale. Subsequent studies attempted to account for region I and region III by considering the effects of crack closure and threshold stress intensity on crack growth. These phenomena and how to modify the crack growth model to account for them will be explored in the proceeding sections.

\subsubsection{CRACK CLOSURE}

Fatigue crack growth is difficult to model due to two issues, one being the measurement of the fatigue threshold and the other is the stress intensity factor which opens the crack [42]. Crack opening could also be thought of as the transition from cyclic compressive strains to tensile strains near the crack front.

Elber was first to introduce the concept of crack closure [43]. Crack closure contributions are significant and are responsible for crack retardation effects. Crack closure can arise from various phenomena such as asperities which refer to roughness induced closure, corrosion induced closure, and plasticity induced closure. Several finite element models have been employed to show that compressive stresses arise from the plastic zone near the crack tip which tends to close the crack. Furthermore, it was shown that that the closure is more pronounced in plane stress conditions than in plane strain due to an enhanced plasticity effect. When overload occurs, additional plastic flow is introduced to the crack tip, resulting in a crack arrest. It is important to model the crack closure effect due to plasticity using a closure model.

Elber also argued that crack closure can arise even when loading in tension whereas before it was thought to only occur in compression. When a crack is advanced, there is permanent tensile plastic deformation in the wake of a crack which creates partial crack closure. Elber concluded that crack closure is a consequence of tensile plastic deformation left in the wake of a propagating crack. Therefore, a crack is fully open for only part of the load cycle even when the loading is fully in tension. This is important to consider when modelling the crack growth of a compressor disk, which is under tension due to centripetal forces. To model the crack closure effectivity, one must determine the relation between the crack opening and closing load [43].

Several experiments on metallic materials under aircraft spectrum loading have validated Elber's hypothesis to show that fatigue cracks remain closed during part of the load cycle under constant and variable amplitude tension 
loading. Therefore, it is important to incorporate the crack closure concept to accurately correlate crack growth rates under constant amplitude loading. Newman has formulated a crack growth model which considers the residual plastic deformation in the wake of an advancing crack. The crack tip opening displacements are used to calculate the contact stresses during unloading which are influenced by plastic yielding at the tip of a crack. When reloading, the applied stress at which the crack becomes open is related to these contact stresses. This level of stress is called the crack opening stress. Therefore, one must first subtract the opening stress intensity range from the applied stress intensity range to result in an effective stress intensity which works to grow the crack [44].

\subsubsection{THRESHOLD STRESS INTENSITY}

The fatigue threshold is the stress intensity range in which no crack growth occurs. Several works employ one million cycles with no crack extension as a reliable $\Delta K_{t h}$ value. The threshold stress intensity has a strong dependence to the loading ratio. For example, tests show that for $R=0$, that the threshold is twice as much the value as it is for load ratios larger than 0.5 .

Practically, one can explain this phenomenon by considering the nature of crack closure. When a specimen undergoes fatigue loading with lower mean stress, crack closure has a more prominent effect as the loading is closer to compression verses fatigue loading at higher mean stress. Therefore, as mean stress is lower, there is larger amplitude of loading required to grow the crack at a detectable limit.

The R-dependence of $\Delta K_{t h}$ is not a material constant. Schmidt and Paris proposed to subtract from $\Delta K_{t h}$ the stress intensity range required to open the crack $K_{\text {open }}$. The fatigue tolerance range $\Delta K^{T}$ is a material constant which represents the additional stress intensity required starting from an open crack, up to the threshold. This can be represented by: [45]

$$
\Delta K_{T}=\Delta K_{t h}-\left(K_{\text {open }}-K_{\text {min }}\right)
$$

Threshold stress intensities can be obtained for various loading ratio for alloys and there exists extensive databases for fatigue propagation thresholds in alloys obtained through experimental work. However, stress intensity thresholds lose meaning in the Region I small crack regime. Microstructural cracks can grow at a higher rate than corresponding large cracks (Region II) at the same applied force and can propagate at stress intensity levels below the large crack threshold. Therefore, design against HCF failure must be based on a notion of a small crack threshold under high frequency. There are several reasons as to why small cracks behave differently from large cracks. The microstructure leads to accelerated crack advance along weak paths which provide microstructure features oriented for easy crack growth. Furthermore, for very small cracks, the local plasticity ahead of the crack tip where the assumption of a small scale yielding in the use of the SIF is not valid, making LEFM inapplicable. Also, the reduced role of crack tip shielding for small cracks due to crack closure results in a larger stress intensity range than the corresponding large crack at the same K levels. Small cracks therefore grow below the large crack threshold at velocities greater than equivalently large cracks. [10]

The relation between the load ratio on the fatigue threshold in metals, specifically for Ti-6Al-4V, a popular choice of metal for compressor disks has been studied by Boyce and Ritchie. Their goal was to examine the effects of 
loading frequencies and loading ratios on crack propagation. They found that by increasing the load ratio, the threshold stress intensity range decreases. Schmidt and Paris explain this behavior because of the crack closure concept. Regards to loading frequencies, they found that by varying the loading frequencies from $50 \mathrm{~Hz}$ to $1000 \mathrm{~Hz}$, there is no change in the threshold stress intensity range. Therefore, it was concluded that the threshold stress intensity range is independent of frequency of loading and rather, it is the role of the load ratio at near threshold levels. For low load ratios, the closure-based crack propagation model describes the threshold behavior reasonably well. In the case of for Ti-6Al-4V, Boyce and Ritchie found that even for R ratios ranging from 0.5 to 0.95 , there still exists a significant reduction in the threshold stress intensity range. [46]

\subsubsection{FNK MODEL}

The FNK model was formulated which accounts for the retardation near threshold, acceleration near fast fracture and crack closure effects. The $C, n, p$, and, $q$ are empirical constants derived by curve fitting test data at varying environmental conditions such as temperature. $\Delta K$ is the applied stress intensity factor range, $\Delta K_{t h}$ is the threshold stress intensity range which is also dependent on loading ratio, and $K_{c}$ is the critical stress intensity factor. This same model is used by the NASGRO software to predict fatigue crack growth in various metals for constant and variable amplitude loading. [47]

$$
\frac{d a}{d N}=\frac{C(1-f)^{n} \Delta K^{n}\left(1-\frac{\Delta K_{t h}}{\Delta K}\right)^{p}}{(1-R)^{n}\left(1-\frac{\Delta K}{(1-R) K_{c}}\right)^{q}}
$$

The $f$ function can be expressing according to Newman's crack closure phenomenon as: [44]

$$
\begin{gathered}
f=\left\{\begin{array}{l}
\max \left(R, A_{o}+A_{1} R+A_{2} R^{2}+A_{3} R^{3}\right. \\
-2 \leq R<0 \\
A_{o}+A_{1} R
\end{array}\right. \\
A_{o}=\left(0.825-0.34 \alpha+0.05 \alpha^{2}\right)\left[\cos \left(\frac{\frac{\pi}{2} S_{\text {max }}}{\sigma_{o}}\right)\right]^{\frac{1}{\alpha}} \\
A_{1}=(0.415-0.071 \alpha) * \frac{S_{\text {max }}}{\sigma_{o}} \\
A_{2}=1-A_{o}-A_{1}-A_{3} \\
A_{3}=2 A_{o}+A_{1}-1
\end{gathered}
$$

The threshold stress intensities can be calculated for varying R- ratios as follows: [48]

$$
\Delta K_{t h}= \begin{cases}\frac{\Delta K\left[\frac{1-R}{1-f}\right]^{1+R C_{t h}^{p}}}{\left(1-A_{o}\right)^{(1-R) C_{t h}^{p}}} & R \geq 0 \\ \frac{\Delta \mathrm{K}\left[\frac{1-R}{1-f}\right]^{1+R C_{t h}^{m}}}{\left(1-A_{o}\right)^{\left(c_{t h}^{p}-R C_{t h}^{m}\right)}} & R<0\end{cases}
$$




\subsubsection{BRIEF REVIEW OF ELASTIC PLASTIC FRACTURE MECHANICS}

For small scale crack tip plasticity, the energy release rate is not affected by the plastic deformation at the crack tip. However, for larger plastic zones, its influence on the energy release rate is pronounced. This chapter gives a very brief introduction to the J-integral and M-integral methods which can be employed to obtain the stress intensity factors when plasticity effects are large. Using the displacements along the crack front of a 3D finite model, the $\mathrm{J}$ integral or M integral can be numerically calculated and then converted into an equivalent stress intensity factor. Generally, the M-integral gives more accurate results than the J-integral.

\subsubsection{J-INTEGRAL}

The J-Integral, formulated by James Rice establishes an elastic-plastic solution of the crack tip stress field. The J-Integral is defined by integrating counterclockwise surrounding an area of a stressed solid. $T$ is the tension vector perpendicular to the integrated path, $u$ is the displacement in the $\mathrm{x}$ direction and $d s$ is an element along a path which closes the contour $\Gamma$. $W$ is the strain energy per unit volume. Rice was able to show that for a closed contour, the integral defined by equation (69) is equal to zero. [49]

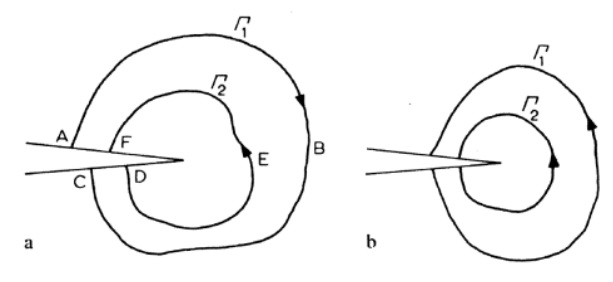

$$
\begin{gathered}
J=\int_{\Gamma}\left(W d y-T \frac{\partial u}{\partial x} d s\right) \\
W=W(x, y)=\int_{0}^{\varepsilon} \sigma_{i j} d \epsilon_{i j}=\frac{1}{2} \sigma_{i j} \epsilon_{i j}
\end{gathered}
$$

Fig. 15. Definition of J Integral [36]

Rice also applied the integral to crack problems. For the crack tip shown in Fig. 15, the J integral evaluated for the closed contour $\mathrm{ABCDEF}$ is zero. However, since the tension vector and $d y$ are equal to zero along $\mathrm{AF}$ and $\mathrm{CD}$, the contribution of these parts is zero. Therefore, the contribution of $\mathrm{ABC}$ is equal in magnitude to the contribution of DEF, thus the integral is path independent. However, it should be noted that the integral $J_{\Gamma 1}$ is not taken along a closed contour, but rather ends at the crack edges.

For an elastic stress field solution, the $\mathbf{J}$ integral results in the familiar energy release rate $\mathrm{G}$. Therefore, the $\mathbf{J}$ integral is a generalized energy release rate due to crack propagation which is also valid when there is crack tip plasticity. Since the J-integral is path independent, the easiest path can be selected for the integration, for example, at the edges of the specimen. The $\mathrm{J}$ integral, like the energy release rate $\mathrm{G}$ can be converted into stress intensity factors using equations (8) to (11).

\subsubsection{M-INTEGRAL}

Yau et.al, developed the M-integral method which was built off the J-integral to extract the stress intensity factors for the three modes from the global energy release rate. [50]

$$
M^{1,2}=\int\left(\sigma_{i j}^{(1)} \frac{\partial u_{i}^{(2)}}{\partial x_{1}}+\sigma_{i j}^{(2)} \frac{\partial u_{i}^{(1)}}{\partial x_{1}}-W^{(1,2)} \delta_{1 j}\right) \frac{\partial q}{\partial x_{j}} d V \quad i=1,2,3 j=1,2
$$




\subsubsection{CRACK KINK PHENOMENON}

This chapter studies the various models which could be employed to estimate the direction of a crack, including energy-based theories.

\subsubsection{MIX MODE STRESS INTENSITY FACTORS}

When the plane of loading is not perpendicular to the crack, the applied load can be resolved into its basic components. By doing so, two or more modes of crack growth are introduced to the model. The contribution of all applied modes to the stresses at the vicinity of the crack must be summated using superposition. Consider the following loading configuration.

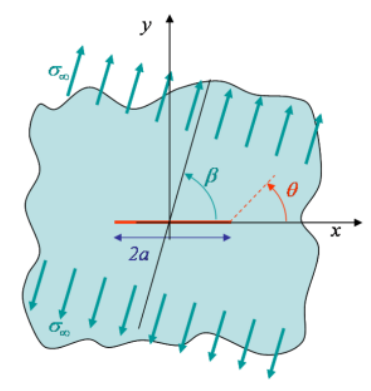

Fig. 16. Mixed mode loading [51]

Using superposition principle and omitting higher order terms which represent far field stresses, the stress field for mixed mode I and mode II loading near crack tip results in:

$$
\begin{gathered}
\sum \sigma_{x x}=\frac{1}{\sqrt{2 \pi r}}\left[K_{I} \cos \left(\frac{\theta}{2}\right)-\frac{K_{I}}{2} \sin \left(\frac{\theta}{2}\right) \sin \left(\frac{3 \theta}{2}\right)-2 K_{I I} \sin \left(\frac{\theta}{2}\right)-\frac{K_{I I}}{2} \sin \left(\frac{\theta}{2}\right) \cos \left(\frac{3 \theta}{2}\right)\right] \\
\sum \sigma_{y y}=\frac{1}{\sqrt{2 \pi r}}\left[K_{I} \cos \left(\frac{\theta}{2}\right)+\frac{K_{I}}{2} \sin \left(\frac{\theta}{2}\right) \sin \left(\frac{3 \theta}{2}\right)+\frac{K_{I I}}{2} \sin \left(\frac{\theta}{2}\right) \cos \left(\frac{3 \theta}{2}\right)\right] \\
\sum \sigma_{x y}=\frac{1}{\sqrt{2 \pi r}}\left[\frac{K_{I}}{2} \sin \left(\frac{\theta}{2}\right) \cos \left(\frac{3 \theta}{2}\right)+K_{I I} \cos \left(\frac{\theta}{2}\right)-\frac{K_{I I}}{2} \sin \left(\frac{\theta}{2}\right) \sin \left(\frac{3 \theta}{2}\right)\right]
\end{gathered}
$$

The stresses and corresponding stress intensity factors can be resolved into polar coordinates.

$$
\begin{gathered}
\sigma_{\theta \theta}(\theta=\beta)=\sigma_{y y} \cos ^{2} \beta+\sigma_{x x} \sin ^{2} \beta-2 \sigma_{x y} \sin \beta \cos \beta \\
\sigma_{\theta \theta}=\frac{1}{\sqrt{2 \pi r}} \cos \left(\frac{\theta}{2}\right)\left[K_{I} \cos ^{2}\left(\frac{\theta}{2}\right)-\frac{3}{2} K_{I I} \sin \theta\right] \\
K_{I}(\Delta \theta)=\sigma_{\theta \theta} \sqrt{2 \pi r}=\cos \frac{\Delta \theta}{2}\left[K_{I} \cos ^{2} \frac{\Delta \theta}{2}-\frac{3}{2} K_{I I} \sin \Delta \theta\right] \\
K_{I I}(\Delta \theta)=\sigma_{r \theta} \sqrt{2 \pi r}=\frac{1}{2} \cos \frac{\Delta \theta}{2}\left[K_{I} \sin \Delta \theta+K_{I I}(3 \cos \Delta \theta-1)\right] \\
K_{I I I}(\Delta \theta)=\sigma_{z \theta} \sqrt{2 \pi r}=K_{I I I} \cos \frac{\Delta \theta}{2}
\end{gathered}
$$




\subsubsection{MAX TENSILE STRESS (MTS)}

The MTS predicts that the direction in which a crack will propagate is dependent on the angle $\theta$ that results in the maximum hoop stress represented by equation (76). To maximize the tensile stress in the $\theta$ direction, equation (77) which represents stress intensity factor in the hoop direction can be differentiated with respect to $\theta$ and set equal to zero. Note that maximizing the hoop stress is equivalent to maximizing the stress intensity factor in the hoop direction. This results in the following kink angle. [51]

$$
\Delta \theta_{c}=2 \tan ^{-1}\left(\frac{1-\sqrt{1+8\left(\frac{K_{I I}}{K_{I}}\right)^{2}}}{4\left(\frac{K_{I I}}{K_{I}}\right)}\right)
$$

For proportional loading, the ratio between mode I and II stress intensity factor remain constant. According to equation (80), for a constant $K_{I I} / K_{I}$, the resulting kink angle $\Delta \theta_{c}$ would be constant. One could also conclude that maximizing the tensile stress given by equation (76) can be done by growing in a path which $K_{I I}=0$. In pure mode I loading $\left(K_{I I}=0\right)$, the kink angle becomes perpendicular to the applied load ( $\Delta \theta_{c}=90$ degrees). For pure mode II loading $\left(K_{I}=0\right)$, the maximum hoop stress occurs when $\Delta \theta_{c}=70.5$ degrees.

\subsubsection{MAX SHEAR STRESS (MSS)}

Experimental data obtained from work done by Erdogan, Sih, Maiti, and Smith show that the assumption that a crack propagates only by a mode I mechanism is valid only for brittle materials. In mixed mode loading of steels and aluminum alloys, Otsuka and Gao Hua produced mode II cracks.

Otsuka's approach was to assume a crack either grows as mode I (perpendicular to MTS), or mode II (plane of maximum shear stress - MSS). Melin used a similar approach by comparing the mode I and II stress intensity factors to the fracture toughness in mode I and mode II. If the fracture toughness in both modes is known, one could determine the mode of crack growth and the critical load for fracture under mixed mode loading. The maximum shear stress theory predicts that a crack will propagate in a direction in which the resolved shear stress due to mode II and mode III is the maximum.

$$
\sigma_{s}=\sqrt{\sigma_{r \theta}^{2}+\sigma_{z \theta}^{2}} \propto K_{s}=\sqrt{K_{I I}(\Delta \theta)^{2}+K_{I I I}(\Delta \theta)^{2}}
$$

Like MTS, the angle at which failure occurs due to MSS can be calculated by maximizing the resolved shear stress, $K_{S}$ in equation (81) and differentiating with respect to $\theta$ and finding $\theta$ which results in zero. For pure mode II loading, a crack propagating due to MSS theory would grow coplanar. The proceeding figure shows how cracks will propagate through MTS \& MSS under both pure mode II loading (b), and mix mode loading (a). 
(a)

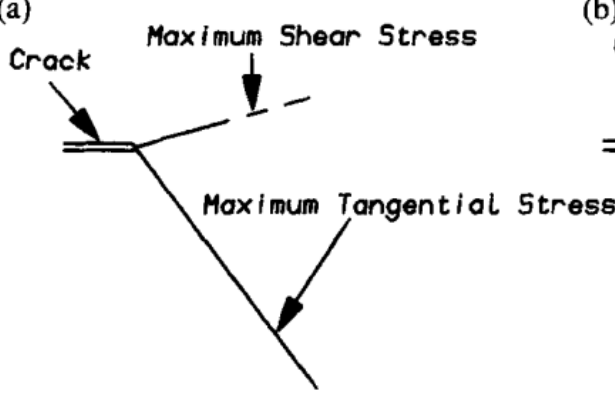

(b)

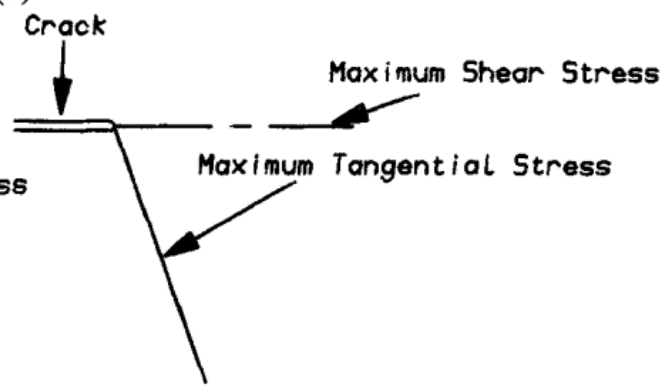

Fig. 17. MTS \& MSS for a) KI/KII not zero and b) KI/KII=0 [51]

To determine whether a crack will grow under mode I or mode II, one must conduct experimental studies. Studies of proportional loading of stainless steels show that when loading is dominated by mode II, the MSS criterion is reliable, and similarly when loading is dominated by mode I, the MTS is reliable.

Chao and Liu [52] obtained the sharp transition of MTS crack growth to MSS crack growth using the hoop and shear stress allowable of the material. By determining the ratio $K_{I I} / K_{I}$ at location ' $\mathrm{C}$ ' in the figure below, one can predict when a crack will change its directional nature to align itself according to the MTS or MSS criterion.

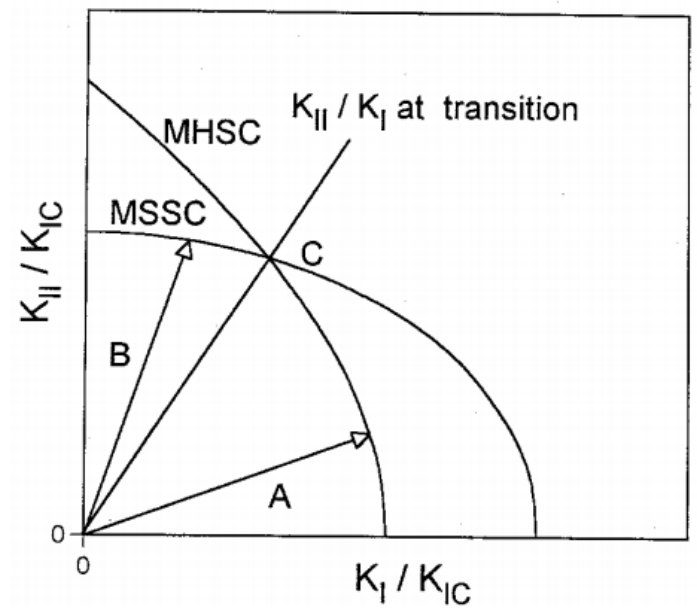

Fig. 18. 316 stainless steel MTS \& MSS vs Empirical Data [52]

$$
\frac{K_{I}}{K_{I I}}=\frac{\left.3 \cos \frac{1}{2} \theta^{*} \sin \theta^{*}+\frac{\sigma_{c}}{\tau_{c}} \cos \frac{1}{2} \theta^{* *}\left(3 \cos \theta^{* *}-1\right)\right)}{\left(2 \cos ^{3} \frac{1}{2} \theta^{*}-\frac{\sigma_{c}}{\tau_{c}} \cos \frac{1}{2} \theta^{* *} \sin \theta^{* *}\right)}
$$

The MTS and MSS criteria are useful starting points for predicting the direction of crack growth under mixed mode behavior. However, they often provide limited reliability. Experiments conducted on steels show that the crack behaves in a different manner. Instead of there being a transition point, there is a more gradual transition of the kink angle. Therefore, both the MTS and MSS modes contribute to failure. This gradual nature of crack kinking could be modeled using a strain energy approach. 


\subsubsection{STRAIN ENERGY RELEASE RATE (SERR)}

The energy release rate criterion for mixed mode fracture was developed by Nusimer. It was hypothesized that since energy release rate is considered as the "crack driving" force, then the direction of crack growth can be determined by finding the maximum possible energy release rate. The energy release rate ' $G$ ' derived in Section 2.1.2 can be represented in a form which considers a two-dimensional mixed mode crack: [53]

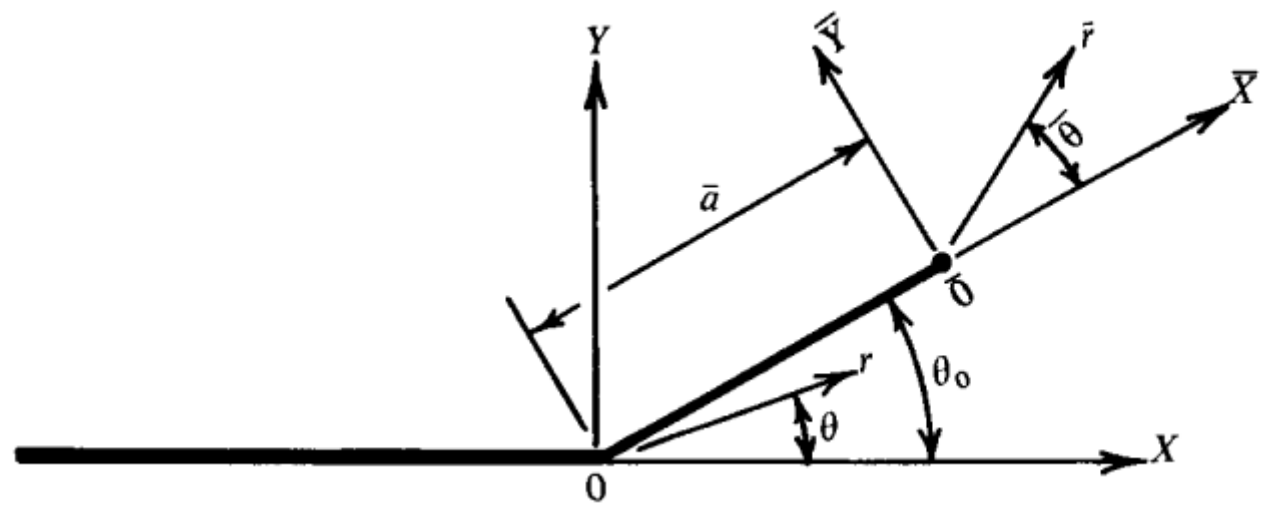

Fig. 19. Mixed mode crack branching [53]

$$
G_{o}=\frac{\pi(\kappa+1)}{8 \mu}\left(K_{I}^{2}+K_{I I}^{2}\right)
$$

The energy release rate due to the branching of the crack with direction $\theta_{o}$ is given by the following, where the stress intensity factors are for the initial unbranched crack:

$$
G_{c r}=G_{\theta o}=\frac{\pi(\kappa+1)}{8 \mu}\left(\overline{K_{I, o}^{2}}+\overline{K_{I I, o}^{2}}\right)
$$

For fracture, the energy release rate must be equal to the critical release rate which is related to the fracture toughness of the material. Furthermore, the crack will grow in a direction which the energy release rate is of maximum value. To obtain this direction, the angle $\theta$ can be obtained through the differentiation of equation (84). A more general expression for SERR is made for a three-dimensional mixed mode problem. The angle which results in the maximum energy release rate can be expressed as:

$$
\theta=\max \left[\left(K_{I}(\theta)\right)^{2}+\left(\eta_{I I} K_{I I}(\theta)\right)^{2}+\left(\eta_{I I I} K_{I I I}(\theta)\right)^{2}\right]
$$

\subsubsection{MODIFIED STRAIN ENERGY RELEASE RATE (MSERR)}

A shortcoming of the strain energy release rate criteria (1975) is that the critical energy release rate is based only on mode I loading. Since then, researchers have been exposed to a vast body of evidence indicating that the fracture toughness of metals differs when under pure mode I or mode II loading.

Kfouri and Brown built upon the energy release rate model and modified the theory to incorporate for the materials fracture toughness in different modes of loading. Assuming a mixed mode loading involving mode I and II, the modified strain energy release rate takes the form of: [54] 


$$
\left(\frac{K_{I}(\Delta \theta)}{K_{I C}}\right)^{2}+\left(\frac{K_{I I}(\Delta \theta)}{K_{I I C}}\right)^{2}=1
$$

This criterion produces an elliptical shape as plotted below.

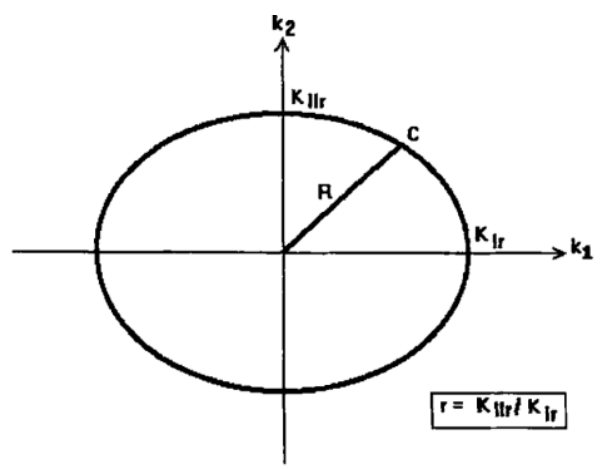

Fig. 20. MSERR Criteria [11]

To determine the critical crack growth direction, one would maximize the left-hand side of equation (86) to find $\theta$. An effective stress intensity could be found through rearrangement of equation (86). [11]

$$
K_{e q}=\operatorname{MAX}\left(\sqrt{\left(K_{I}(\Delta \theta)^{2}+\left(\frac{K_{I C}}{\mathrm{~K}_{I C}}\right)^{2}\left(K_{I I}(\Delta \theta)\right)^{2}\right.}\right)
$$

\subsubsection{CRACK GROWTH SOFTWARE}

The crack growth analysis software adopted for this work is FRANC3D. It is a fracture mechanics tool capable of analyzing crack growth for 3D structures that undergo complex loading [48], and has been previously used [8] to simulate the crack propagation for an aircraft engine fan blade attachment using the MTS theory. The software has also been used successfully for several other documented cases related to aerospace applications. The development of FRANC3D began at Cornell University in the late 1980's and the software has now been adopted worldwide in universities and aviation industries. Version 7.4 of FRANC3D is now commercialized and has much improved support and interaction with 3D FE solvers such as ANSYS, ABAQUS, and NASTRAN. The primary function of the software is to insert and extend crack tip elements in preexisting finite element (FE) meshes produced by other FE packages.
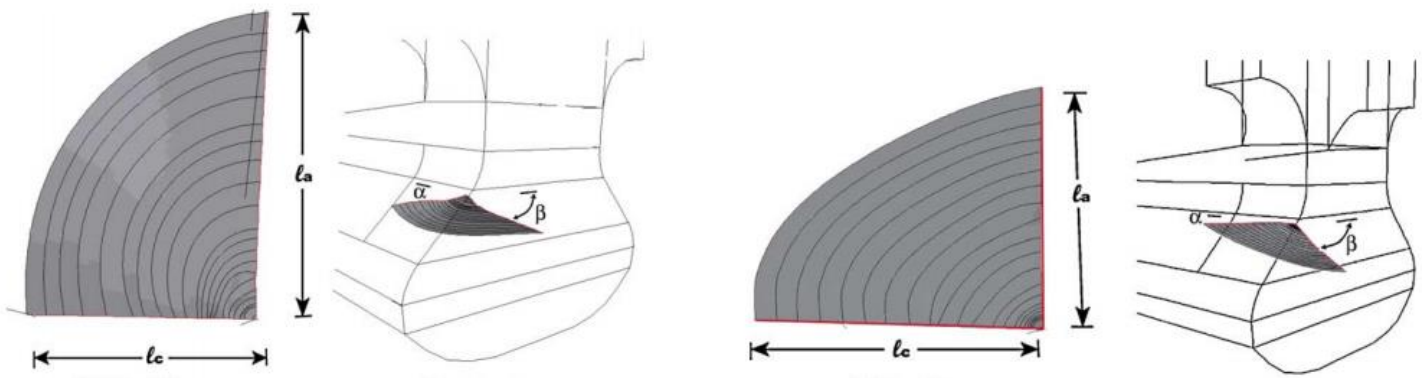

Fig. 21. - Corner flaw crack propagation using Sigma-Max method and planar extension method [8] 
Hernsehll and Barsoum [55] showed that it is possible to reproduce the high intensity stress field characteristic of LEFM by moving the side nodes of a quadratic element from the center to the quarter point. FRANC3D inserts quadratic order elements along the crack front to model the stress fields near the tip of a crack using higher order 3D 20 node solid quadratic elements. FRANC3D meshes the crack front using 'singular' elements so that the displacements obtained near the crack tip are representative of LEFM. Quadratic order elements are used to model the singular stress fields near the tip of a crack in a linear-elastic model. By moving the side nodes of a quadratic element from the centre to the quarter point, it is possible to reproduce a singular stress distribution [55]. The stress and displacements around a crack tip in a linear elastic body for Mode I loading are:

$$
\begin{gathered}
\sigma=\frac{K_{I}}{\sqrt{2 \pi r}} f(\theta) \\
u=\frac{K_{I}}{G} \sqrt{\frac{r}{2 \pi}} g(\theta)
\end{gathered}
$$

Where $\sigma$ is any of the stress components near the crack tip, $K_{I}$ is the Mode I stress intensity factor, $r$ is the distance from the crack tip, $G$ is the modulus of rigidity, $u$ is the displacement, and $f$ and $g$ are trigonometric functions of $\theta$. For a normal quadratic element with the side nodes at the centre point, the stress and displacement are found by:

$$
\begin{gathered}
\sigma=a+b r \\
u=c+d r+e r^{2}
\end{gathered}
$$

When moving the side nodes to the quarter point, a singularity problem occurs for the stress as the distance from crack tip is very close to zero. Therefore, this 'singular' element modification can accurately simulate LEFM behavior. FRANC3D meshes the crack front with the breakdown of elements shown in Fig. 22.

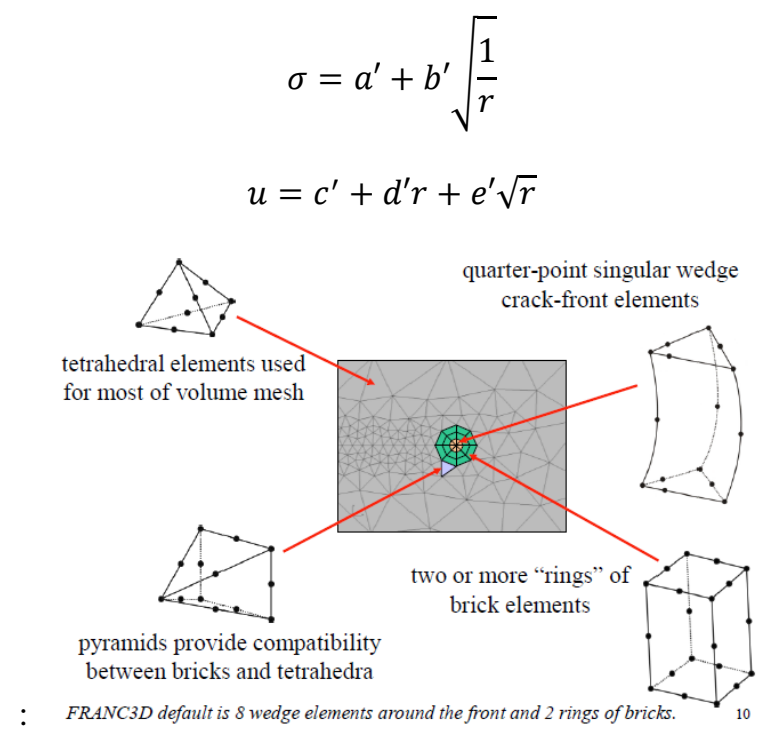

Fig. 22. Crack Front Mesh [56]

After a crack is inserted and the finite element model is solved, the stress intensity factors along the nodes of the crack front are computed from a user specified method such as the M-Integral, displacement correlation, or virtual crack closure. The M-integral method (briefly documented in Section 2.1.5.2) is built from the J-integral which is a 
path independent method of computing the global energy release rate. The computed energy release rate can then be used to calculate the stress intensity factors for the three modes of fracture. The method is well documented in [50]. Using the computed stress intensities, the local extension and kink angle for each node along the crack front are predicted for a user defined amount of crack growth iterations using one of several built-in crack kink prediction methods such as the MTS [48]. Other crack kink models include MSS or SERR explained in Section 2.1.6. The crack propagation life can then be estimated using a crack growth material curve and a stress intensity history along the resulting path of several crack advancements. FRANC3D allows for multiple crack growth curve models such as Paris equation, hyperbolic sine, and FNK model explained in Section 2.1.4. The user must enter the load history representative of the aircraft mission. The figure below shows the interaction between the FE solver and FRANC3D.

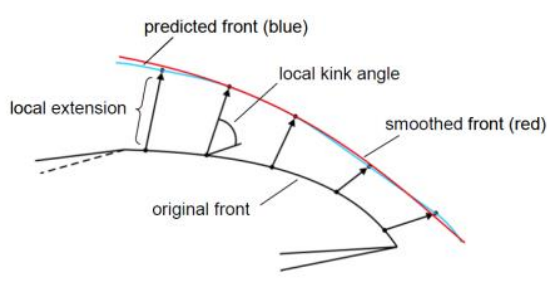

$$
\Delta a_{i}=\Delta a_{\text {median }}\left(\frac{\frac{d a}{d N_{i}}\left(\Delta K_{i}, R_{i}, . .\right)}{\frac{d a}{d N_{\text {median }}}\left(\Delta K_{\text {median }}, R_{\text {median }}, . .\right.}\right)
$$

Fig. 23. Predicted crack front [48]

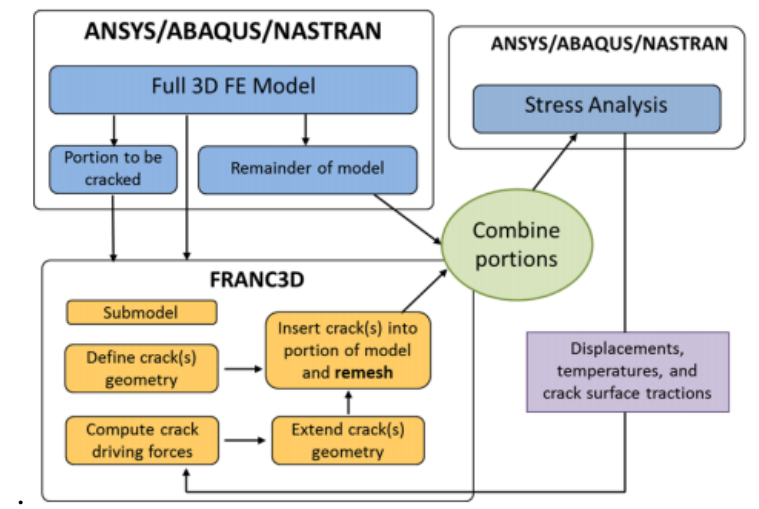

Fig. 24. FRANC3D Flow Diagram [56]

The usage of FRANC3D in the determination of stress intensity factors has been 'benchmarked' to simple classical closed form analytical methods. For a penny shaped crack/flaw embedded in a bar, the Sneddon solution from Section 0 could be used to obtain the "analytical" result. The M-Integral method in FRANC3D was also used. Results show the percent error is on the order of 0 to $0.2 \%$, depending on the refinement of the crack front [57].
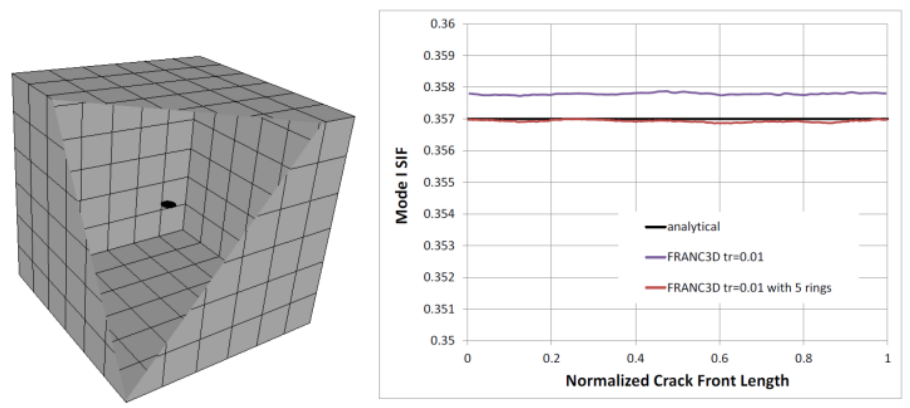

Fig. 25. Benchmark for penny crack, FRANC3D vs analytical Sneddon [57] 
FRANC3D was also benchmarked to the Raju and Newman numerical solution for a surface ellipse crack in a plate. An accuracy of 5\% was obtained for the calculation of the mode I SIF as shown below. More of these benchmarks can be found in the FRANC3D benchmark manual. [57]
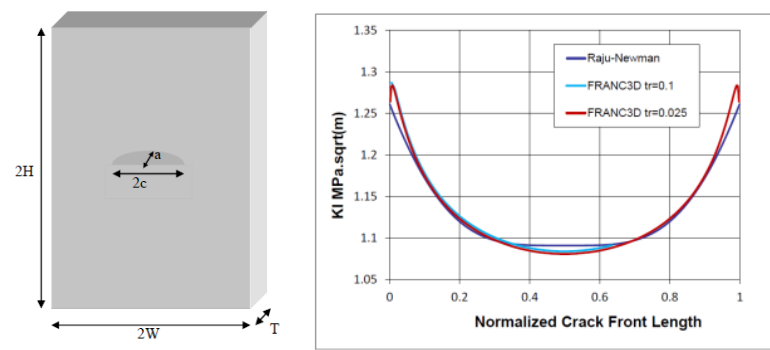

Fig. 26. Surface Crack in a plate

\subsection{FRETTING FATIGUE}

For some aircraft engine components such as a blade/disk assembly of bladed rotors, one of the controlling factors for life of aircraft engine components is fretting fatigue. Fretting occurs when two parts are in contact and subjected to cyclic loading with slip at the contact interface. Analysis of fretting is difficult due to loading at the contact interface, temperature variations, complex component geometries, anisotropic properties, residual stress, and difficult to analyze crack geometry [18]. More recent efforts have been made by Fracture Analysis Consultants Inc [18] to develop a numerical method integrated within the software to model fretting crack nucleation in addition to its existing 3D crack growth modeling capabilities [48]. The developed fretting utility leverages FE analysis software such as ANSYS to model complex contact problems. The FE solutions are passed along to FRANC3D for computation of fretting parameters using one of several built-in fretting nucleation models. The tool has been used successfully in the past to predict the total fretting cycles resulting in nucleation, as well as the location and orientation of the fretting crack for a Ti-6Al-4V dovetail fan application [18]. The aim of this literature review is to study current modeling attempts of fretting fatigue such as the dovetail specific Ruiz approach or more current critical plane approaches such as the SWT model. Using FRANC3D`s recently developed fretting nucleation prediction capabilities, these fretting models will be later tested in Chapter 4 and calibrated to spin pit test data in regard to the fretting crack nucleation site, cycles to nucleation, and remaining crack propagation life. From the studied literature, it is concluded that the modified SWT model is most appropriate for future fretting fatigue predictions as it incorporates critical plane methodology and damage effects attributed to wear.

\subsubsection{THEORY}

Fretting is the minute relative motion which may occur between clamped, static contacts when subjected to oscillatory forces [13]. Oscillating contacts, often with amplitudes as low as $25 \mu \mathrm{m}$ to $100 \mu \mathrm{m}$ [14] can significantly reduce the fatigue performance of materials relative to plain fatigue limits. This knockdown in fatigue performance accompanied with large shearing and bulk stresses promote early crack initiation and propagation within or at edge of contacts. Contrast to wear for which cracks lie parallel to the surface and are formed under plastic loading conditions, fretting cracks grow inwards normal to the contact surface, and exist in elastic stress fields [13]. Fretting fatigue is a 
difficult, costly problem associated with $\mathrm{HCF}$ and is a major life limiting factor for aero-engine blade/disk dovetail fixing assemblies [14] [19]. This literature review will focus on the fretting fatigue problem related to these mentioned dovetail slot features.

Dovetail slots commonly used for bladed fans undergo complex 3D non-proportional multiaxial stress states in which the three principal stresses change direction during loading [14]. These stress states are induced by steady and dynamic loading. Steady bulk loads $T, W$ are generated due to centrifugal forces acting on the dovetail interface at an applied angular velocity. Vibratory stresses $V$ are superimposed on steady stresses and are due to aerodynamic or mechanical excitations acting on the blade which result in oscillatory motion at the blade/disk interface [19] [3].
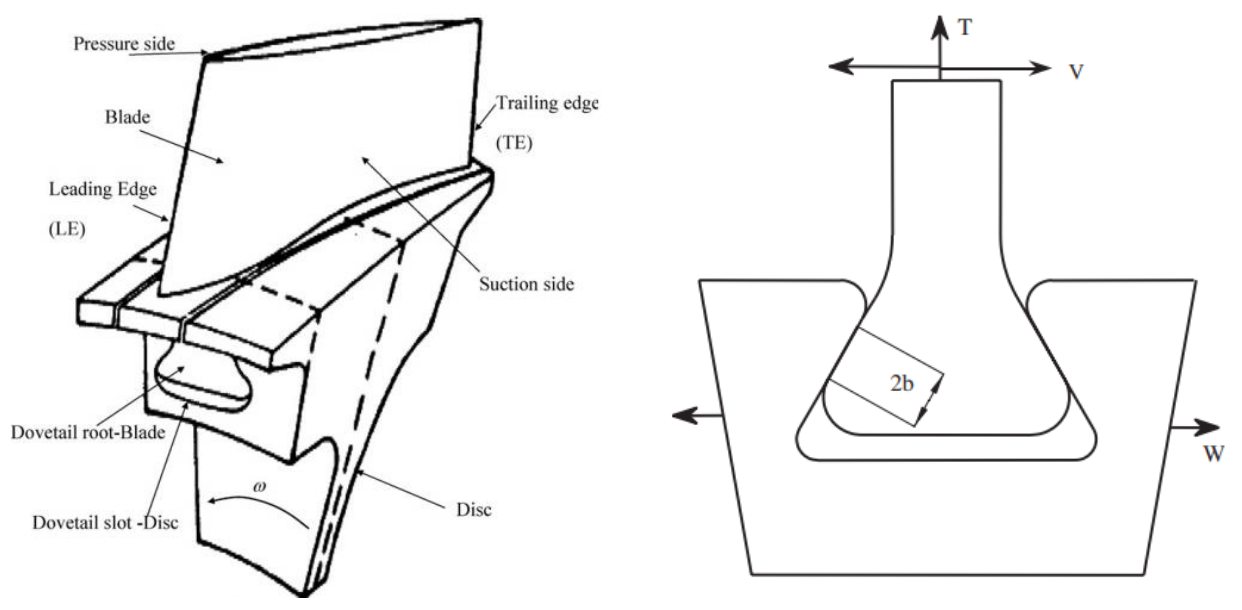

Fig. 27. (a) Typical bladed-disk rotor (dovetail joint) [3] (b) Forces acting at dovetail interface [58]

Studies show that the amplitude of relative tangential displacement $\delta$ has a very strong influence on the fretting fatigue performance and thus is an important variable in the modeling of fretting fatigue. Other variables which must be considered are those controlling the contact stress field such as COF, pressure, surface finish and loading conditions [15]. Depending on these factors, fretting damage may result in fretting fatigue, fretting wear, or both. Fretting fatigue is characterized as crack nucleation and subsequent crack growth in the occurrence of fretting and externally applied bulk loading whereas fretting wear is permanent loss of material [15] [17]. The former describes damage due to fretting when fatigue is the primary damage mechanism.

Contact conditions during fretting may be described by hysteresis loops depicted in Fig. 28 (a) which relate the applied tangential force $\mathrm{Q}$ to tangential displacement. The area inside the hysteresis loops represents the amount of frictional work dissipated. For partial slip, a portion of the body is sticking whereas the contact edges experience slip, resulting in narrow hysteresis loops which remain closed for all cycling conditions. Between the partial and gross slip regimes there is a stick-slip regime, where there is an initial gross slip followed by a transition to partial slip for subsequent cycling conditions [16]. Gross slip is characterized with very wide hysteresis loops which can also be deformed due to drop in tangential force attributed to wear effects [31]. Vingsbo et al. [31] also identifies a stick regime, where there is very limited surface damage with no fatigue crack formation observed.

Fretting maps [31] were developed as a tool to determine the fretting regime. As depicted in Fig. 28 (b), depending on the magnitude of tangential slip, fretting may result in cracking or wear. From the fretting map, two 
main regimes of slip are identified. The first is partial slip regime, where tangential displacement amplitudes range from $0 \mu \mathrm{m}$ to $50 \mu \mathrm{m}$ [15]. In the partial slip regime, as tangential force increases, the relative tangential slip increases. However, at a certain point, the ratio of tangential to normal force becomes larger than the COF, resulting in the entire contact interface to undergo gross slip for which tangential displacements range from $100 \mu \mathrm{m}$ to $250 \mu \mathrm{m}$.
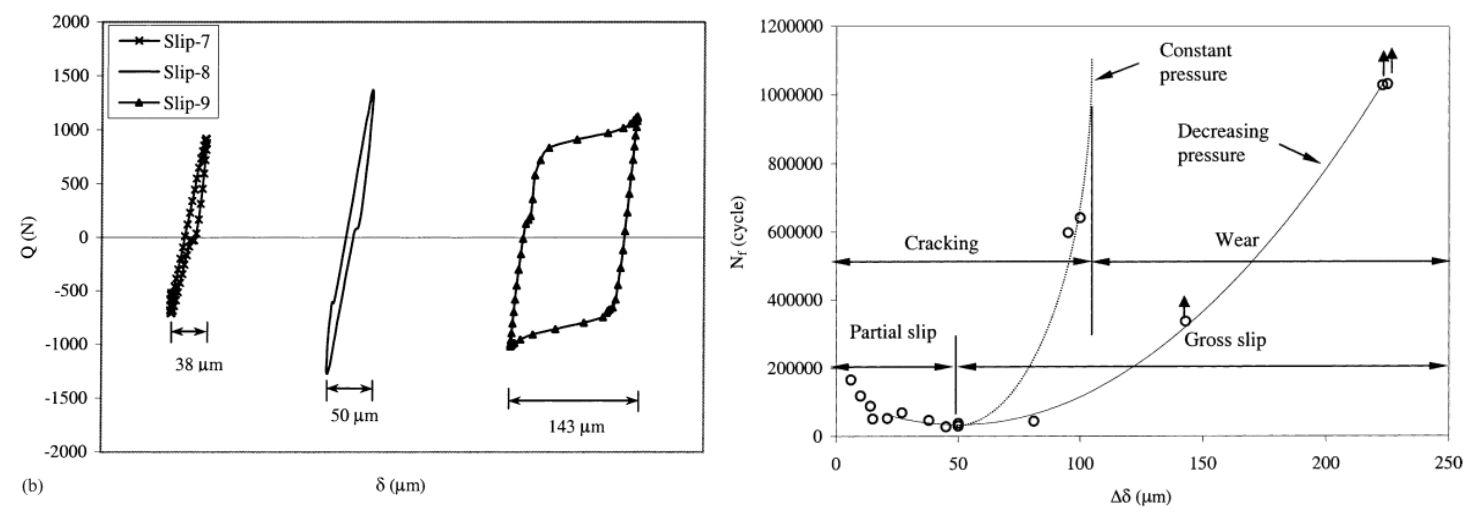

Fig. 28. (a) Partial versus gross slip (b) Fretting map [15]

The fretting map Fig. 28 (b) shows that fretting life decreases with increasing amplitude in the partial slip regime up to a certain point. After which, additional increase in tangential displacement results in an increase in fatigue life since cracks nucleated at the surface are eliminated by simultaneous wear [31]. In the gross slip regime, very high wear rates lead to plastic conditions at the contact edges which can leave material in a permanent compressive state, leading to a less likely chance of growing a nucleated fretting crack [17]. Fretting fatigue is the dominant damage mechanism when amplitude of tangential displacements are between $0 \mu \mathrm{m}$ to $100 \mu \mathrm{m}$, characterized by partial slip or mixed stick slip regimes. However, after $50 \mu \mathrm{m}$, the effects of wear become prevalent resulting in the fretting fatigue life to improve with increasing tangential displacement. An explanation for an improved fretting fatigue at higher slip amplitudes is that embryo cracks may be worn away before having enough time to grow due to a rapid increase in wear rate [17] [32] . Fretting wear takes place through abrasion, adhesion or delamination. Titanium alloys have been found to wear through delamination, in which cracks are formed parallel to the surface removing material in a platelike manner [16].

When subjected to both fretting and fatigue, crack nucleation is accelerated due to a knockdown in fatigue performance of the material. The knockdown has even been measured to be as high as 74\% [16]. Cracks initiated during fretting are more problematic compared to other types of cracks as they are not visible but instead hidden by the contact of the two bodies. The fretting fatigue life of components is controlled mostly by fretting whereas subsequent long crack propagation or arrest is controlled by bulk stresses. Initially, at the contact area, crack grows under shear mode II conditions due to very high frictional forces before turning perpendicular to the max tensile stress mode I [16].

Fretting fatigue displays many important factors which are distinct from plain fatigue. For instance, in fretting applications, there are very high localized stress gradients at the edges of contact, much more severe than other common features such as notches or bolt holes. Furthermore, the loading is non proportional at the area of contact, 
and as such, the crack depth grows away from the contact surface, it experiences a varying load ratio [32]. Localized surface damages at the asperity level also play a role in acceleration initiation of cracks. For higher slip amplitudes, there are a higher number of asperity passes [32] which can enhance the opportunity for crack nucleation.

Crack nucleation occurs at the micro level on a scale of a few grains in a non-homogenous anisotropic imperfect material where it is generally agreed upon that cracks nucleate through a shearing action along slip bands in a material. These slip bands are often observed to coincide with a plane corresponding to maximum shear strain amplitude. Thus, early fretting experiments attributed fretting cracks due to the frictional force. Further works later showed that fretting crack nucleation is also dependent on the interaction of the shear stress with the bulk tensile stresses. Shear stresses are of higher importance for early stages of crack growth, where cracks grow in an oblique manner before turning normal to the bulk tensile stress [14] [16]. Oblique cracks can grow as large as $10 \mu \mathrm{m}$ to $3000 \mu \mathrm{m}$ before turning. The location in which the crack trajectory changes corresponds to a location where contact induced stress gradients die out, thus no longer contributing to the advancement of the crack.

Damage due to fretting is difficult to model since both fatigue and wear occur concurrently with competing effects [16]. This is why most modeling attempts to fretting fatigue are focused on conditions where one of the damage mechanisms is dominant. More advanced modeling methods employ strain based fatigue and critical plane methods to predict fretting crack initiation life. Critical plane approaches can also predict the initial crack orientation. Critical

plane theories are built on an understanding that damage accumulation is a combination of a certain strain and stress on a specific plane. These theories will be later studied in more depth.

With the aid of powerful finite element modeling tools, researchers have spent time trying to combine the effects of both the wear and fatigue. In finite element approaches, mechanisms involved in the fretting phenomenon such as wear, adhesion, and asperity deformation are not considered in the assumptions made for the properties of the interface of the two components. However, these mechanisms can result in a COF which is much higher than initially predicted. Thus, using a higher value of COF has often resulted in a better correlation of analytical and experimental results [59]. Using a higher $\mathrm{COF}$ results in a drastic decrease in nucleation life by accounting for stress concentrations at the fretting interface due to asperity interactions and micro-crack tip stress fields. Further to this, since fretting is an HCF related issue which is non-linear and history dependent due to friction, elastic material properties have been traditionally used along with three complete loading cycles for stabilization of the partial slip cycles [59].

\subsubsection{INVESTIGATIONS OF FRETTING FATIGUE FAILURES IN AIRCRAFT ENGINES}

Researchers have made considerable effort to study and characterize fretting damage of aero-engines most commonly occuring at dovetail joints for blade-disk assemlies. However, fretting phenomenon is not uniquely limited to dovetail fixings. B.W. Lee et al. [20] studied the fretting behaviour of a tang type blade disk attachment shown below. In this configuration, fretting damage occurs when the retainer pin and tang experience oscillatory motion due to aerodynamic or mechanical exciation combined with bulk axial loading due to centripetal forces. 

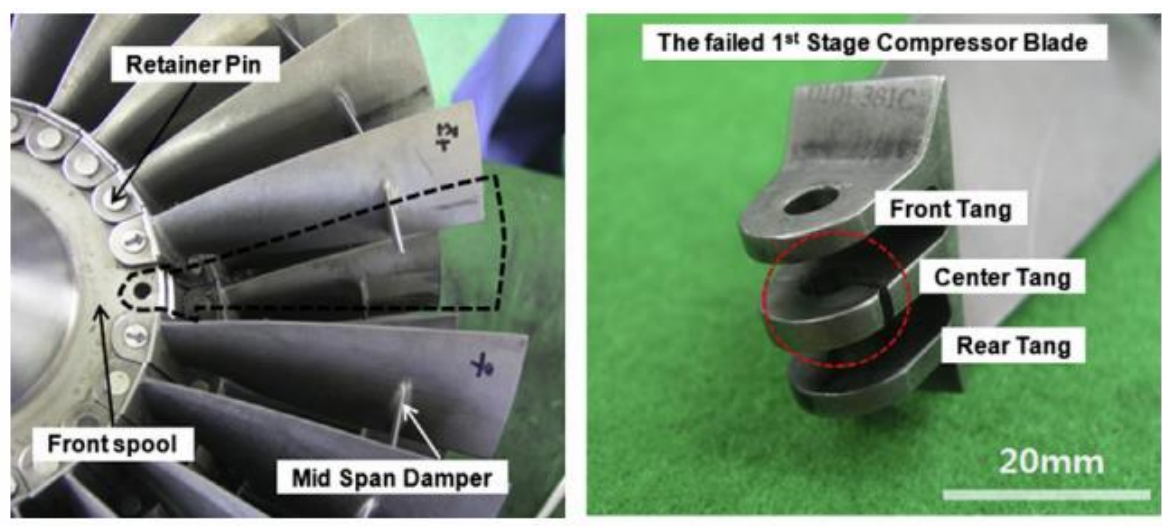

Fig. 29. 1st stage compressor assembly with failed pinhole lug [20]

Fractographic investigations were carried out to study the fracture surface of the failed tangs using Scanning Electron Microscope (SEM) combined with X-ray Energy Dispersive Spectrometer (EDS). The investigation revealed that the crack propagated due to fatigue loading until reaching a crack depth corresponding to one third of the total crack length. It was concluded, based on the noticeable debris loss at the initiation site that the crack initiated due to fretting fatigue. Fretting occurred due to aerodynamic vibration whereas subsequent crack growth was attributed to LCF loading [20].
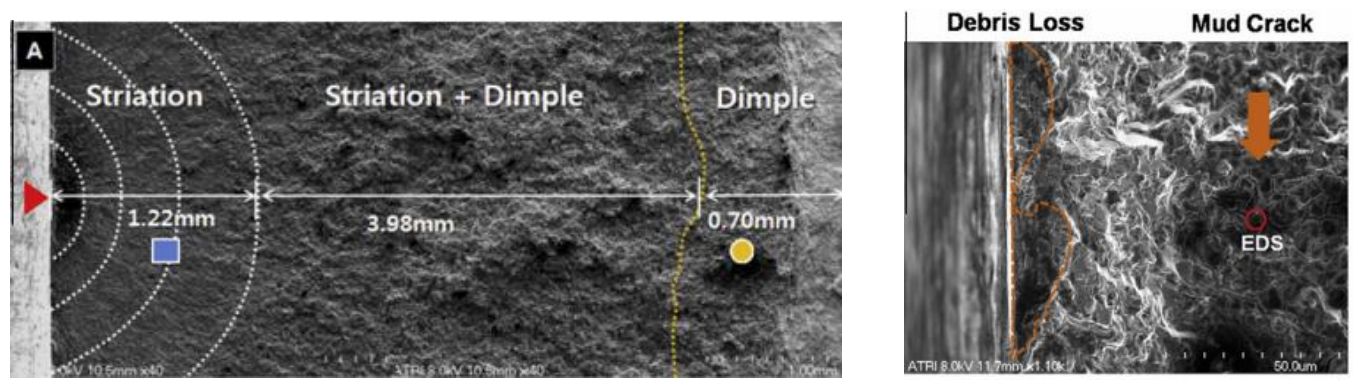

Fig. 30. Fractography of fracture surface and close up of crack initiation area [20]

H. Tang et al. [21] studied the fretting failure of two blades of the first stage low pressure turbine held together using a fir-tree dovetail slot. From visual examination, there was an obvious turning line between initiation area and propagation area shown in Fig. 31 (a). This is in agreement with previously mentioned fretting fatigue characteristics.

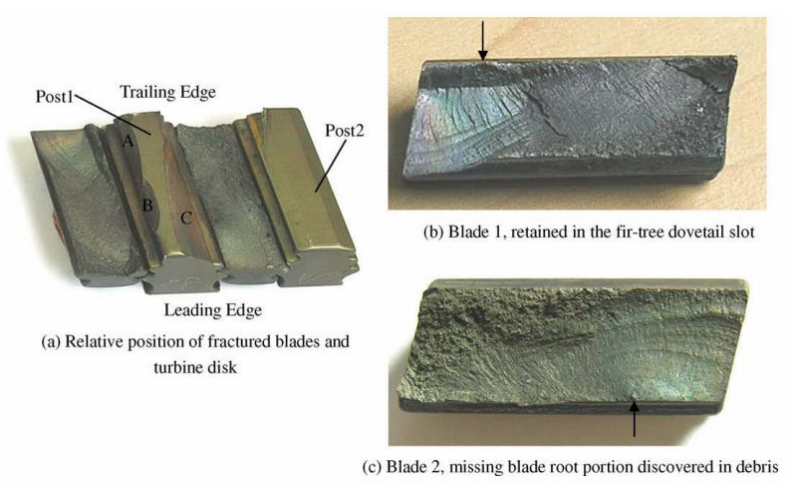

Fig. 31. Visual fracture appearance of the two low pressure turbine blades [21] 
The fracture surface of blade 1 - which was the first blade to fracture - was analyzed using SEM. It was concluded that the fracture initiated from a $\mathrm{V}$-notch and propagated as a fatigue crack as suggested by the obvious beach marks found in the interior fracture surface of the blade. However, it propagated more rapidly along the two sides with very little depth into the blade root. It was concluded based on these findings that HCF was the root cause. Wear characteristics in the form of small pits around the V-notch were present and are shown below [21].

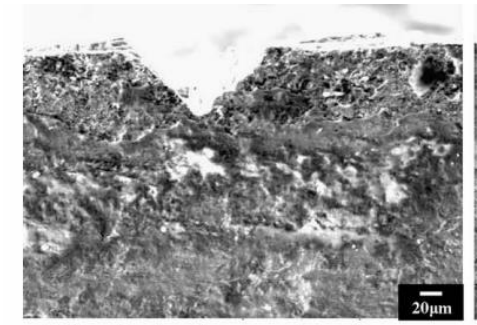

(a) Small pits close to origin site

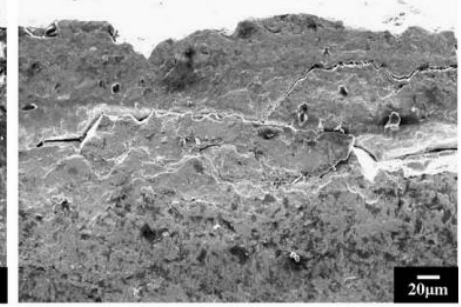

(b) Cracks far away origin site

Fig. 32. Appearance of blade 1 fracture surface [21]

S. Barella et al. similarly investigates a third stage turbine blade failure [22] comprising of a fir tree dovetail configuration of a nickel and cobalt based alloy. Although the studied metal is intended to withstand combinations of high and stress and temperature while remaining resistive to corrosion, it is not exempt from the fretting fatigue phenomenon. In operation, the turbine is exposed to two forces acting on the fir tree joint. The first is radial strain due to centrifugal loading. As angular velocity continues to increase, the disk slot opens, allowing the blade to slip by a minute amount. The second load is associated to high cycle fatigue and comes in the form of blade oscillation due to aerodynamic unsteady loads. These two loads when combined create the fretting phenomenon [22]. Eliminating or reducing the slip between the blade and the disk helps prevent fretting fatigue during the design process. Shot peening has also been effective in increasing the fretting fatigue life of components by inducing compressive stresses at the surface.

The fractographic analysis on the failed turbine blade of a thermal power plant revealed that the fracture presented brittle characteristics as there were no plastic deformations at the fracture zone. The fir tree root was split into three pieces to allow for the fracture surface to be analyzed using SEM and EDS. Three distinct stages were identified consisting of crack initiation, crack growth, and the final fracture. Brownish marks were detected at the fatigue origin site, which were suggested to be characteristic of fretting fatigue damage. The repetitive contact between the blade and steel rotor disk resulted in the accumulation of wear debris. Fretting cracks were observed under close examination of the fir tree root, revealing cracks $500 \mu \mathrm{m}$ to $2000 \mu \mathrm{m}$ across the root contact area shown in Fig. 33 (b).
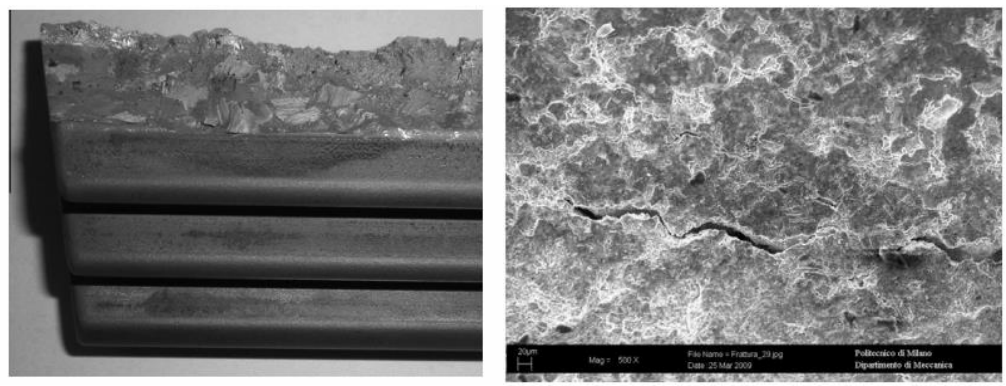

Fig. 33. (a) Brownish marks on fir tree root (b) High magnification revealing fretting cracks [22] 
D. Garcia et al. [60] attempted to analyze and characterize the fracture surface associated with Ti-6Al-4V to provide an understanding of the general physical nature of fretting fatigue cracks. Laboratory controlled fretting fatigue experiments were carried out using titanium alloy specimens until failure. The fracture surface was then analyzed using SEM with the intention of locating, characterizing, and recording fretting cracks. Fractography revealed that the fretting cracks were physically much smaller and shallower in depth compared to the contact region. Accompanied with an externally applied load, these seemingly small cracks quickly propagated to a fatigue crack. It was noticed that fretting cracks are more localized at contact area and propagate into the depth of the material through elliptical fronts depending on factors including but not limited to the stress gradient, geometry, and material properties [60]. The fracture surface of the fretting cracks were described to be smooth and featureless. From SEM, it was shown that the transition of fretting induced cracks to fatigue cracks are quite noticeable as shown below. The fretting crack region and fatigue propagation region are divided through the black dashed line [60].

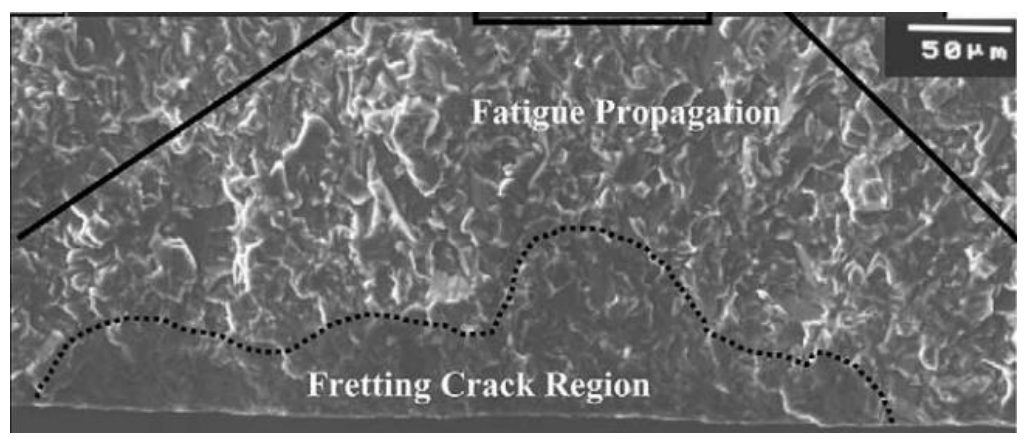

Fig. 34. Fracture surface of fretting and fatigue cracks

D. Garcia et al. [60] also states that the notion in which fretting cracks nucleate approximately 45 degrees to the contact surface for $(\alpha+\beta)$ Ti-6Al-4V is not conclusive or scientifically factual statement. Instead, it was observed that only some cracks nucleate 45 degrees to the contact, and that other cracks do not necessarily nucleate at this same angle. Thus, it was suggested that multiple fretting cracks propagate at various angles depending on the crystallographic structure of the $\alpha$ phase of the titanium alloy [60].

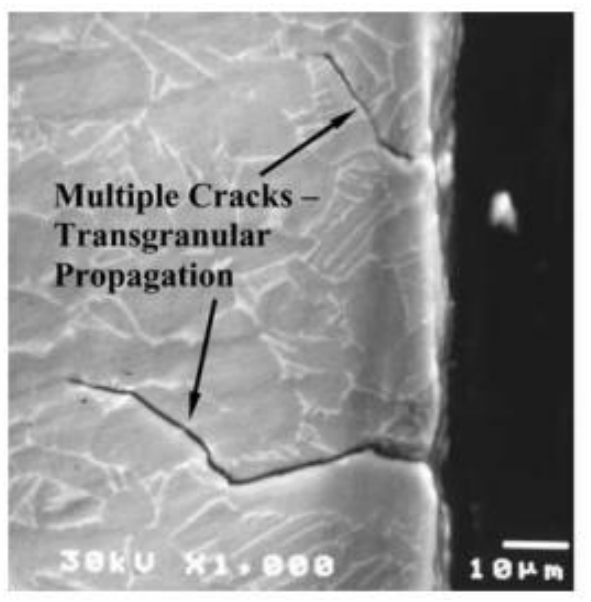

Fig. 35. Fretting crack profile using SEM [60] 
The physical nature of the fretting cracks was described to be elliptical in shape with aspect ratios less than one. This is due to steep stress gradients induced by the fretting contact resulting in accelerated crack growth along the contact surface compared to the slower crack growth along the crack depth. These stress gradients can be so sharp, often resulting in crack arrest. Lastly, it was noticed that multiple fretting cracks initiate along the contact surface. However, for the majority of the failed specimens, the fracture of the component was determined from a single dominant crack [60].

A. Hutson et al. [61] studied fretting fatigue damage using SEM to understand effects of the roughness to the damaged fretted surface. The roughness, which is associated to the asperity height and spacing reflected visual changes in the final fracture surfaces as shown in Fig. 36, however, there was no measured decrease in the residual fatigue strength.
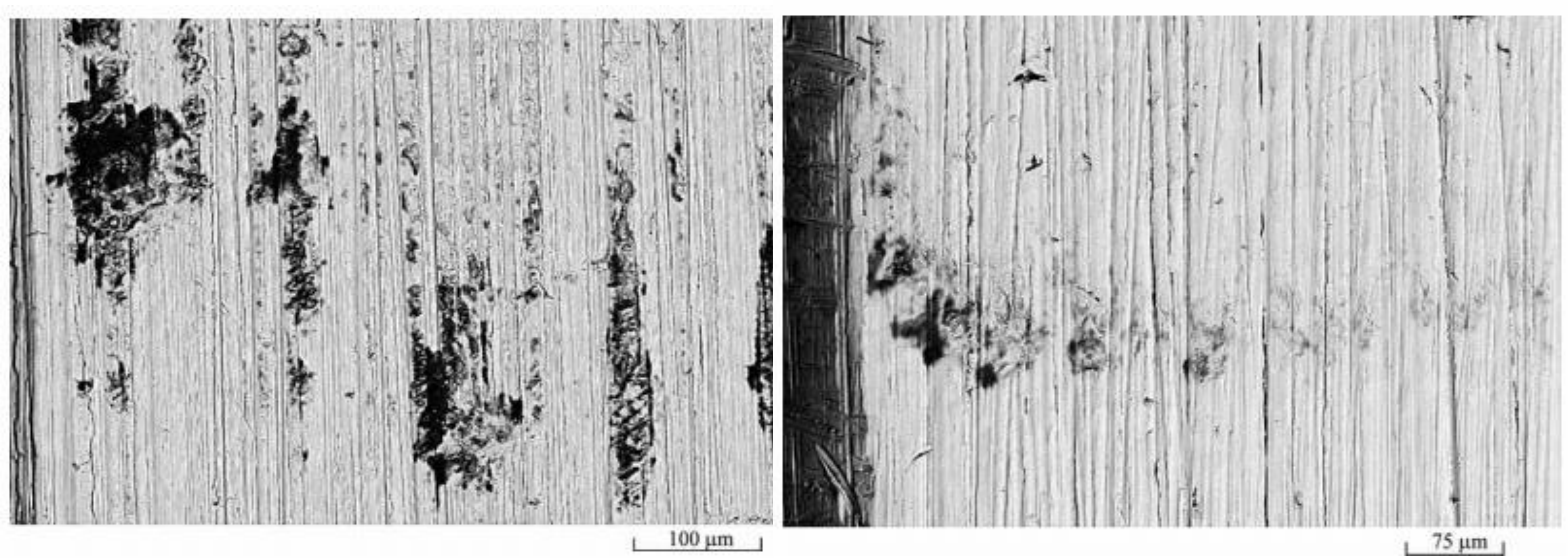

Fig. 36. Fretting damage (a) average roughness $=500 \mathrm{~nm}$, (b) average roughness $=275 \mathrm{~nm}[61]$

\subsubsection{FRETTING FATIGUE PARAMETERS}

The overall fatigue process starts at the micro level, where it is believed that cracks initiate on persistent slip bands which further coalesce into an initial micro crack. Thus, critical plane parameters have been widely accepted as a means of predicting fretting fatigue initiation life, location of crack, and orientation of the crack [14]. To implement any of these critical plane approaches on a fretting fatigue problem, a complete picture of the cyclic stress and strain field under appropriate contact definitions is required [14]. This can be obtained using finite element tools such as ANSYS.

Dovetail fan slots under centrifugal and oscillating aerodynamic loading undergo complex non proportional multiaxial stress states. From these complex stress states, critical plane-based methods may be used to characterize fretting fatigue when fatigue damage dominates the effects of wear [14]. Critical plane fatigue methods find a plane for which a maximum value for a certain combination of stress and strain occurs. Stress transformation equations may be used to compute the stresses for all planes using equation (107). Critical plane methods are largely based on modifications of the Coffin-Manson equations and consider effects of mean stress. The SWT model [23] represented by equation (108) is a critical plane parameter based on a crack nucleating on a plane where the combination of the normal strain range and maximum normal stress is maximum. For the SWT parameter, $\Delta \varepsilon$ is the normal strain range for a given plane and $\sigma_{\max }$ is the maximum normal stress on the same plane. Critical plane approaches such as the 
SWT have been successfully used to predict fretting fatigue initiation life using plain fatigue data for Ti-6Al-4V titanium alloy once the applied loading is corrected for effects of contact [24].

$$
\begin{gathered}
\sigma=\frac{\sigma_{x x}+\sigma_{y y}}{2}+\frac{\sigma_{x x}-\sigma_{y y}}{2} \cos 2 \theta+\tau_{x y} \sin 2 \theta \\
\frac{\Delta \varepsilon}{2} \sigma_{\max }=f\left(N_{f}\right)
\end{gathered}
$$

A. Fatemi et al. [29] extended on the critical plane approach by considering cases where crack formation on shear slip band planes consumes most life. This approach is referred to as the FS parameter, and is represented by equation (97) where $\Delta \gamma$ is the shear strain range, $\sigma_{n}^{\max }$ is the maximum normal stress on the same plane, $\sigma_{y}$ is the yield stress, and $k$ is a material constant. It is noted however by Fatemi that the nucleation mode must be identified so that an appropriate fatigue parameter can be applied. In fretting fatigue, since the localized tensile stresses dominate the characteristic initiation site, it is believed that the SWT is a more logical macroscopic model for predicating nucleation life and location.

$$
\left(\frac{\Delta \gamma}{2}\left[1+k \frac{\sigma_{n}^{\max }}{\sigma_{y}}\right]\right)=f\left(N_{f}\right)
$$

C. Lykins et al. [24] also uses the critical plane approach to predict fretting fatigue by creating a fatigue parameter which considers the plane of maximum shear stress range. The proposed parameter was effective in characterizing fretting crack initiation for Ti-6Al-4V and is represented by equation (98) where $\tau_{\text {max }}$ is the maximum shear stress on the critical plane, $R_{\tau}$ is the shear stress ratio on the critical plane, and $m$ is a fitting parameter. The maximum shear stress method was inspired by the notion that for ductile materials, cracks form due to the creation of slip bands due to shear motion of crystal planes. The comparison of the analytical and experimental results showed that the fretting crack initiation was governed by the maximum shear stress and that the location of maximum shear stress amplitude coincided with the observed crack location. Lykins noted however that the plain fatigue data must exist under similar non-proportional multiaxial loading conditions which occurs under fretting fatigue. The maximum shear stress model was effective in predicting the number of cycles to crack initiation, crack location, and orientation along the contact surfaces [24]. An interesting observation made in this study was that the COF increased from 0.3 to 0.5 over 10,000 fretting cycles before approaching a constant value. This supports the need proposed [59] to prescribe a higher COF when modeling fretting fatigue.

$$
\tau_{\max }\left(1-R_{\tau}\right)^{m}=f\left(N_{f}\right)
$$

The final experimental results agreed with aforementioned behavior of fretting fatigue in which cracks originally grow in a mode II manner governed by high shear forces at the contact face before turning perpendicular to the applied axial stress in a mode I manner. In this work, it was observed that the crack turned from mode I to mode II after reaching a size of $100 \mu \mathrm{m}$ [24]. 


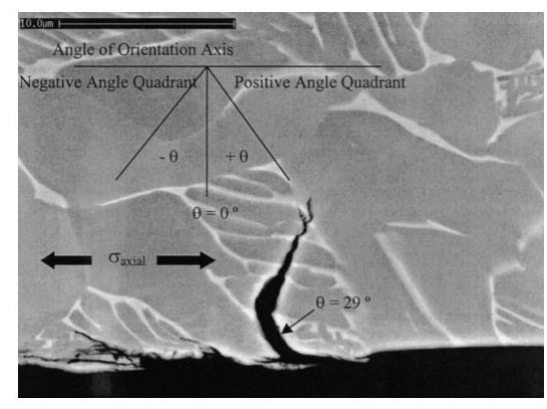

Fig. 37. Crack growth path in tested specimen [24]

C. Ruiz et al. [30] tackled the fretting problem with a different approach by studying the dovetail joint between a blade and disk using experimental and finite element results. A fretting parameter was developed which considered effects of magnitude of slip $\delta$ as it was observed that cracks nucleate at locations often associated with larger slip amplitudes. The method which was developed based on experimental results considered the bulk tensile stress $\sigma_{T}$, shear stress $\tau$, and tangential slip amplitude $\delta$ as represented by equation (99). The shear stress and slip amplitude when multiplied together represent an arbitrary frictional work term. This method is unique to the critical plane models as it considers both the fretting wear and fatigue damage effects. The Ruiz model was found to correlate well to the studied dovetail fan joint. The Ruiz parameter is unique to the mentioned critical plane parameters as it does not depend on peak stress alone. Rather, the fretting damage is embedded into the parameter.

$$
k=\sigma_{T} * \tau * \delta
$$

Ruiz also studied the effect of fretting at various applied load conditions and for varying COF. Firstly, it was observed for a constant COF that when the pulling loading of the blade increases, the dovetail opens up more, allowing for an increase in slip. Secondly, as COF decreased, the maximum normal stress increased as depicted in Fig. 38 to maintain equilibrium while the tangential stress decreased [30]. Further to this, the position of the maximum tangential stress was always below the edges of contact. It was also observed that the crack always started 37 degrees from the root, not necessarily corresponding to where the tangential stress reaches its peak. Finally, Ruiz saw that for a given $\mathrm{COF}$, the value of the maximum tangential stress increased as the applied pulling load of the blade was increased, but not as significantly compared to that when varying COF [30].

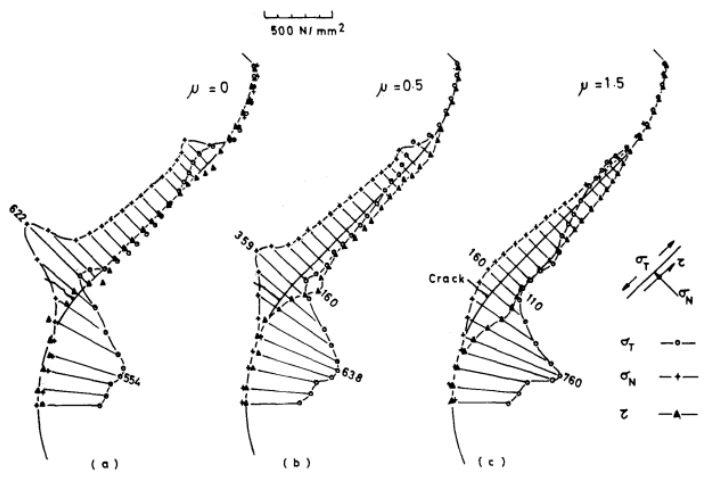

Fig. 38. Interface stresses for $20 \mathrm{kN}$ loading [30] 
Ding et al [33] sought for a unified model to account for fretting damage by considering its' two damage mechanisms, namely fretting wear and fretting fatigue. Although critical plane approaches have been successful in modeling fretting fatigue, they lack a physical parameter relating damage attributed to fretting wear. Conversely, the Ruiz parameter accounts for damage due to wear but lacks a damage parameter based on critical plane methodology. Thus, a new modified SWT parameter was developed to incorpoate aspects of both critical plane methodology and damage due to wear. The parameter is studied in depth in Chapter 4.

The modified SWT parameter assumes that fretting fatigue relies on the conjoint action of frictional work $\tau \delta$ which results in nucleation of small crack below the contact surface, and the SWT parameter which drives the growth of the crack. The modified SWT model considers both the SWT parameter and a new damage parameter due to wear $D_{\text {fret }}$. The frictional work $\tau \delta$ imbedded in the wear parameter accelerates crack initiation. Thus, the predicted fretting fatigue life can be reduced even under a small SWT parameter if the wear parameter is large. The proposed model also incorporates a threshold limit $(\tau \delta)_{t h}$ which distinguishes fretting damage to either fretting fatigue or fretting wear effects. As the frictional work approaches the threshold value for large slip conditions, fretting wear becomes more probable than fretting fatigue. Thus, when $\tau \delta>(\tau \delta)_{t h}$, embryo cracks are worn away before having enough chance to grow. The $m, n$, and $(\tau \delta)_{t h}$ are material contants which are determined by experiments. The fretting damage parameter and the predicted fretting fatigue life calculated using a modified SWT approach is graphically represented Fig. 39 (a) and (b) [33] for an aero-engine spline coupling application.
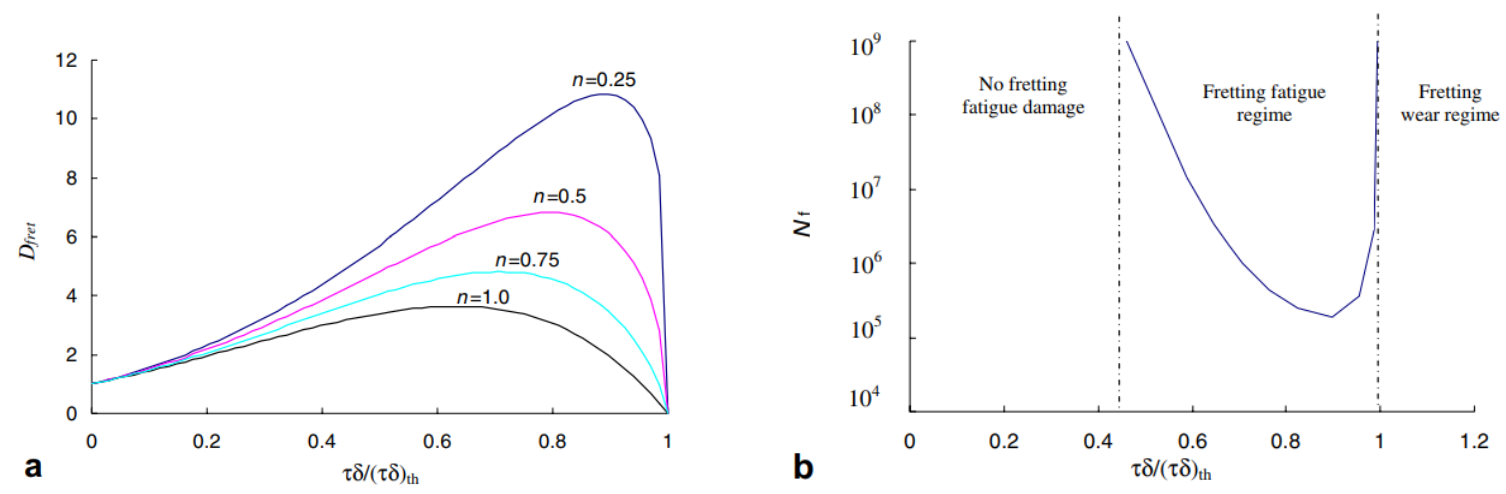

Fig. 39. (a) Damage due to fretting (b) Predicted fretting life according to modified SWT approach [33]

It is clear that as frictional work increases, $D_{\text {fret }}$ increases signifcantlly. For instance, using $n=0.25$, the fretting damage reaches a maximum value when the frictional work is $90 \%$ of the threshold. After which $D_{\text {fret }}$ drops to zero as the effects of wear begin to dominate fretting fatigue. Furthermore, the fretting fatigue life shows a rapid as frictional work increases. However, when approaching the frictional threshold, effects of wear take become pronounced resulting in a rapid increase in fatigue life due to embryo cracks being worn away before growing to failure. This method effectively captures the effects of slip on the fretting fatigue life, and closely resembles the fretting maps introduced by Vingsbo et al. [31]. It was concluded that the modified SWT led to more accurate predictions of the fretting fatigue life and location of fretting induced cracks for the aero-engine spline coupling analyzed but needs more extensive validation [33]. 


\subsubsection{MODELING FRETTING FATIGUE}

Several works have applied multiaxial critical plane approaches to predict fretting fatigue initiation life with great success. Work done by Araujo et al. [25] utilized the SWT and FS parameters by averaging the fatigue parameters over a critical volume rather than looking at distinct stressed points on a surface. By doing so, satisfactory results were achieved which correlated well to lab controlled tests for Ti-6Al-4V alloy. The averaging approach is depicted in Fig. 40 for a fretting pad test setup. First, the cyclic stress field is calculated over a mesh. The stress and strain components over each volume of element are then averaged using the distinct stressed points at each load step. The SWT and FS parameters are then calculated using the volume averaged stress and strains for each element. The global maximum SWT and FS parameters correspond to the location of crack initiation. Since the parameters are based on critical plane methodology, the critical plane orientation can also be obtained. When an averaging approach is not used, the use of critical plane parameters result in overly conservative predictions. Averaging the fatigue parameters over a critical volume is done as a basis to reflect the size effect, where it was noticed that the fretting fatigue life varies considerably with contact size. By using the average approach, the initiation parameter relies on enough volume of highly stressed material to propagate a short crack into adjacent grains [25].

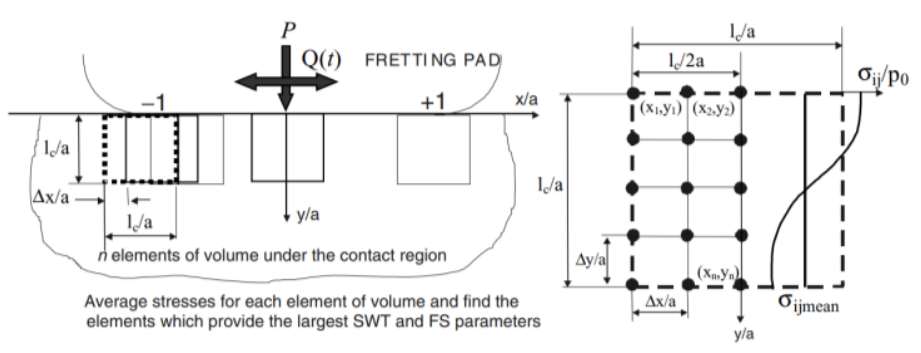

Fig. 40. Averaging method [25]

Lykins et al. [26] studied the application of various fatigue parameters for predicting fretting fatigue crack initiation. The strain life parameter, the maximum strain corrected for mean stress, maximum principal strain corrected for principal strain ratio, the critical plane SWT parameter, the FS parameter, and the Ruiz parameter were utilized in this study. The intention was to evaluate the effectiveness of these parameters in predicting the number of cycles to initiate a crack, and the corresponding crack initiation location. Fig. $\mathbf{4 1}$ shows the experiment setup used in this study. The normal load $P$ was kept constant while varying the axial load. The fretting fatigue tests were conducted under constant amplitude at a loading frequency of $200 \mathrm{~Hz}$ over a wide range of axial stress and axial stress ratios. The tangential load $Q$ is a function of the axial load and the springs used to mount the fretting pads. Plain fatigue data was also obtained to compare the fretting fatigue and plain fatigue life.

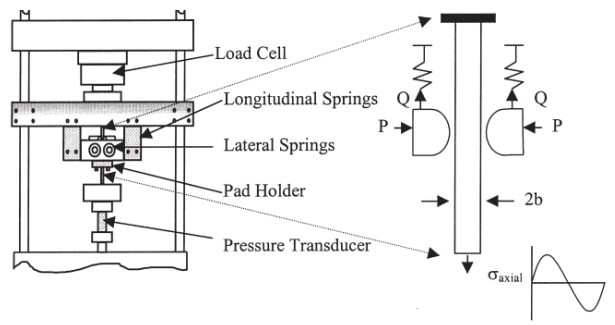

Fig. 41. Fretting fatigue experiment setup [26] 
The fretting fatigue tests were conducted until rupture of the specimens. The fatigue striations were then counted on the failure specimens to determine the propagation life. The initiation life was then be found by subtracting the propagation life from the total cycles to failure. The final averaged crack propagation life starting from a $760 \times 380$ $\mu \mathrm{m}$ crack was found to be $11 \%$ of the total life of the specimen. Thus, nearly $90 \%$ of the fretting fatigue life of the specimen was associated to the crack nucleation phase [26].

An elastic finite element model was constructed shown in Fig. 42 using ABAQUS to determine the stress, strain, and displacement fields at the location of contact. The loads were applied in the model in a total of three steps. First, the normal load $P$ was applied to establish contact. Secondly, the maximum tangential load and maximum axial stress were applied. Finally, the minimum tangential load and axial stress were applied. Several cycles of these maximum and minimum load conditions were performed to investigate the non-linear hysteresis effects due to frictional slip behavior. It was found that the use of multiple cycles did not alter the results after the first cycle. The element size required for convergence at the contact region was determined to be $6.2 \times 6.2 \mu \mathrm{m}$ [26]. The finite element results indicated the highest stress concentration at the trailing edge of contact, which also corresponded to the crack initiation location from experiments.

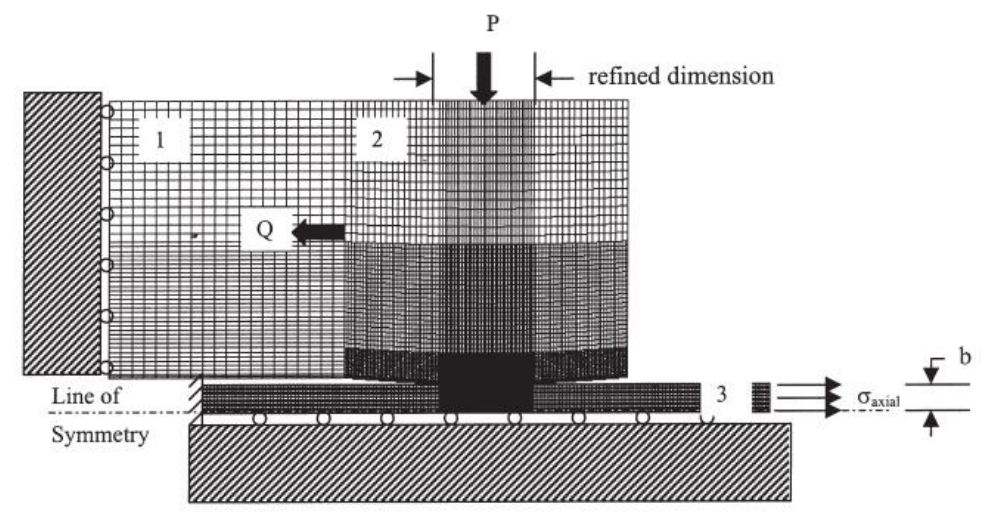

Fig. 42. Finite element model [26]

Using the stress, strains, and displacements at the contact interface, various parameters were employed to predict fretting fatigue. From all studied fatigue parameters, the strain based life parameter was not capable of predicting cycles to crack initiation under various stress or strain ratios whereas all other parameters including the SWT were equally effective in doing so. Furthermore, all of the studied parameters predicted failure at the trailing edge of contact, which agreed with experimental results. The FS parameter showed some difference between plain fatigue and fretting fatigue data in comparison to the other parameters. However, the FS parameter was effective in predicting location of crack initiation. The Ruiz-Chen parameter also predicted location of crack initiation at the trailing edge which agreed with experimental results [26]. Several other conclusions were drawn from this study. Firstly, it was determined that the maximum strain amplitude at the contact interface was an important parameter in predicting crack initiation location. Further to this, when the applied loading was corrected for the contact stress field, the Ti-6Al-4V behaved in a similar manner in both fretting fatigue and plain fatigue conditions [26].

W. Siang Sum et al. [27] developed a finite element based critical plane approach which incorporated the SWT multiaxial fatigue critertion. In this study, the cylinder-on-flat fretting contact was used to predict failure location, 
orientation, and the fretting fatigue life. The developed method was then applied to more complex spline coupling features which are suscpetible to fretting fatigue. Volume averaging approach developed by Araujo et al. [25] was required to match the numerical with experimental results. The overall process for determining the critical plane of the specimen is shown in Fig. 43. First, the stress and strain data are obtained from the finite element analysis for each loading cycle. The average stress and strains then computed for each element. Two-dimensional stress and strain transformations are then done to find the stress and strains at various critical plane angles, from which the SWT damage parameter is calculated. The global maximum SWT parameter and the angle for which it occurs corresponds to the location of crack initiation and orientation angle [27].

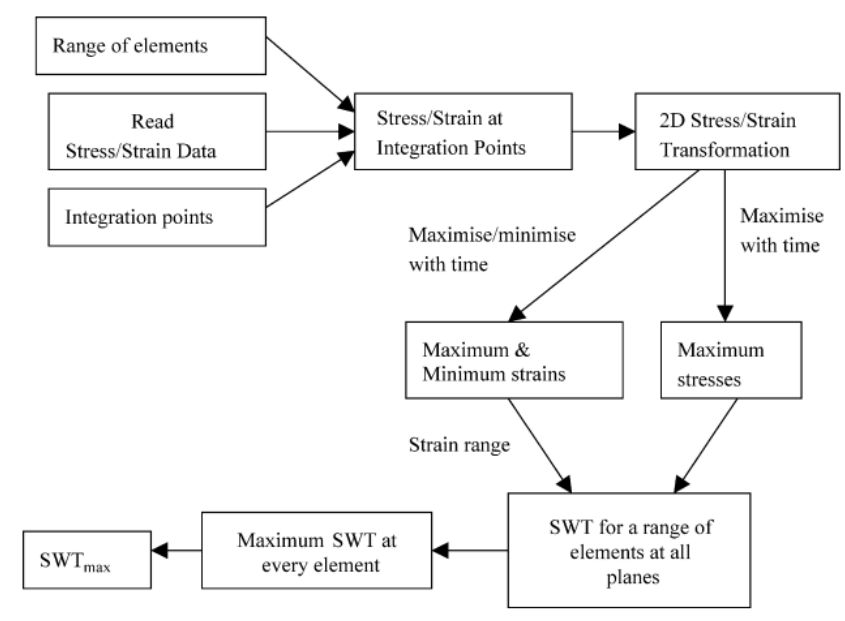

Fig. 43. Procedure used to find maximum SWT value [27]

This approach was adopted for a cylinder-on-flat fretting test as shown in Fig. $\mathbf{4 4}$ in which three loadings are applied which consist of a normal clamp load $P$, a cyclic shear load $Q$, and a cyclic bulk stress $\Delta \sigma$. Four load steps were used for the analysis. The first load step was used to apply the normal clamp load. Second load step applies a positive shear load with a positive bulk stress. The third load steps removes the shear load and bulk stress, only leaving the normal load. The final load step applies the shear load and bulk stress in the opposite direction to that in the second load step. The finite element predictions of the contact solution showed excellent agreement with the well-known Hertzian solution. The shear traction obtained from the model is shown for each load step in Fig. 45 (a). The subsurface stress distributions across the contact region at the end of load step 2 is shown in Fig. 45 (b).
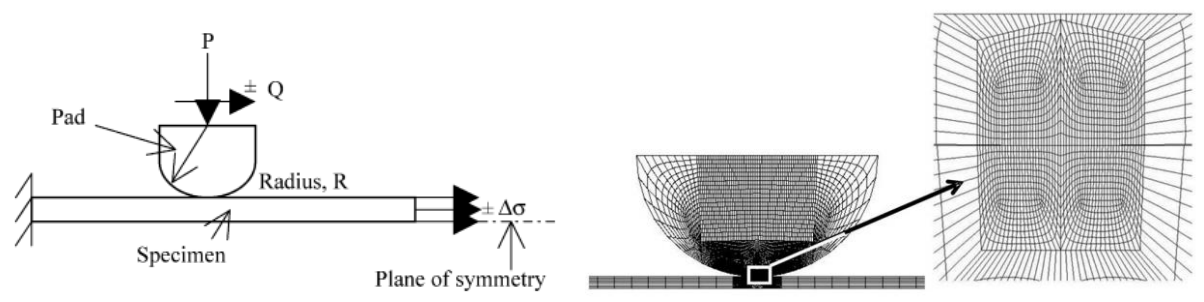

Fig. 44. Cylinder-on-flat fretting test and finite model [27] 

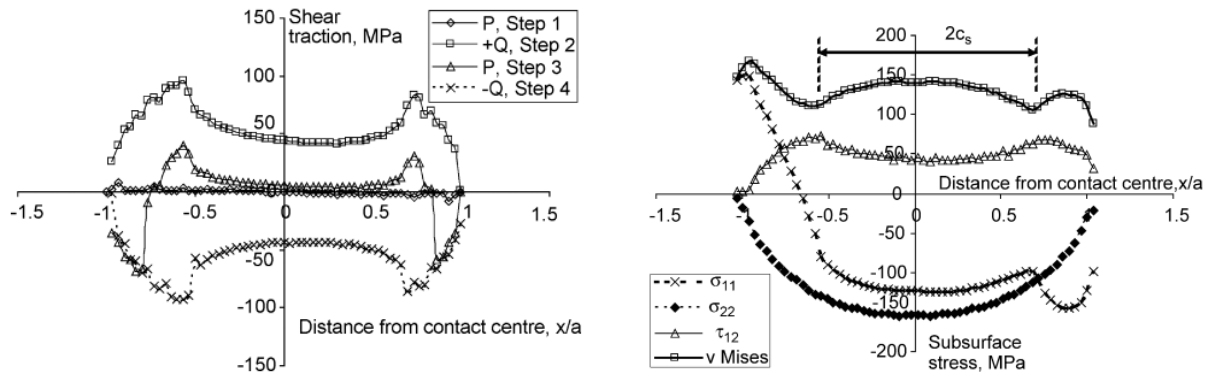

Fig. 45. (a) Shear traction distribution at different load steps (b) subsurface stress distributions at step 2 [27]

The distribution of $\sigma_{22}$ and $\tau_{12}$ correspond to the contact pressure and shear traction distributions. The tensile stress $\sigma_{11}$ peaks at the trailing edge of contact. It is believed that cracks are expected to initiate at the trailing edge since this is the location where tensile stresses are largest. However, the shear tractions are highest at the stick slip interface. Thus, it is expected that the crack either occurs at the edge of contact, or at the stick slip interface [27]. Based on the procedure of finding the maximum SWT value depicted in Fig. 43, the distribution of the SWT critical plane parameter was obtained along the contact region and shown in Fig. 46. It was found that the maximum SWT value occurs at the trailing edge of contact at angle of 10 degrees measured from the vertical instead of the slip zone. This is since at the slip zone, the SWT parameters are negligible due to large biaxial compressive stress states and due to the shear stresses being relatively small in magnitude [27]. The predicted crack location, orientation, and fretting fatigue life correlated very well with test data.

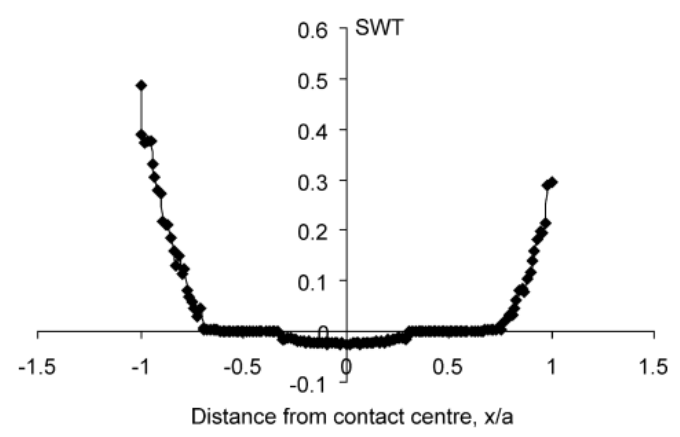

Fig. 46. Distribution of maximum SWT parameter [27]

W. Siang Sum et al. [27] used this developed numerical procedure to predict fretting behavior of aero-engine complex spline couplings. The procedure was intended to improve older lifing methodologies that were based on maximum principal stress criteria and LCF curves. The method was able to correctly prediction the location and approximate orientation of several of the cracked cases. For this work, a constant COF was used with a value of 0.3. However, several works have shown that this value might vary with increasing number of cycles. It was concluded that a more advanced lifing methodology would therefore also consider the evolution of the COF [27].

Up to this point, the modeling of fretting fatigue has been mostly studied for cylinder on flat plate experiments. PJ. Golden [62] developed a new dovetail fretting fixture to test aero-engine materials at room and elevated temperatures. The fixture had instrumentation for measurement of the normal contact force and the shear component of the contact force. A finite element model was developed to finalize the overall design of the system and to perform 
a contact analysis to obtain contact forces which were then compared to measured data. The contact analysis was conducted using Ti-6Al-4V material for specimens and pads at room temperature. This was done to simply the analysis as the titanium alloy is isotropic. The COF prescribed in the analysis was based on empirical test data, which varied from 0.3 to 0.7 [62].

The shear force $Q$ versus the normal contact force $P$ for a COF of 0.7 for the Ti-6Al-4V test is plotted in Fig. 47 (a). The solid line show the experimental data after the COF stabilized. The open squares represent the numerical results, where each square is one of 20 equally spaced time intervals for each of the three applied load steps of max, $\min$ and max. The dashed lines represent that boundary of the shear force for which $|Q| \leq \mu P$. When $|Q|=\mu P$, the dovetail specimen undergoes gross slip resulting in a sliding in or sliding out motion, depending on whether a max or min load was applied. Between the bounds, the resulting contact is in partial slip. The initial load ramp up occurred under gross slip conditions since the numerical results fell on top of the dashed lines, where $|Q|=\mu P$. This behavior was also noticed experimentally (not shown). After the initial gross slip, the dovetail specimen was wedged between the pads, representative of a blade disk dovetail attachment. When the load was reversed, the dovetail remained wedged in place and the contact changed from gross slip to partial slip. Fig. 47 (b) shows the pressure traction under the maximum applied force. As expected, the edges of contact have the highest pressure tractions. The mesh convergence study showed that the $25 \mu \mathrm{m}$ element size was sufficient in capturing the correct pressure distribution [62].
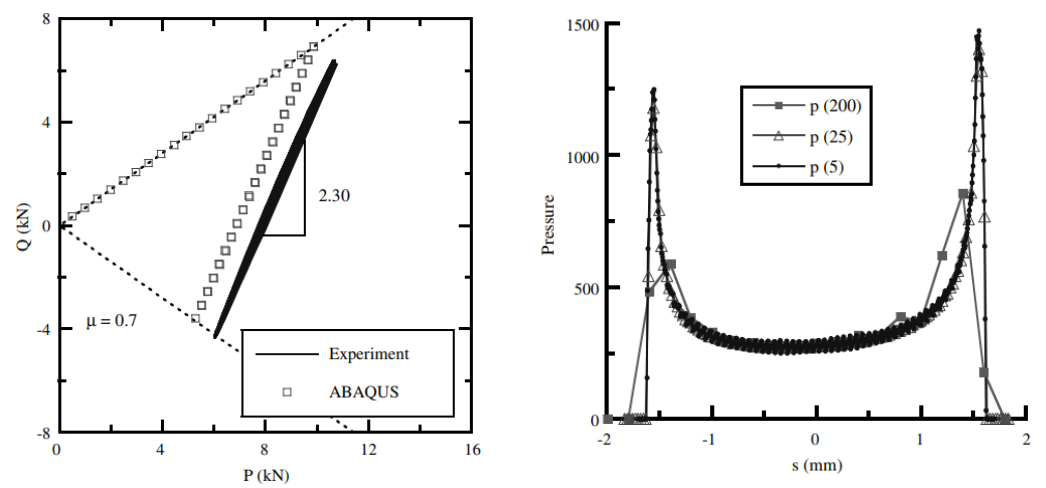

Fig. 47. (a) Shear versus normal contact force (b) Comparison of pressure tractions for various mesh sizes [62]

Several works [58], [63], [63], [64] use fracture mechanics based methodologies to assess dovetail fretting. These methodologies aim to model both the initiation and propagation phase of fretting fatigue. As such, several of these methodologies are used to determine whether fretting induced cracks will grow or arrest after a period of accelerated crack growth.

R. Rajasekaran et al. [58] used short crack arrest methods to determine the crack growth behavior for a biaxial fatigue experiment which simulated the loading of a dovetail blade attachment of aero-engines. The failed blade specimen is shown in Fig. 48 (a). Stress analysis was conducted to determine the bulk and bending stresses at the disk and blade. A finite element model was constructed for the dovetail blade attachment. The loading was applied in multiple steps with a friction coefficient of 0.1. Eight load steps were considered in total to ensure the shakedown of the non-linear history dependent tractions to a steady state. 
Short crack arrest methods were then used to predict whether vibratory loading would cause the nucleation and growth of a fretting crack. Since edges of contact have very large stress gradients, an effective approach to predict the behavior of a fretting crack would be to determine whether the growing crack arrests after growing in the short crack regime. This can be done using a stress intensity history along the crack depth in conjunction with a Kitagawa diagram. When stress intensities are larger than the threshold, crack growth will occur leading to failure, otherwise, crack arrest occurs. It was determined in this study that for higher friction coefficients, failure is more likely to occur as shown in Fig. 48 (b). The results show that when friction coefficient is 0.10 and lower, the stress intensity along the crack depth is lower than the El-Haddad limit, implying that crack arrest occurs. These predictions agreed well with the experimental results.
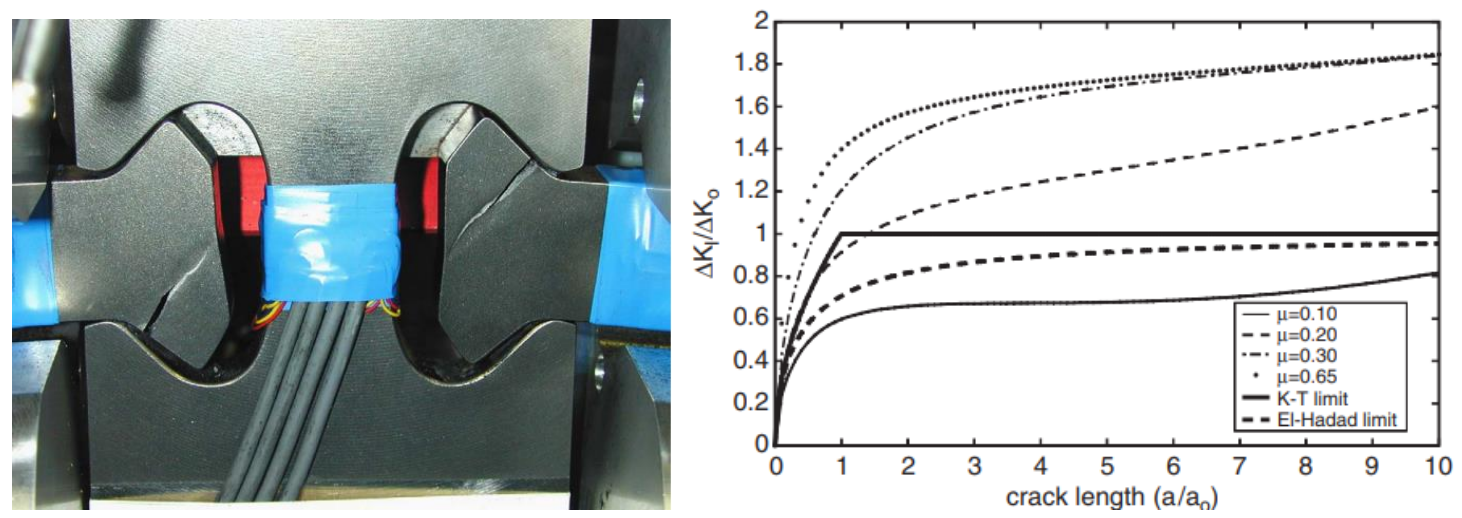

Fig. 48. (a) Failed blade specimens (b) Stress intensity along crack depth for various friction coefficients [58]

PJ. Golden et al. [63] [63] also use fracture mechanics based crack growth life methodology to predict fretting fatigue of experimentally tested $\mathrm{Ti}-6 \mathrm{Al}-4 \mathrm{~V}$ specimens. The specimens were tested with varying dovetail slot interface angles and were tested under various loading ratios. Contact stress software and finite element methods were used to determine the contact stresses and bulk stresses using a COF which varied between 0.16 and 0.34 based on experimental observations. Using the stresses obtained at the contact interface, life prediction for both nucleation and propagation were considered. For nucleation, an equivalent stress model was used to account for the multiaxial and mean stress effects. The equivalent stress was calculated at all points of interest [63].

The propagation life was predicted using Newman and Raju based models for semi elliptical cracks in tension and bending. The stress intensity factors were calculated at the edge of contact for which a fretting crack would nucleate. An initial crack length was assumed from which the crack propagation life was calculated. The results of the analysis showed that the total life of the component is dominated by propagation and is insensitive to the size of the initial crack due to the very high initial crack growth rates. It was also concluded that a potential exists in which crack growth would ultimately lead to crack arrest as the crack growth rate dies out due to a severe stress gradient at the local contact area. The final predicted initiation + propagation life was compared to experimental lives and was in good agreement [63]. PJ. Golden et al. [63] concluded based on this work that cracks initiate near edges of contact, whereas propagation and arrest is controlled the bulk far field loading.

A fretting fatigue map response was developed by S. Fouvry et al. [64] shown in Fig. 49 to formalize frettingfatigue damage with respect to both crack nucleation and crack propagation. A procedure was introduced to estimate 
the two damage thresholds. For nucleation, high cycle fatigue criterion which considers stress gradient and size effects was used. For crack propagation, a short crack arrest criteria is used which utilizes Kitagawa and El Haddad models.

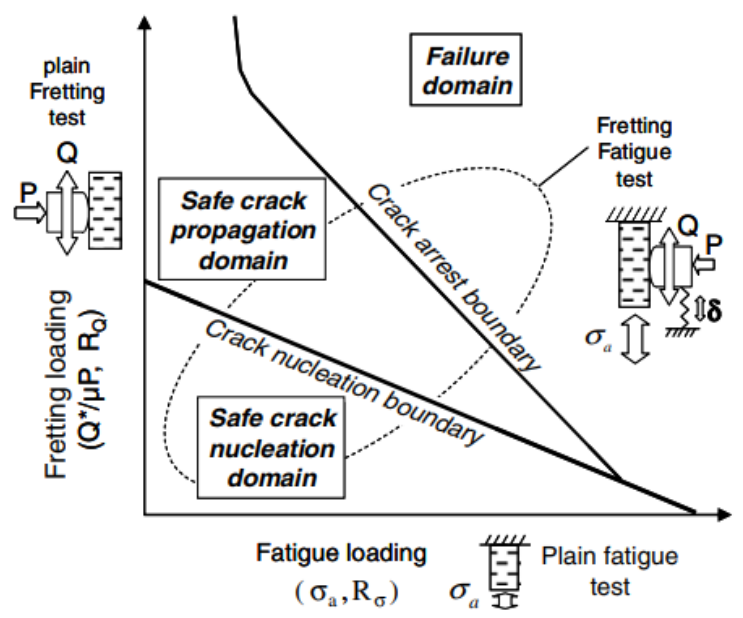

Fig. 49. Fretting map concept [64]

The fretting map shows three evolutions of cracks. The first is referred to as the safe crack nucleation domain, and occurs when loading is below threshold fretting fatigue conditions and as such, no cracks are nucleated. The second is a safe crack propagation domain, for which loads which are above threshold resulting in crack nucleation but then later crack arrest occurs due to very sharp stress gradients which die out into the depth of the crack. The final zone is the failure domain and occurs when the bulk or contact loading is further increased causing the propagation and failure of nucleated fretting cracks [64].

This study used elastic material properties along with the averaging approach. It is explained that this approximation was used since in the high cycle fatigue regime, it is widely understood that plasticity has minor effects. Further to this, elastic responses typically overestimate stresses yielding more conservative fatigue parameters. Also, very small partial slip conditions are imposed in thus study, resulting in very narrow hysteresis responses which may be approximated through elastic conditions [64].

Recent efforts have been made by Fracture Analysis Consultants Inc [18] to develop a numerical methodology which is capable of modeling fretting crack nucleation and discrete crack growth using FRANC3D software. FRANC3D leverages powerful finite element analysis tools such as ANSYS to solve for the complex three dimension stress states in sliding contact, and has been widely used to simulate three dimensional crack growth in complex loading. FRANC3D software has built in fretting fatigue prediction models such as the aforementioned Ruiz-Chen, critical shear stress, SWT, and modified SWT parameters. The recently developed tool allows for users to select one of several fretting nucleation models to accurately predict fretting crack nucleation cycles including the location and orientation of the initial fretting induced crack. In addition to this, there is an option available to use to compute the fretting parameters based on the averaging method developed by Araujo et al. [25]

FRANC3D has been applied to simple fretting pad experiment applications and to the more complex fan slot dovetail fretting problem shown in Fig. 50. In both simulations, the software was able to successfully calibrate the location for which the fretting induced crack nucleates, and the number of cycles required to nucleate [18]. 

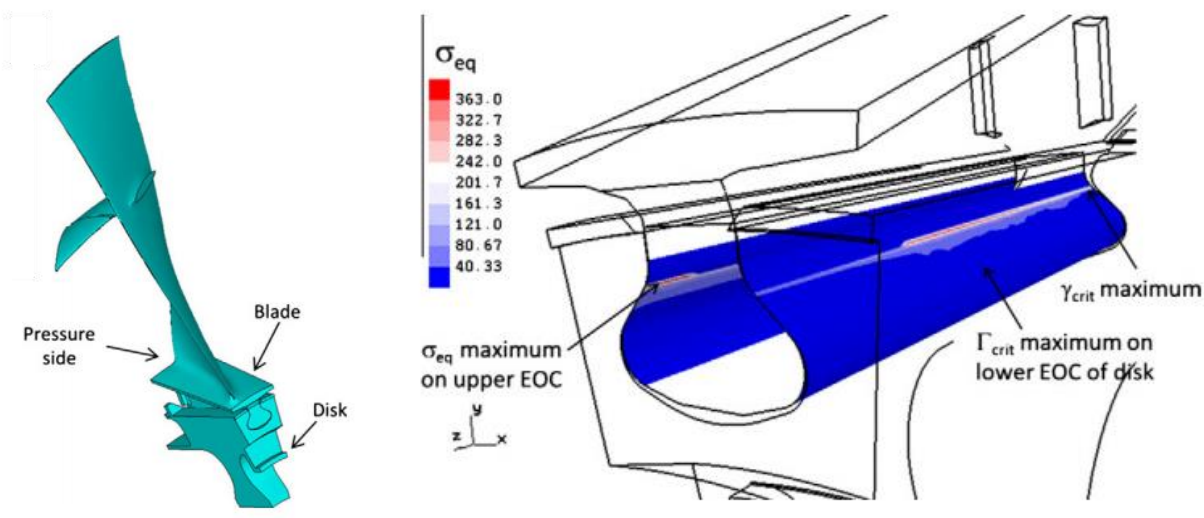

Fig. 50. Fretting nucleation parameter for dovetail fan attachment [18]

Following nucleation, FRANC3D has capabilities to perform 3D crack growth simulations and to determine crack propagation life using an outputted stress intensity history as shown in Fig. 51. In fretting fatigue, this crack growth is divided into two stages, the initial stage is inclined growth from the contact surface at edge of contact driven by the large shear stresses. The second and final stage exhibits crack growth perpendicular to the contact surface due to the applied bulk tensile stress [18].
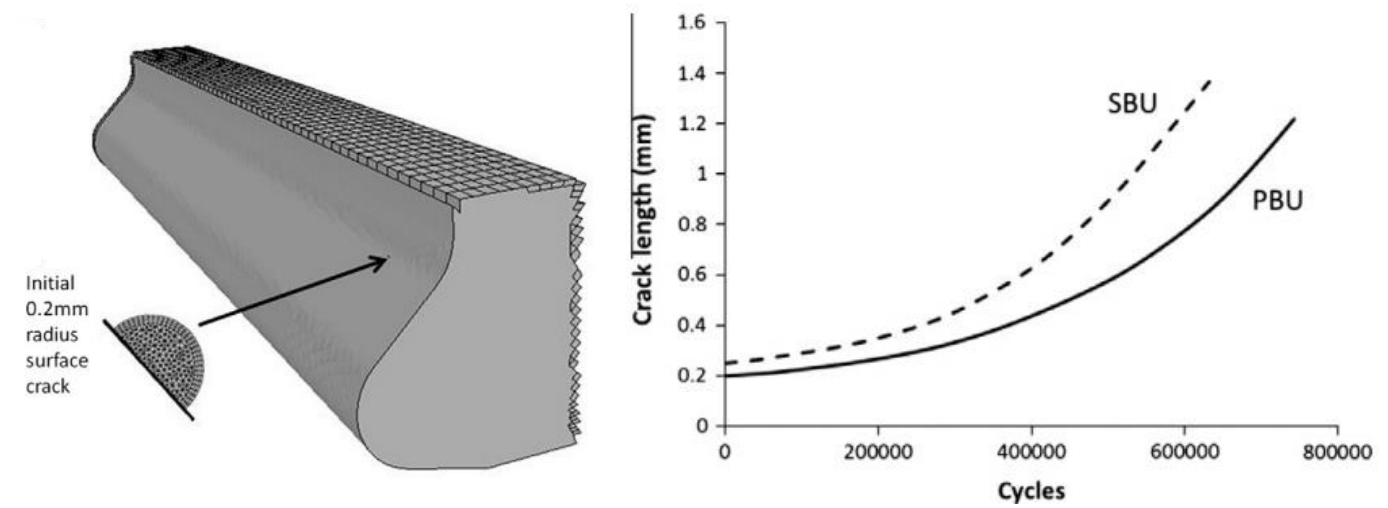

Fig. 51. Discrete crack growth analysis [18]

Barlow et al. [8] utilized FRANC3D to simulate the 3D fretting fatigue crack growth of an aircraft engine fan blade attachment using the max tensile stress theory. The simulation was done considering centrifugal and aerodynamic loads, with a prescribed coefficient of friction along the contact surface. The stress intensity factors combined with the FNK and aircraft spectrum loading were used to determine the crack propagation life. At the time of this work, FRANC3D did not have built in fretting fatigue prediction models and as such, the crack was inserted at the highest principal stress location. Now, fretting fatigue parameters such as the modified SWT which considers effects of wear may be employed to insert the crack at the maximum calculated parameter, which does not necessarily correspond to the most highly stressed location. The results from the simulation correlated well with field results as shown in Fig. 52 [8]. 


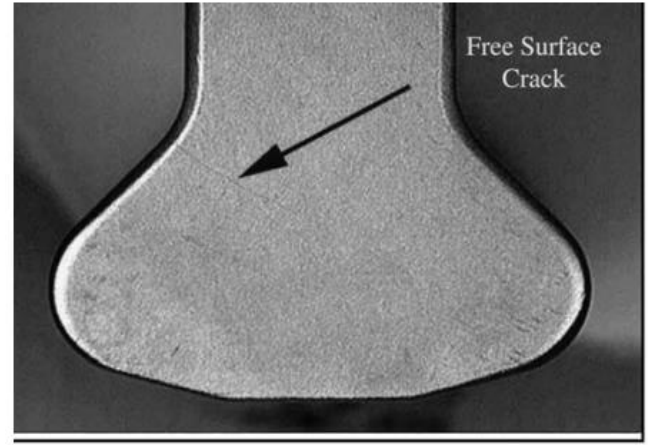

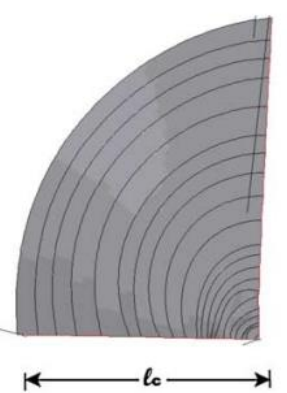

(a) Top View

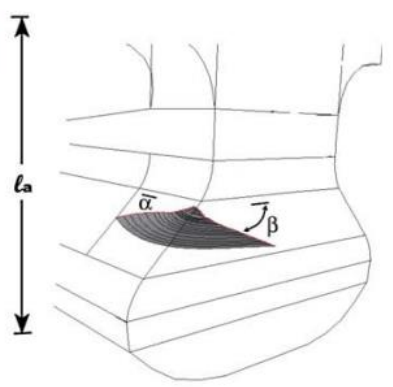

(b) ISO View

Fig. 52. Free surface crack on dovetail attachment fan blade and simulated crack growth [8]

Older versions of the software, FRANC2D, which are capable of two dimensional crack growth, have also been used by Fadag et al. [65]. In this work, a finite element sub-model method shown in Fig. $\mathbf{5 3}$ was used to predict the crack propagation life of titanium alloy, Ti-6Al-4V specimens for a cylinder-on-flat and flat-on-flat geometry. The numerical-experimental approach was used to determine the crack initiation lives. It was found that $90 \%$ of the total fretting fatigue life of the specimens was spent in the crack nucleation phase in the HCF regime. The effects of contact load, coefficient of friction, and tangential force to the behavior of crack growth was also studied. It was found that by increasing these mentioned parameters, that the crack propagation life was decreased.

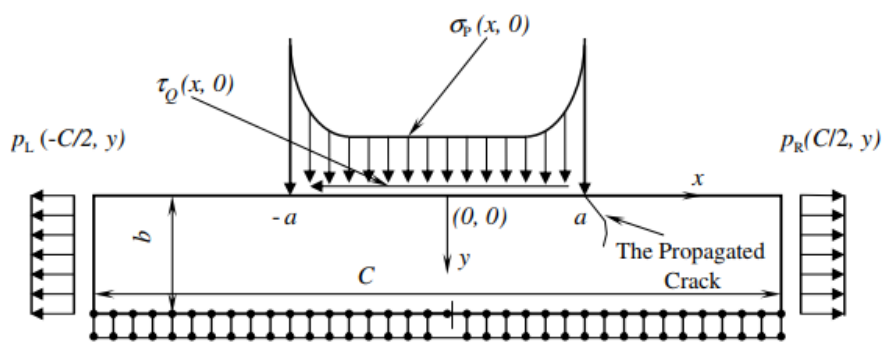

Fig. 53. Sub-model used in FRANC2D [65]

FRANC2D was used to perform discrete crack growth analysis using linear elastic fracture mechanics principles. A crack was inserted at the trailing edge of the contact surface since experimental studies showed that the crack in fretting fatigue tests initiated at or near the trailing edge with an angle of 45 degrees to the contact surface. It was found that the crack propagation life was independent to the initial crack length when it was larger than $50 \mu \mathrm{m}$ [65]. 


\section{FATIGUE FAILURE OF AN IBR}

This chapter presents a 3D crack growth simulation of an aircraft engine high pressure compressor blade which fractured in service. Using FRANC3D, an initial crack is inserted and grown using the max tensile stress theory. A numerical method was developed to capture the stress field under LCF and HCF loading by superimposing test measured dynamic loading with steady stresses. In doing so, the predicted crack trajectory, aspect ratio, and shape more closely agreed with the fractured airfoils compared to performing the simulation under LCF loads alone. This supports findings from fractographic investigation that the crack exhibits HCF rather than LCF characteristics.

\subsection{FRACTOGRAPHIC INVESTIGATIONS OF FRACTURED COMPRESSOR BLADES}

When climbing through 3,700 meters an engine oil pressure red-light illuminated, followed by vibration and the inter turbine temperature reading spiking full scale. The pilot shut down the engine, declared an emergency, and returned to the departure airport. Disassembly of the engine found a fracture on one of the high pressure compressor rotor stages. Fractographic investigations were conducted for the fractured compressor blades for three similar occurrences in the field to determine root cause. The compressor blade in question is made of a titanium alloy Ti6Al-4V, roughly measures $53.3 \mathrm{~mm}$ in chord length with a maximum airfoil thickness of $4.3 \mathrm{~mm}$ as shown in Fig. 54 . The final fracture surfaces for the three separate field events are shown in Fig. 55.

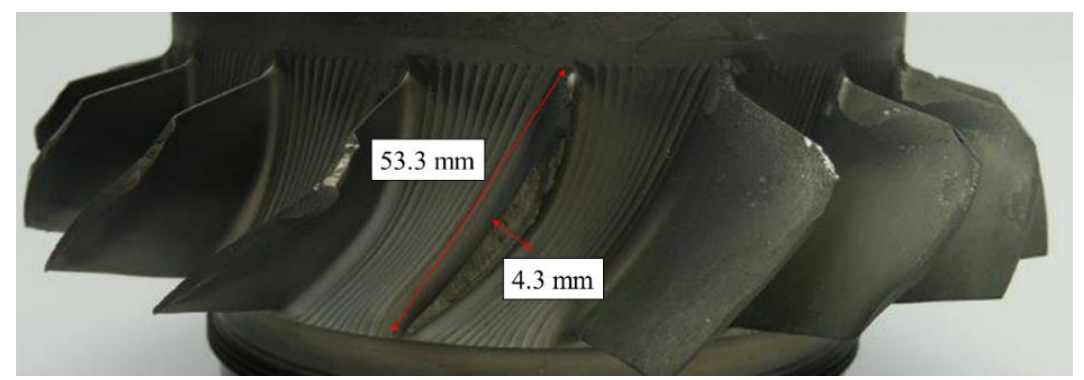

Fig. 54. Macro view of a fractured high pressure compressor airfoil, $53.3 \mathrm{~mm}$ chord length, $4.3 \mathrm{~mm}$ thickness
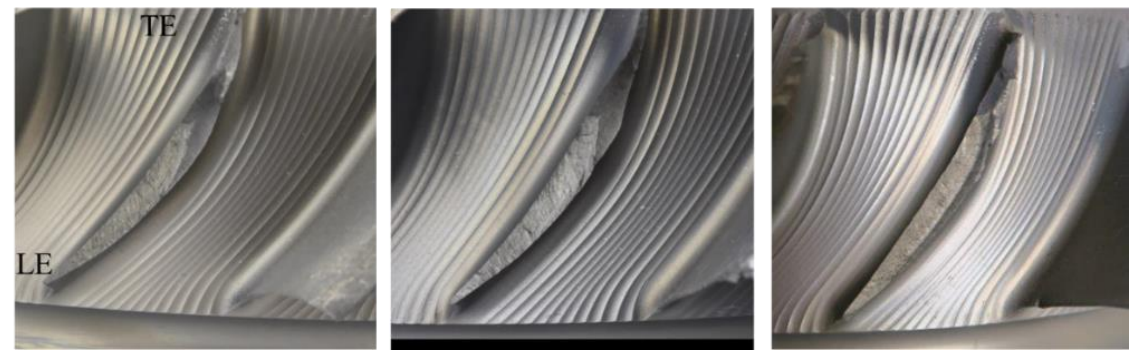

Fig. 55. Airfoil fracture surfaces of a high pressure compressor airfoil for events A, B, C from left to right

Laboratory investigations indicate that as the fatigue crack progressed from the initiation site at the airfoil pressure side to the suction side, it became gradually larger along the chord, with a bias towards the leading edge (LE). After the crack reached a critical depth, growth became unstable which led to the compressor blade releasing from the rotor due to ductile overload failure and forming a shear lip. Fractographic investigations found for all fracture events, the crack initiated near the mid chord on the pressure side of the airfoil as shown Fig. $\mathbf{5 6}$ for event C. The river lines 
indicated by the yellow arrows radiate from the crack origin indicated by the red arrow. The crack progression marks (beach marks) indicated by the green arrows are most evident near the trailing edge (TE) and LE locations.

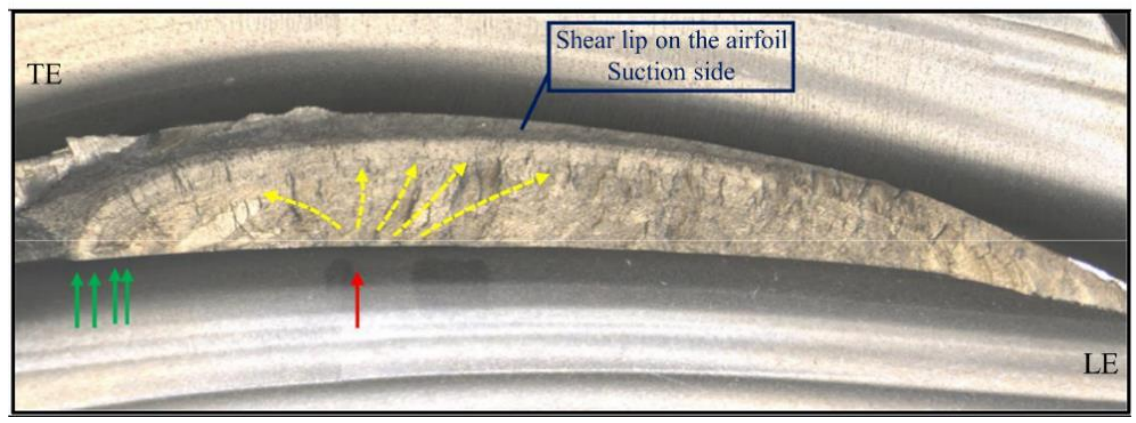

Fig. 56. Fracture surface showing crack origin (red), river lines (yellow), and beach marks (green) - Event C

EDS analysis and SEM examination confirmed that the major elements and microstructure of the material at the crack initiation site were in acceptable proportions as shown in Fig. 57, and thus material anomaly was ruled out as the cause of fracture.
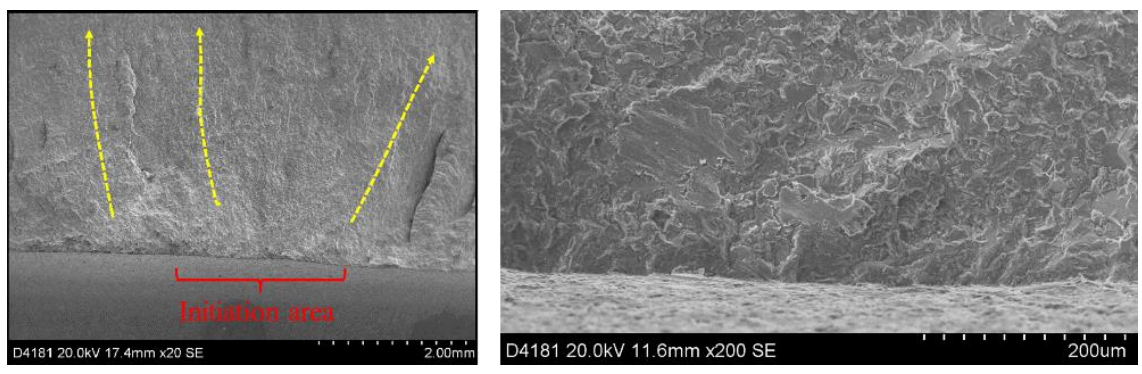

Fig. 57. Crack initiation site. No anomalies were found through SEM and EDS - Event C

SEM was used to identify progression marks which could indicate changes to the crack propagation. It was concluded that the marks were not associated with LCF crack growth but rather attributed to HCF given that continuous river lines crossed several of these progression marks at both the leading and trailing edge locations shown in Fig. 58. Attempts were made to measure striation spacing for the fractured airfoil events using SEM. However, the number of field incidents which presented visible striations was too small to allow for the striation count with a certain confidence interval. Ultimately, the HCF striations were too fine to accurately resolve, and did not follow a decay with depth as do LCF striations, supporting conclusions of Hershko et al [66] and Cherolis [67] .
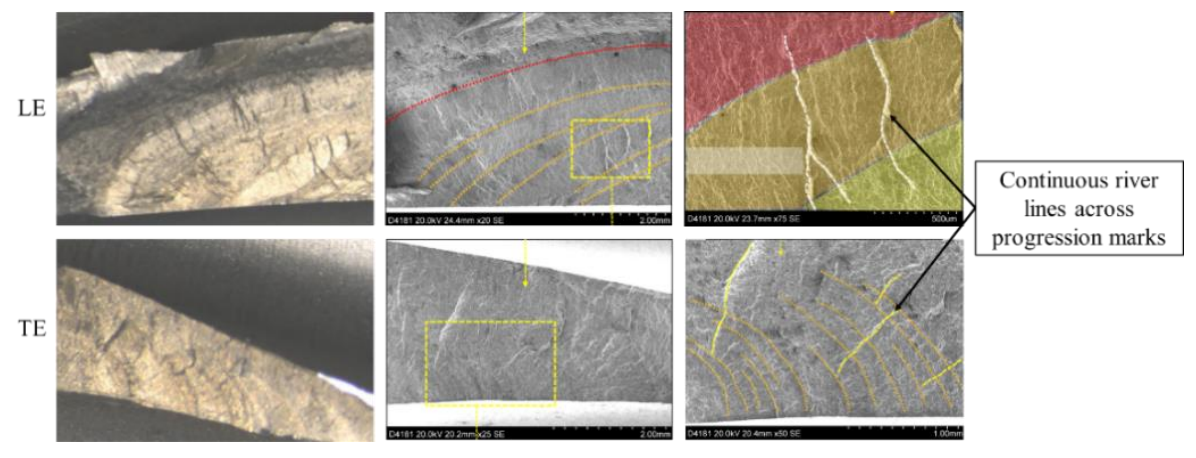

Fig. 58. SEM showing river lines cross multiple progression marks at TE \& LE, characteristic of HCF - Event C 


\subsection{FINITE ELEMENT MODELLING}

\subsubsection{STATIC ANALYSIS}

Simulating the crack growth required a 3D ANSYS finite element model for the pristine compressor rotor. A static structural analysis was conducted to determine the steady stresses on the airfoil subjected to an angular velocity and temperature gradient corresponding to resonance and maximum takeoff conditions. Linear elastic material properties were used in the analysis. The finite element model results for the maximum takeoff conditions are presented in Fig. 59 (a). For temperatures corresponding to these flight conditions, the tensile properties of a representative Ti-6Al-4V alloy are $\nu=0.34, \mathrm{E}=104 \mathrm{GPa}$, and $\sigma_{\mathrm{y}}=630 \mathrm{MPa}$. The FE results indicate a peak steady stress near the mid chord at the pressure side of the airfoil corresponding to the crack initiation location. At this location, there is an equivalent of 45,000 Newtons of force pulling radially outward per square centimeter. From this, $3 \%$ is caused by thermal stresses due to a temperature gradient of 60 degrees Celsius acting across a distance of 28 $\mathrm{mm}$ between the peak steady stress location and the hotter tip of the airfoil. The remaining $97 \%$ of load is dominated by centripetal forces due to angular velocities close to 30,000 revolutions per minute (RPM). The peak equivalent stress was compared to the Ramberg-Osgood stress-strain curve for the corresponding temperature to check for levels of plasticity. This was done to validate the use of a linear elastic material. Fig. 59 (b) shows the operating Von-Mises stress lies well within the elastic portion of the stress strain curve.

(a)

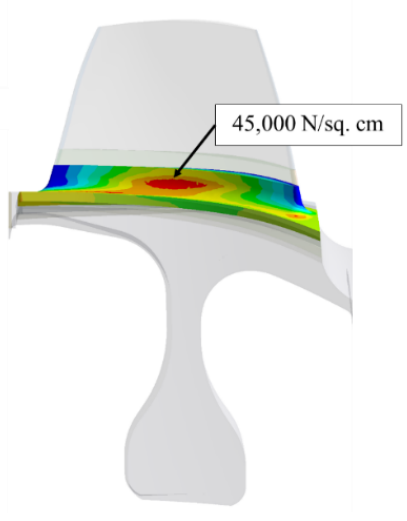

(b)

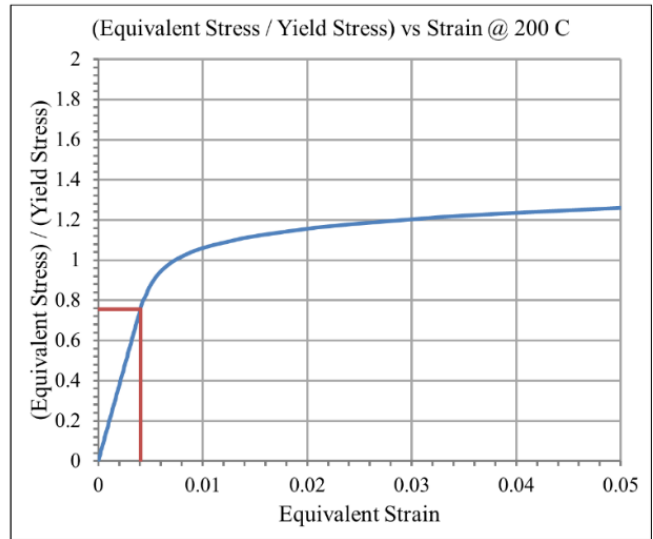

Fig. 59. (a) Max principal stress near airfoil release at maximum takeoff conditions (b) Stress strain curve

\subsubsection{MODAL ANALYSIS}

Since fractographic investigations suggested that the growth was characteristic of HCF, a modal analysis was conducted on the pristine compressor rotor using ANSYS to obtain the airfoil's dynamic mode shapes, and to determine its associated scaled vibratory stress and deflections. Fig. 60 shows a single dominant bending mode of the compressor blade which was believed to be the main culprit leading to the final failure of the airfoil. As the blade bends towards the suction side in Fig. 60 (a), the resulting stress at the pressure side is tensile which tends to open the crack. Conversely, as the blade bends towards the pressure side in Fig. 60 (b), the resulting stress at the pressure side is compressive which closes the crack. The principal stresses for the dominant bending mode is shown in Fig. 61. 

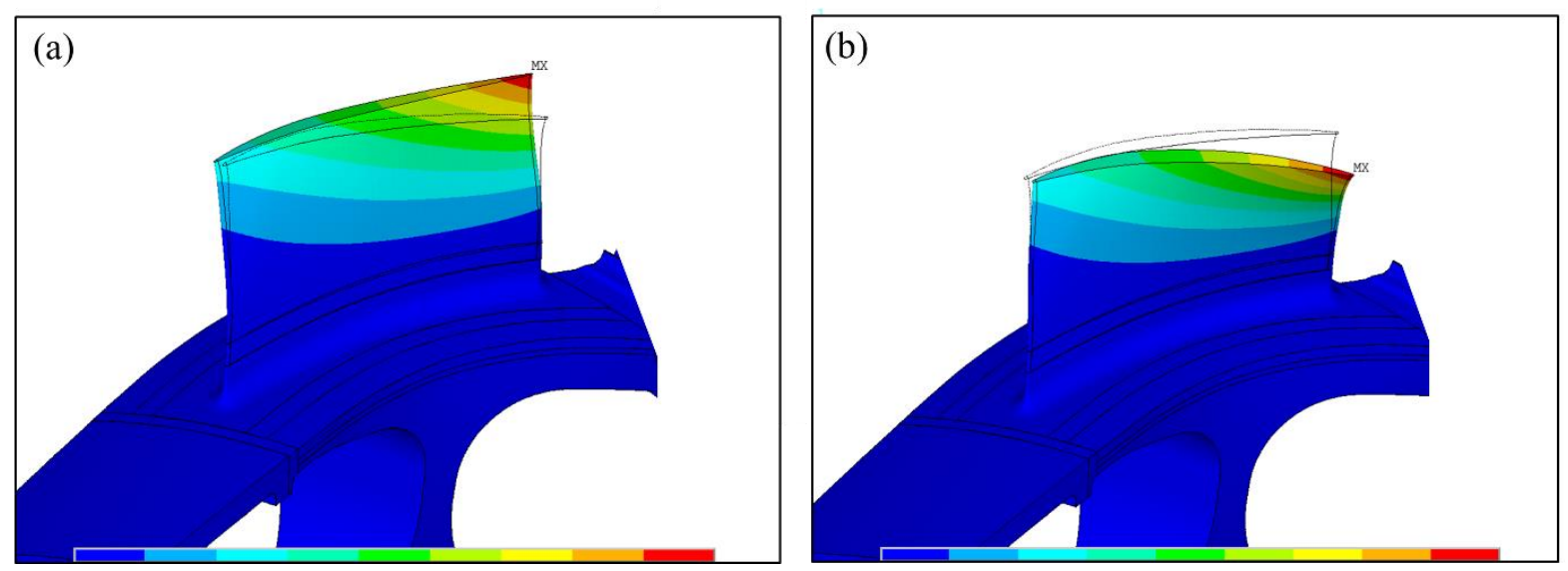

Fig. 60. Blade deflection for dominant bending mode (a) tension on pressure side (b) tension on suction side

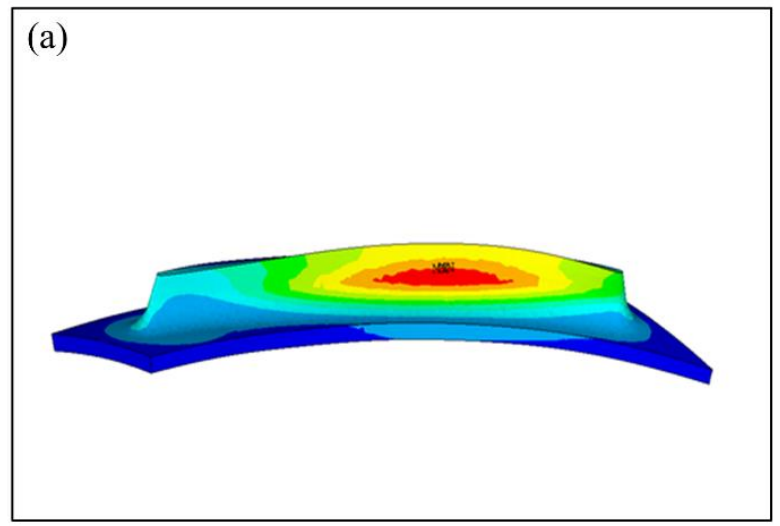

(b)

Fig. 61. Resulting blade root max principal (a) tension on pressure side (b) tension on suction side

Solutions obtained through ANSYS modal analysis result in scaled deflections and stresses for determined mode shapes. The actual magnitude of the vibratory stresses and deflections depend on the magnitude of excitation which can be obtained by means of strain gauge testing or Non-Intrusive Stress Measurement Systems (NSMS) respectively. In the latter, fiber optics are used to direct a light source such as a laser into the path of the rotating blade from which a deflection is measured. Dynamic stresses are then calculated at the location of interest using stress to deflection ratios from ANSYS modal results in conjunction with NSMS measured tip deflections. In this study, the leading edge tip deflections were provided at resonance speed across various operating conditions using laser probes aligned at the leading edge tip. Fig. 62 shows the resulting scatter of test results measured in percentage of airfoil span. Later, a method was implemented to convert the modal analysis into a static structural analysis using the measured test data. 

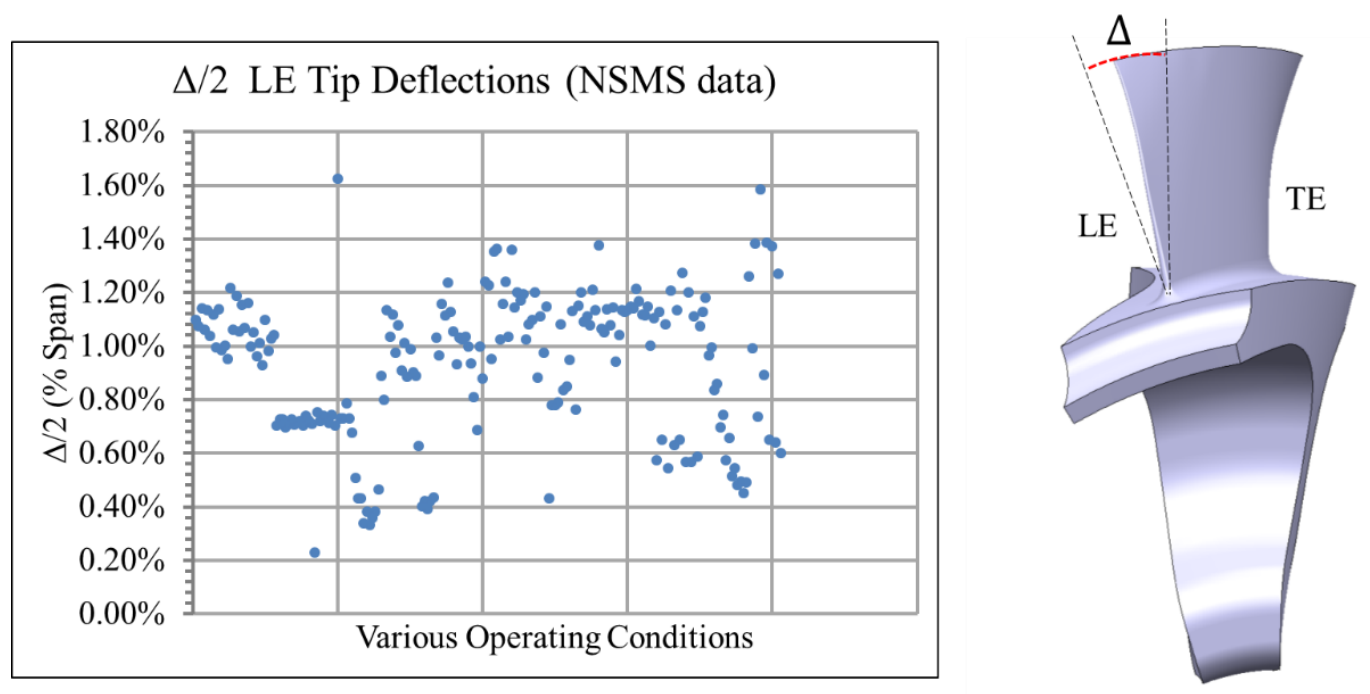

Fig. 62. NSMS measured tip deflections at leading edge in percent span for various operating conditions

\subsubsection{COMBINING STATIC + MODAL ANALYSIS}

The peak steady stress location determined from the static analysis and peak dynamic stress location determined from the modal analysis are shown on the airfoil root in Fig. 63. From the three recorded failures, two cracks initiated near the peak dynamic stress location and one initiated near the peak steady stress location.

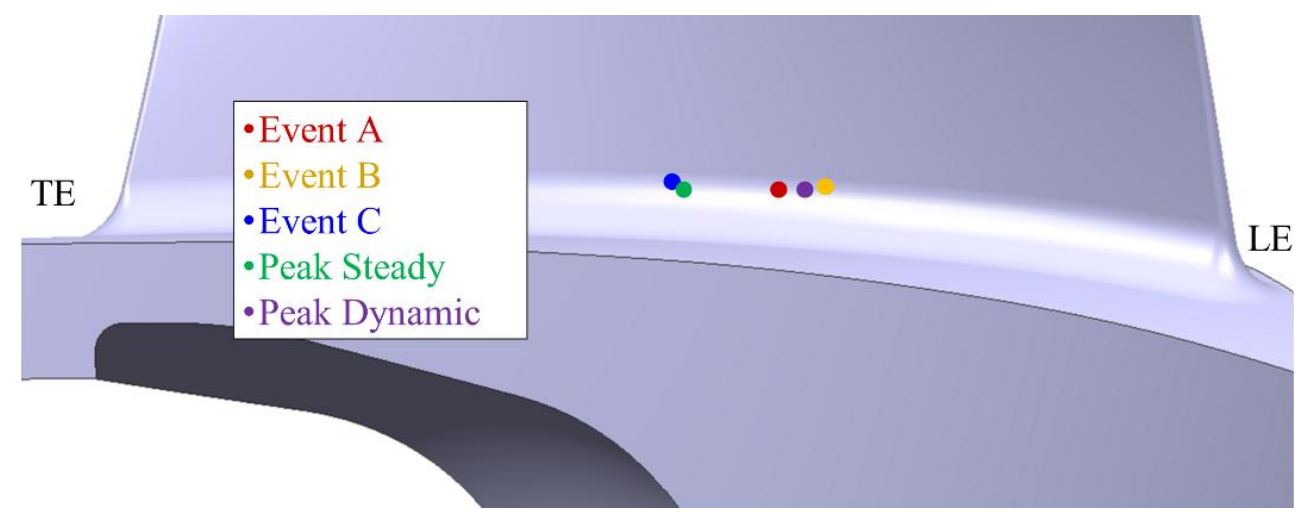

Fig. 63. FE results for peak steady and dynamic stress locations and crack locations of events A, B, and C

Simulating the crack growth under combined HCF+LCF loading requires a method of superimposing the modal results to those of the initial static structural solution. This was done using a concentrated constant bending load added to the tip of the blade as shown in Fig. 64 (a) which resulted in stress and deflection fields closely resembling those obtained through modal analysis for the single dominant bending mode of interest. The magnitude and vector direction of the applied bending force was optimized until the finite element model produced a tip deflection equivalent to the data collected by the NSMS laser probes. Fig. 64 (b) and Fig. 64 (c) show the final max principal stress distribution obtained after several iterations to the magnitude and direction of the force. The stress distribution obtained through the tip bending force matched very closely with the distribution obtained through the modal analysis shown in Fig. 61. Furthermore, the resulting magnitude of the alternating leading edge tip deflection from the static structural 
analysis was found to be $1.3 \%$ of the span. This agrees well with the higher end of the NSMS measured data shown in Fig. 62. The final combined steady and dynamic model consisted of two load steps with reversed tip bending dynamic forces to simulate HCF loading superimposed on the steady stresses from centripetal and thermal loads. The Von-Mises effective stresses at the crack location under combined HCF+LCF loading fell in the elastic regime of the Ramberg-Osgood curve. Thus, the use of linear elastic material properties was considered sufficient for future crack growth simulations. This conclusion was also validated through an FE analysis of the cracked blade making use of a Ramberg-Osgood model for the material. The crack opening stresses $\sigma_{y y}$ directly ahead of the crack tip were calculated based on the elastic K-field singularity and compared to the stresses obtained from the FE solution. The resulting stress distributions were characteristic of small scale yielding given that outside of the plastic zone, the elastic $\mathrm{K}$ field singularity resulted in a stress state which matched very closely to that obtained from the elastic-plastic FE solution. Furthermore, the radius of the plastic zone calculated for plane strain conditions was small (7\%) in comparison to the size of the crack.
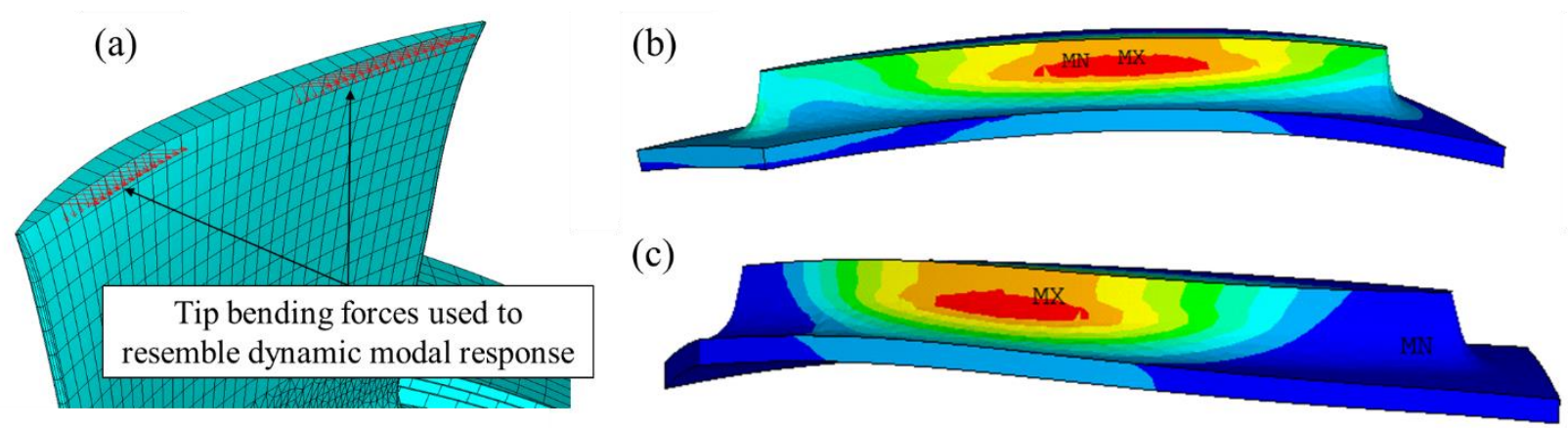

Fig. 64. (a) Tip bending force and max principal stress (b) tension at pressure side (c) tension at suction side

\subsection{CRACK GROWTH SIMULATION}

The crack growth simulation was modeled for two loading scenarios. Scenario 1) with pure LCF loading and 2) with combined HCF+LCF loading. For both simulations, the MTS method was used to predict the direction of crack growth. MTS predicts that a crack will propagate at an angle $\theta$ which results in the maximum principal stress governed by equation (100). This is achieved by growing in a path in which $\Delta \mathrm{K}_{\mathrm{II}}=0$, where $\Delta \mathrm{K}$ is dependent on the loading spectrum. Equation (101) represents $\Delta \mathrm{K}$ for pure LCF loading where $\mathrm{K}_{\min }$ corresponding to engine shut down is zero. Equation (102) represents $\Delta \mathrm{K}$ for combined $\mathrm{LCF}+\mathrm{HCF}$ non-proportional loading where $\mathrm{K}_{\min }$ is a result of compressive dynamic stresses at the pressure side of the airfoil and $\mathrm{K}_{\max }$ is a result of tensile dynamic stresses which drive the opening of the crack. FRANC3D accounts for the non-proportionality in the crack growth loading schedule through the 'Non-Proportional Cyclic' option [48]. When the 'Non-Proportional Cyclic' option is selected, FRANC3D prompts the user to identify two unique finite element load steps corresponding to $\mathrm{K}_{\max }$ and $\mathrm{K}_{\min }$ in which the direction of principal stress vectors local to the crack change during loading. These two load steps are used for the computation of $\Delta \mathrm{K}_{\mathrm{I}}, \Delta \mathrm{K}_{\mathrm{II}}$, and the resulting crack turning angle $\theta$ for each crack front advancement.

$$
\Delta \sigma_{\theta \theta}=\frac{1}{\sqrt{2 \pi r}} \cos \left(\frac{\theta}{2}\right)\left[\Delta K_{I} \cos ^{2}\left(\frac{\theta}{2}\right)-\frac{3}{2} \Delta K_{I I} \sin \theta\right]
$$




$$
\begin{gathered}
\Delta \mathrm{K}(\mathrm{LCF})=\mathrm{K}_{\max }(\mathrm{LCF})-\mathrm{K}_{\min }=\mathrm{K}_{\max }(\mathrm{LCF}) \\
\Delta \mathrm{K}(\mathrm{LCF}+\mathrm{HCF})=\mathrm{K}_{\max }(\mathrm{LCF}+\mathrm{HCF} \text { Tension })-\mathrm{K}_{\min }(\mathrm{LCF}+\mathrm{HCF} \text { Compression })
\end{gathered}
$$

An initial circular penny shaped surface crack with radius of $100 \mu \mathrm{m}$ and aspect ratio of 1 was inserted parallel to the engine axis at the peak steady stress location and analyzed under pure LCF loading, and at the peak dynamic stress location and analyzed under combined HCF+LCF loading. The initial crack size was chosen to match with the earliest laboratory striation count, corresponding to a crack depth of $100 \mu \mathrm{m}$. For both simulations, the crack was grown until reaching a final depth or surface length.

A Kitagawa diagram was constructed using equation (103) to determine whether the chosen initial flaw size was beyond the critical flaw size so that long crack da/dn vs $\Delta \mathrm{K}$ data could be used. The long crack threshold SIF and the material endurance limit used for the diagram correspond to the temperature and loading ratio under resonant vibration conditions. The $\triangle \mathrm{K}(\mathrm{LCF}+\mathrm{HCF})$ term is obtained using equation (102) by subtracting $\mathrm{K}_{\min }$ from $\mathrm{K}_{\max }$, both of which are computed by FRANC3D at each crack growth advancement.

$$
\Delta \sigma_{t h}=\frac{\Delta K_{t h}}{Y \sqrt{\pi a}}=\Delta K_{t h} * \frac{\Delta \sigma(L C F+H C F)}{\Delta K(L C F+H C F)}
$$

As per the Kitagawa diagram, the transition from short crack behavior to long crack behavior where the use of fracture mechanics is valid occurs at a flaw depth size of about $50 \mu \mathrm{m}$. This is half the size of the initial crack in the simulation and is consistent with data collected for titanium alloys which shows cracks with a depth below $50 \mu \mathrm{m}$ is characterized by short or small crack behavior [68].

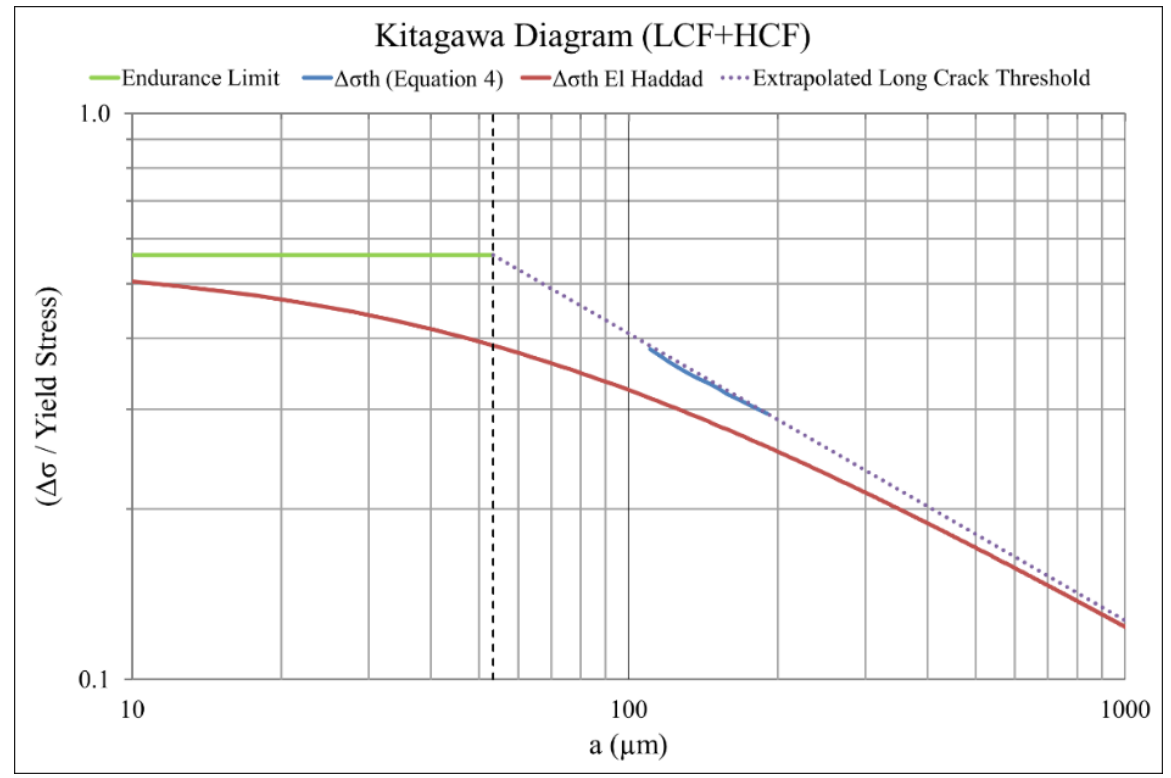

Fig. 65. Kitagawa diagram under LCF + HCF Loading 


\subsubsection{PURE LCF LOADING}

Under pure LCF loading, the direction, aspect ratio, and shape predicted by the crack growth simulation shown in Fig. 66 did not agree with the fractured airfoils. In the absence of dynamic loading due to resonant vibration, the crack grew in a direction perpendicular to the maximum principal stress, which in the case of pure LCF loading, is dominated by only the radial stress produced by the centripetal force.

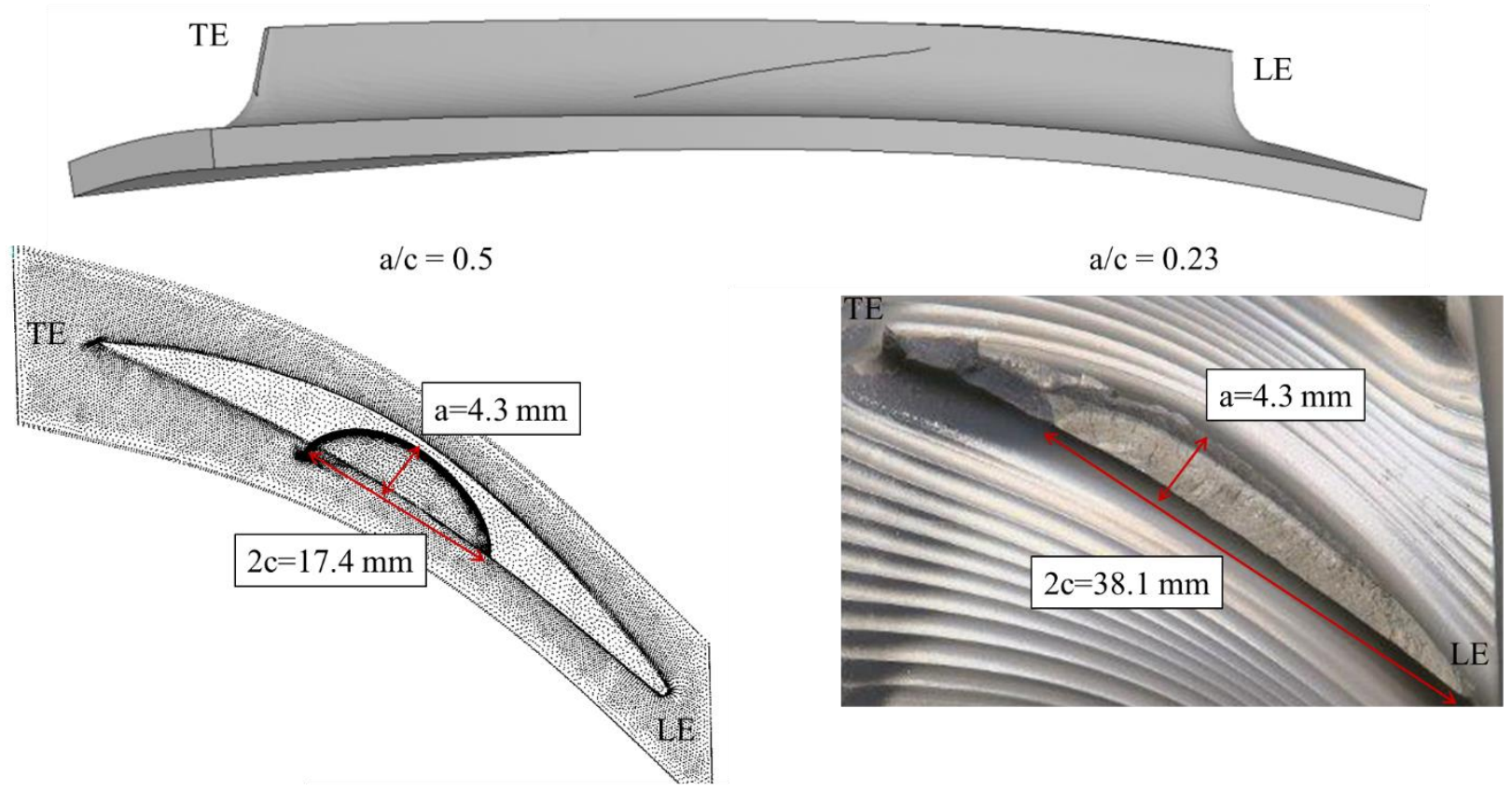

Fig. 66. Crack growth simulation under pure LCF loading

Fig. 67 shows the mode I and mode II SIF's along the crack front for the first 55 crack iterations computed by FRANC3D. As the crack grew, the mode I SIF's increased in magnitude but held a similar shape along the crack front. The maximum mode I SIF's occur near the crack end points closer to the free surface and the minimum SIF's occur at maximum crack depth. For the initial crack, the mode II SIF along the crack front was non-zero. There was a kink from the initial crack orientation to the next, and the remaining crack iterations grew in a path to satisfy MTS theory, resulting in a value of $\Delta \mathrm{K}_{\mathrm{II}}=0$. The final crack predicted through the simulation under pure LCF loading had an aspect ratio of 0.5 . This implied that the crack gradually became larger along the chord due to the fact that the crack ends closer to the free surface have larger mode I stress intensities relative to the remaining crack front. The fractured component had an aspect ratio of 0.23 , suggesting that the crack end points in the actual loading condition had a much larger $\Delta \mathrm{K}$ relative to the remaining crack front. It is also noted that the simulation did not capture the biased crack growth towards the leading edge since the mode I SIF's at both ends of the crack front are equal in magnitude, resulting in equal crack growth towards both leading and trailing edges of the airfoil. 

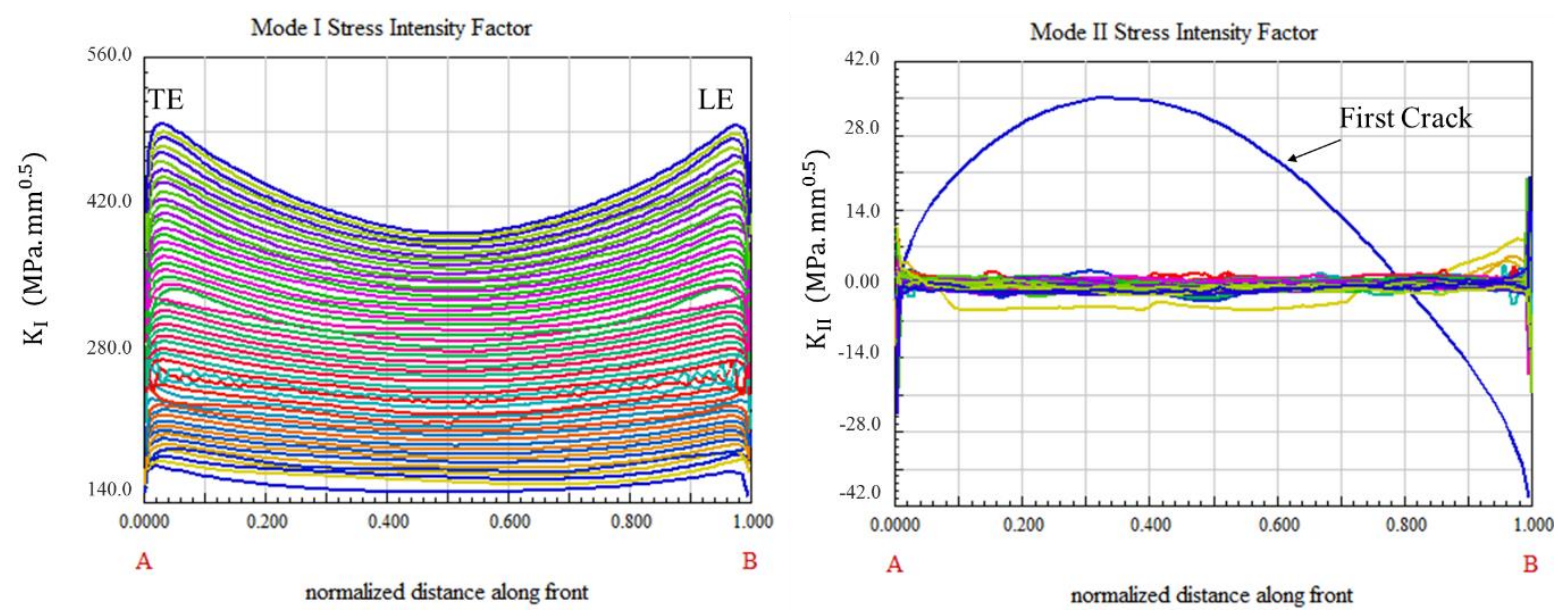

Fig. 67. Mode I and II stress intensities along first 55 crack fronts under pure LCF loading $(A=T E, B=L E)$

\subsubsection{COMBINED HCF + LCF LOADING}

Under combined HCF+LCF non-proportional cyclic loading, the direction, aspect ratio, and shape predicted by the crack growth simulation shown in Fig. $\mathbf{6 8}$ agreed well with the fractured airfoils, indicating that the fractures are due to combined LCF and HCF loading. The concentrated bending force added to simulate the HCF resonant vibration resulted in a direction of crack growth which better aligned with the fractured components. This supports the initial assumption that the single first bending mode was responsible for the crack growth and ultimately led to fracture.

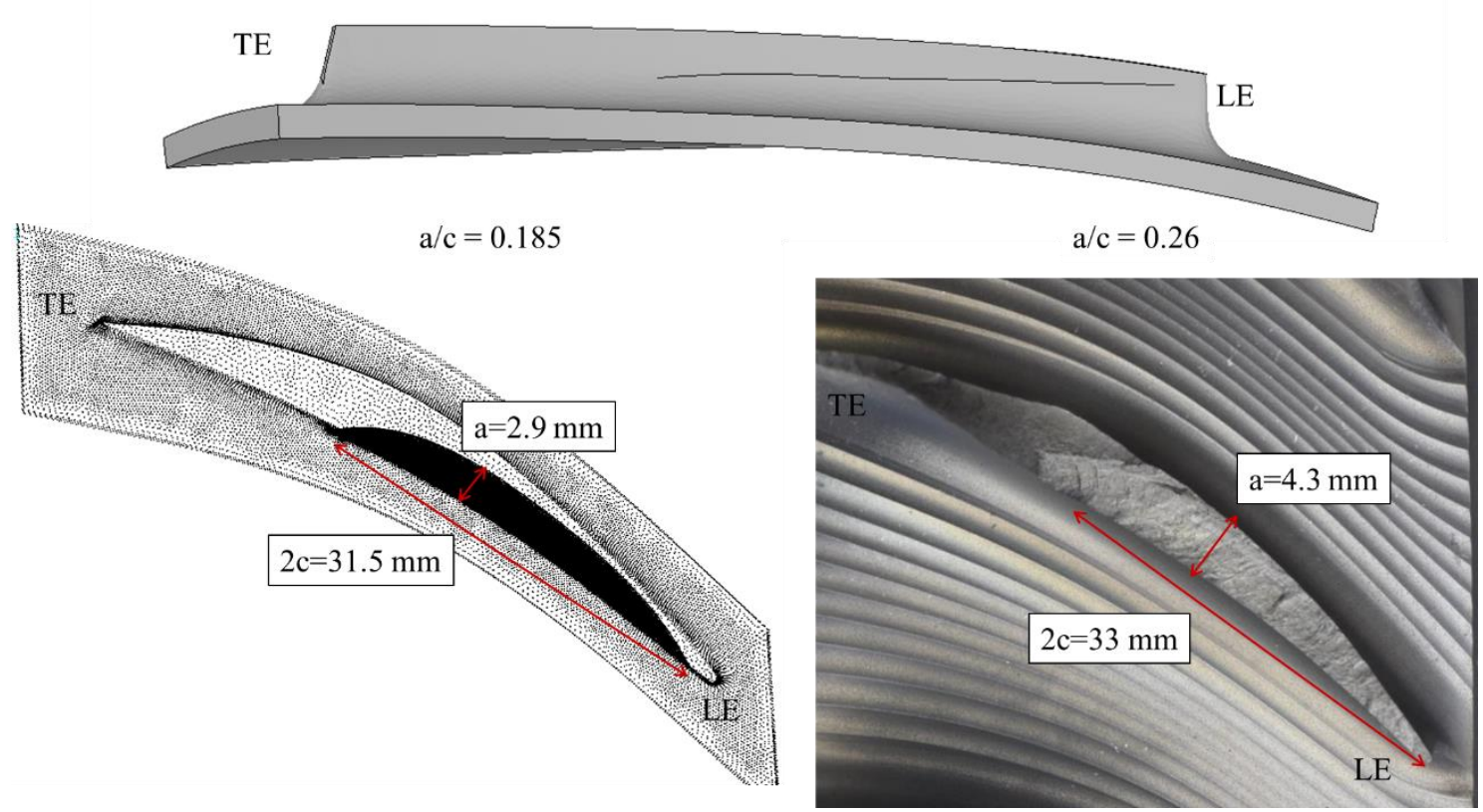

Fig. 68. Crack growth simulation under combined HCF + LCF loading

The mode I and mode II SIF's along the crack front for the first 55 crack iterations computed by FRANC3D are shown in Fig. 69. The max load case represents LCF+HCF in tension and the min load case represents LCF+HCF in compression. As the crack grew, the mode I stress intensities increased in magnitude but held similar shapes. For the max load case, the tension due to resonant bending further opened the crack, resulting in a higher mode I SIF than 
those under pure LCF loading, with an absolute peak near the trailing edge. For the min load case, the compression due to resonant bending tended to close the crack, resulting in a linearly decreasing mode I SIF's along the crack front. The absolute peak occurred near the trailing edge and gradually decreased to a minimum at the crack front end points closest to the leading edge. Two factors contributed to the non-symmetry in the stress intensities along the crack front for the max and min load cases. The first is a consequence of the applied concentrated tip load resulting in a slight twist of the airfoil and the second is a consequence of the vibratory bending stress distribution which linearly decreases along crack depth from its maximum value at the pressure side.

The crack was grown using MTS with $\Delta \mathrm{K}$ given by equation (102) where $\Delta \mathrm{K}_{\mathrm{I}}$ and $\Delta \mathrm{K}_{\mathrm{II}}$ are obtained by subtracting the stress intensities for the two load cases $\left(\mathrm{K}_{\max }\right.$ and $\left.\mathrm{K}_{\min }\right)$. Thus, the mode II stress intensities shown in Fig. 69 show that the crack correctly grew in path resulting in $\Delta \mathrm{K}_{\mathrm{II}}=0$, with minimal kinking from the initial crack orientation. The final crack predicted through the simulation had an aspect ratio of 0.185 . The accelerated crack growth along the chord is a direct consequence of the cyclic compression and tension. By subtracting the mode I SIF for the min load case from the max load case, the crack end points closer to the free surface had a much larger mode I SIF compared to the remaining crack front due to higher vibratory bending stresses. It is also noted that by incorporating the dynamic loading through a concentrated constant bending force, FRANC3D accurately predicted the biased crack growth at the leading edge which was characteristic of all the recorded failure events due to a larger $\Delta \mathrm{K}_{\mathrm{I}}$ at the leading edge compared to the trailing edge. This becomes especially apparent at later stages of the simulation as the crack becomes large in size as shown in Fig. 70, where $\Delta \mathrm{K}$ is simply obtained by subtracting $\mathrm{K}_{\min }$ from $\mathrm{K}_{\max }$ along the crack front. 

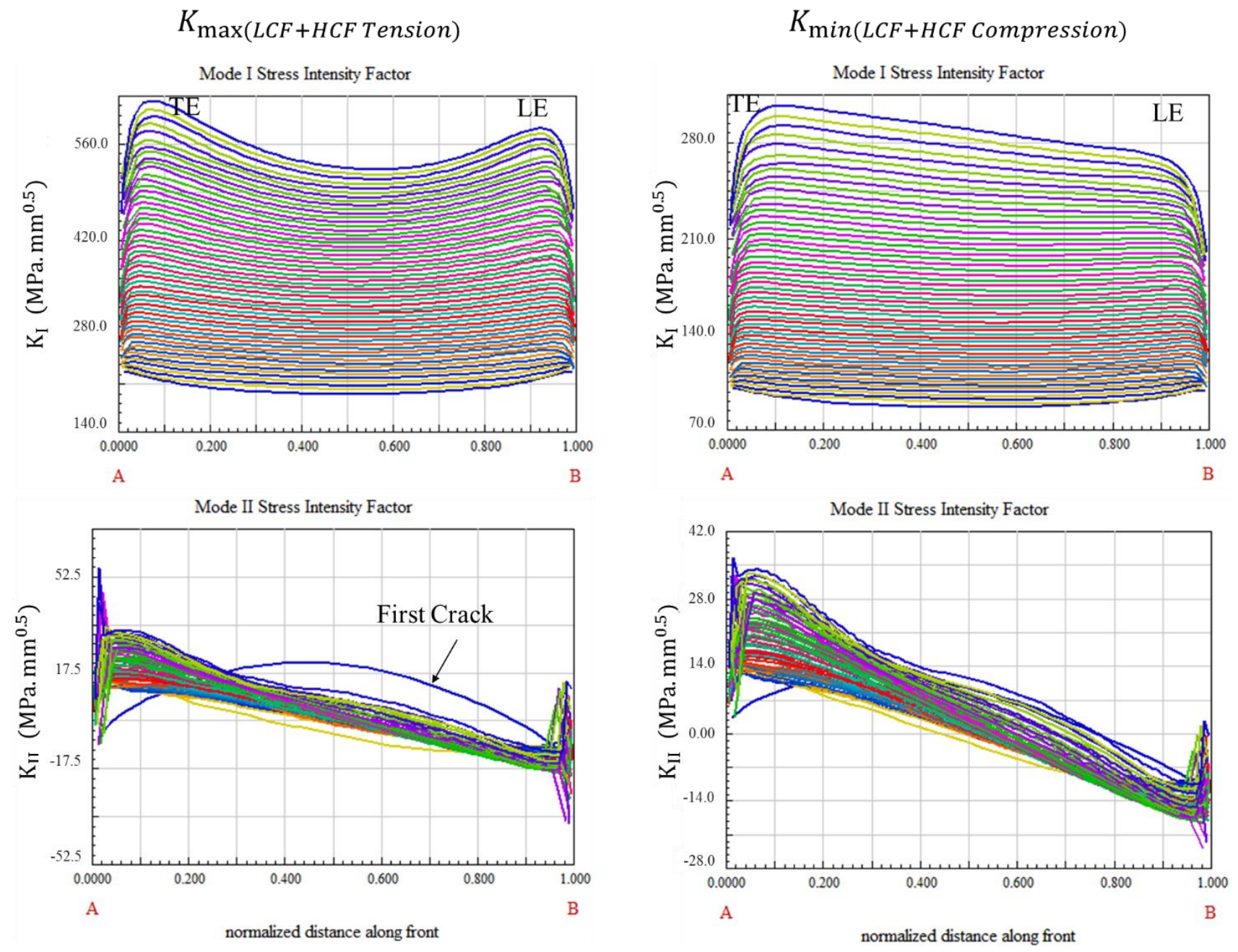

Fig. 69. Mode I and II stress intensities along first 55 crack fronts under LCF \& HCF Loading $(A=T E, B=L E)$

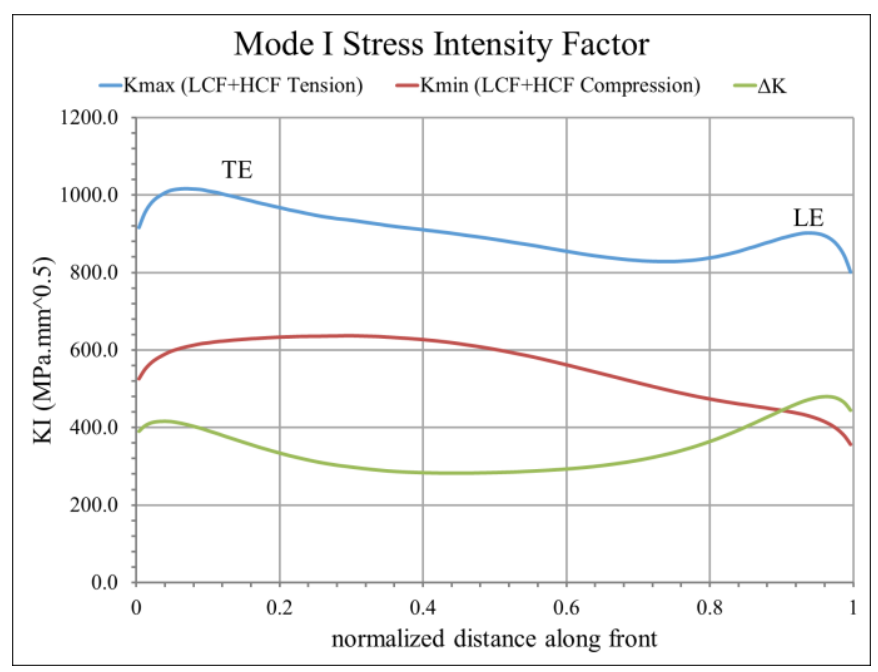

Fig. 70. - Mode I stress intensities along crack front corresponding to crack depth of $2.5 \mathrm{~mm}$ 


\subsection{CRACK PROPAGATION LIFE FOR THE LCF + HCF SIMULATION}

Byrne et al. [6] identified two regimes of crack growth, a LCF crack growth regime and a combined HCF+LCF crack growth regime. Hawkyard et al. [69] used a linear summation model to compute the combined crack growth rate in a flight block consisting of steady stress LCF cycles, and multiple HCF cycles achieved at a unique resonance speed induced by aerodynamic excitations or mechanical vibration. It is noted that the combination of the initial crack size used in the simulation and the magnitude of the dynamic stresses resulted in a SIF greater than the threshold stress intensity range. As such, the initial crack modeled in the simulation had already entered the HCF+LCF crack growth regime in which dynamic stresses actively contribute to the advancement of the crack.

The FNK [47] model was used to determine the crack propagation rate under combined HCF + LCF loading. The FNK model accounts for the retardation near threshold, acceleration near fast fracture and crack closure effects.

$$
\begin{gathered}
\frac{d a}{d N}(B L O C K)=\frac{d a}{d N}(L C F)+\sum \frac{d a}{d N}(H C F+L C F) \\
\frac{d a}{d N}=\frac{C(1-f)^{n} \Delta K^{n}\left(1-\frac{\Delta K_{t h}}{\Delta K}\right)^{p}}{(1-R)^{n}\left(1-\frac{\Delta K}{(1-R) K_{c}}\right)^{q}}
\end{gathered}
$$

The $\mathrm{C}, \mathrm{n}, \mathrm{p}$, and, $\mathrm{q}$ are empirical constants derived by curve fitting test data at varying environmental conditions such as temperature and varying load ratios. $\Delta \mathrm{K}$ is the applied stress intensity factor range along a path of all crack front advancements,$\Delta \mathrm{Kth}$ is the threshold stress intensity range which is dependent on loading ratio, and $\mathrm{K}_{\mathrm{c}}$ is the critical stress intensity factor. The $\mathrm{R}$ and $\mathrm{f}$ represent the load ratio and crack closure function respectively. The fatigue crack growth properties used in this simulation are an outcome of exhaustive company specimen testing and are left out intentionally for confidentiality. However, taking $\mathrm{C}=4.466 \mathrm{e}-13$ (da/dN in mm/cycle, $\Delta \mathrm{K}$ in $\mathrm{MPa} \sqrt{\mathrm{mm}}$ ), $\mathrm{n}=3.2$, $\mathrm{p}=0.25$, and $\mathrm{q}=0.75$ to be the crack growth properties for Ti-6Al-4V from the FRANC3D material database results in a close approximation of the crack growth rate behavior in comparison to company retrieved material data.

Fig. 71 depicts an LCF - HCF flight block of a typical mission for the compressor rotor of interest. By ignoring intermediate conditions, the flight mission consists of one major LCF cycle of 'Start-Takeoff-Shutdown' and one minor LCF cycle of 'Descent-Thrust Reverse-Taxi (Idle)'. In addition to the LCF cycles, there is an accumulation of many HCF cycles when a pilot transiently passes through resonance speeds across various flight conditions. These are referred to in the figure as 'transient pass-through' (TP). The accumulation of HCF cycles, and thus, the predicted crack growth life is very sensitive to the time it takes for an engine to transiently pass through resonance speeds and the dynamic frequency of the excited compressor blade. Due to the transient nature of dynamic loading, it is very difficult to estimate the actual number of HCF cycles accumulated per flight. Therefore, in this study, a sensitivity analysis was done to determine the final crack propagation life by varying the number of HCF cycles which the compressor rotor may see in operation per flight as it transiently passes through resonance speeds. 


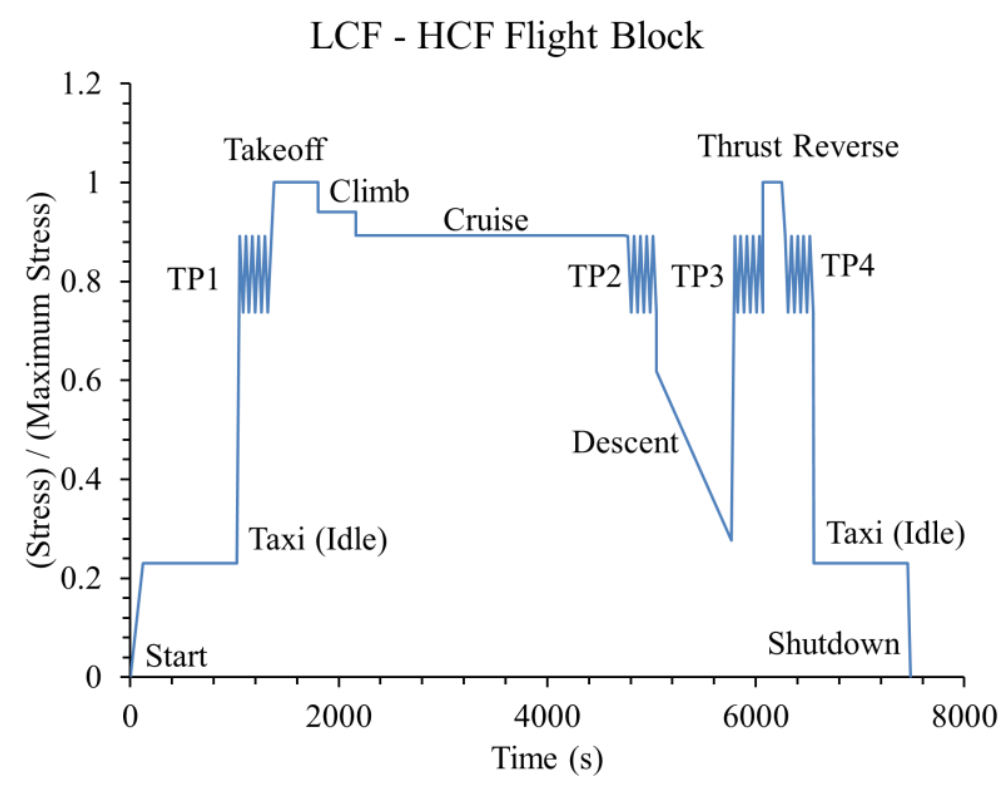

Fig. 71. LCF - HCF flight block showing a typical mission of the compressor rotor

From the crack growth simulation, M-Integral stress intensities calculated by FRANC3D along the crack depth were used as shown in Fig. 72 in conjunction with the LCF - HCF flight block and long crack da/dN data to predict crack growth lives. For LCF cycles, $\Delta \mathrm{K}$ is obtained through equation (101), where $\mathrm{K}_{\max }$ corresponds to the stress intensities under centripetal and thermal loading for LCF major and minor loading conditions. For HCF cycles, $\Delta \mathrm{K}$ is obtained through (102) by subtracting $\mathrm{K}_{\min }$ which corresponds to compressive dynamic bending stresses at the pressure side from $K_{\max }$ which corresponds to tensile dynamic bending stresses at the pressure side both of which are superimposed on the LCF steady stresses at resonance conditions. It is noted that although HCF dynamic stresses and stress intensities are lower in magnitude compared to the major or minor LCF cycles, their effect on the block crack growth rate is amplified depending on the number of accumulated cycles.
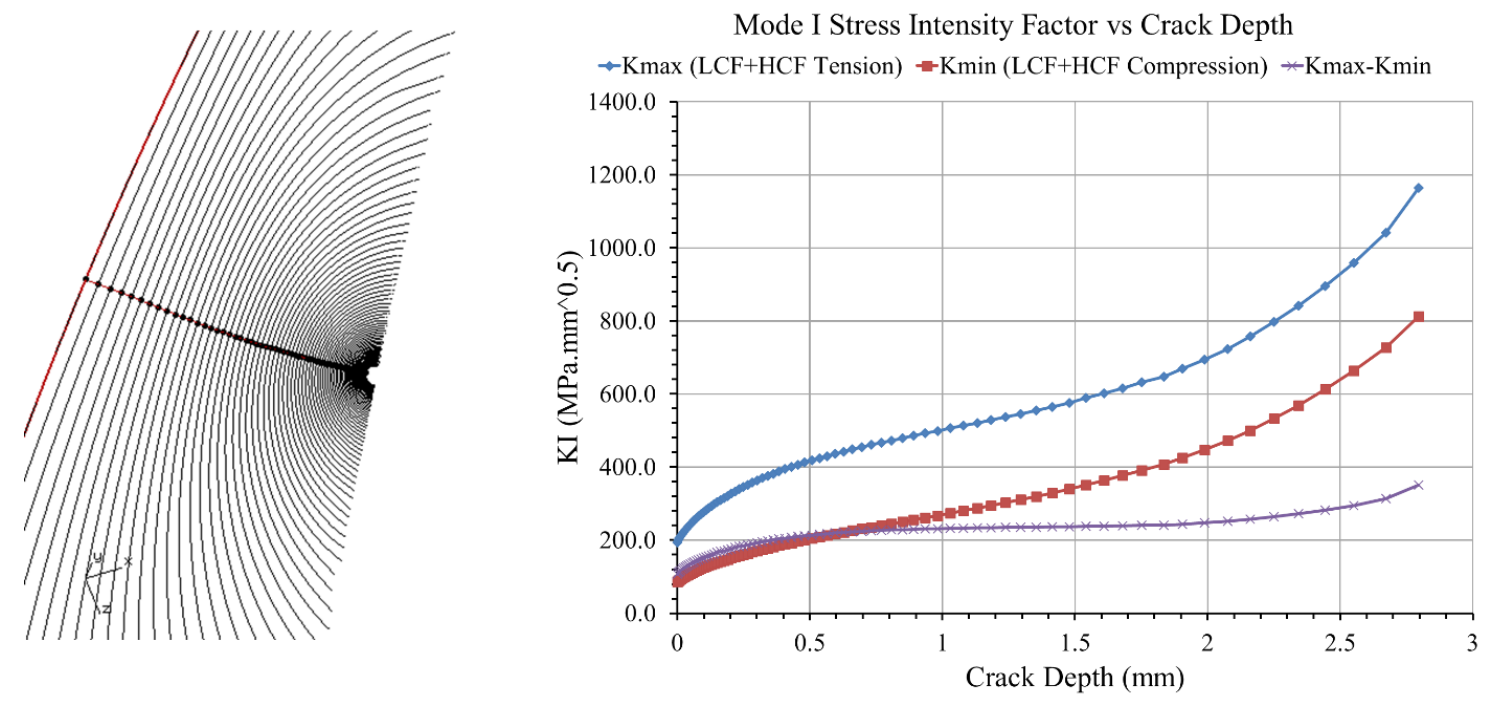

Fig. 72. Mode I SIF along crack depth under LCF+HCF Tension and LCF+HCF Compression 
Fig. 73 (a) shows the determined crack propagation lives measured in flights calculated with respect to number of assumed HCF cycles per flight. The effects of the LCF cycles on the crack growth was studied by performing the calculation twice, once with LCF contribution, and once without LCF contribution. It is concluded by this exercise that the effect of the major and minor LCF cycles on the final crack growth life diminishes as the number of HCF cycles per flight increases. For instance, at $50 \mathrm{HCF}$ cycles per flight, accounting for the LCF minor and major cycle's results in a crack growth life of 4,900 flights versus 5,180 flights when effects of LCF are ignored. For 100 HCF cycles per flight, the crack growth life is dominated purely by HCF loading, resulting in 2,500 flights to failure, whether or not the effects of LCF were taken into account. It is noted however that the LCF loads contribute to the overall crack growth behavior by imposing a positive mean stress to the HCF cycles, resulting in higher crack growth rates as determined through the FNK model.

It is important to note that these results are a direct outcome of the linear summation model employed through equation (104), which does not account for potential load history effects resulting from LCF+HCF loading. Byrne [6], showed through experimental testing that corner notch specimens made of Ti-6Al-4V exposed to LCF overloads reduce the influence of HCF cycles on the block crack growth rates and also increase the magnitude of SIF at which HCF becomes active. The study went on to show that for extreme overload conditions, the effects of HCF to overall crack growth is negligible. Conversely, corner notch specimens made of Ti-6Al-4V when tested at typical loading conditions [69] have shown that the linear summation model gives satisfactory crack propagation life predictions under major and minor cycle loading. Linear summation has also been used successfully by [70], [71], [72], and [73] for similar load applications. Given that the compressor rotor in this study was subjected to typical loading conditions and not overload conditions, the use of the linear summation model was considered sufficient.

For the three compressor blades in this study, the averaged final initiation + propagation life was 7,000 flights. Based on Fig. 73 (a), it may be concluded that the rotor accumulated a minimum of 35 HCF cycles per flight. However it is very difficult to accurately predict or measure an average number of HCF cycles accumulated on a per flight basis. Thus, a more meaningful approach would be to determine the crack propagation life with respect to number of HCF cycles to failure. Fig. $\mathbf{7 3}$ (b) shows the total number of HCF cycles to failure which is calculated by multiplying the final crack growth lives from Fig. $\mathbf{7 3}$ (a) with the number of assumed transient HCF cycles per flight. Based on the linear summation model, it was noticed that when the number of HCF cycles per flight is low, most of the crack growth is attributed to LCF loading, meaning that less HCF cycles and more LCF cycles are accumulated before blade fracture. As the number of HCF cycles per flight continues to increase, the effect of LCF becomes negligible and the total number of HCF cycles to failure asymptotically approaches 255,000 cycles. 

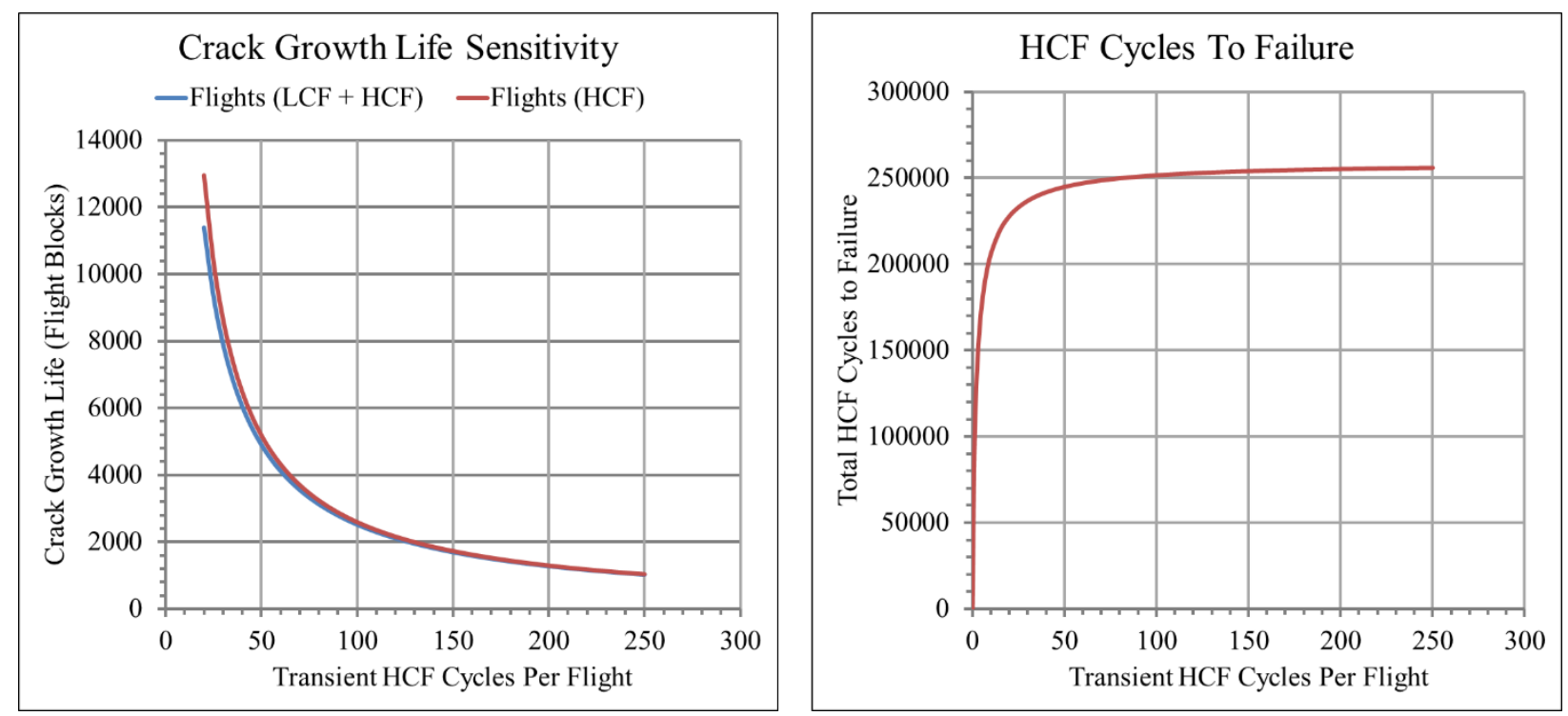

Fig. 73. (a) Crack growth life sensitivity to transient HCF cycle accumulation per flight (b) HCF cycles to failure

\subsection{FINDINGS}

1. Fractographic investigation found that airfoil fracture surface exhibits HCF loading characteristics.

2. Combined LCF+HCF cyclic loading was simulated in a static structural analysis by superimposing LCF stresses due to centripetal and thermal loads with a concentrated bending force at the tip of the blade which resulted in a stress and deformation distribution equivalent to the solution obtained through modal analysis for the airfoils dominant bending mode. This corresponds to the suspected mode which led to the failure of the components. The magnitude and vector direction of the bending force was optimized in the static structural analysis until the resulting tip deflection matched the test measured data.

3. Under LCF loading only, the direction, aspect ratio, and shape predicted by the crack growth simulation did not agree with the fractured airfoils. Only after capturing the entire LCF + HCF stress field did the predicted crack trajectory and shape agree with the fractured airfoils. This supports findings from fractographic investigations that the crack grew under HCF loading.

4. Results from the simulation indicate that the dominant bending mode obtained from the modal analysis is in fact the mode shape which caused the final fracture of the components.

5. HCF striations were too fine to resolve and did not follow a decay with depth as do LCF striations.

6. Based on the linear summation model, the effects of the LCF major and minor cycles to overall crack growth is less significant compared to the contribution of HCF loading.

7. Although HCF is the primary mechanism of fracture, LCF loading accelerates crack growth by imposing a positive mean stress to the alternating HCF dynamic stresses, resulting in higher crack growth rates as determined through the FNK model.

8. The converged crack propagation life of the compressor airfoil was found to be 255,000 HCF cycles. 


\section{FATIGUE FAILURE OF A DOVETAIL BLADED FAN}

The fretting fatigue and crack propagation of an aircraft engine fan dovetail attachment made of Ti-6Al-4V alloy has been numerically modeled and compared with controlled spin test results. The study suggests the fretting-specific modified SWT parameter predicts the nucleation location of the crack more accurately compared to the plain fatigue SWT parameter. The fretting model uses a prescribed coefficient of friction value of 0.7 , obtained through calibration of 3D crack growth simulations with the fractured components in regards to crack trajectory, aspect ratio, shape, and propagation life.

\subsection{SPIN TESTING AND FRACTOGRAPHIC INVESTIGATIONS}

In this study, three separate dovetail fan assemblies Fig. 74 (a) were tested in controlled spin pit environments for a defined speed range of 2000 to 11000 RPM at approximately 3 load cycles per minute as represented in Fig. 75. The spin pit designed by Test Devices Inc. features a lead brick liner surrounded with high strength steel casing for containment, and also includes a vacuum system which provides a vacuum level below 60 Pascal maintained through airtight seals. The fan dovetail assembly is powered through a Barbour Stockwell Inc. '8-inch' turbine. The low frequency loading (Fig. 75) is attributed to mechanical as well as electrical power limitations required to accelerate and decelerate the large mass moment of inertia of the fan assembly. Based on a previous investigation of frequency effects in fretting wear [74], wear damage was shown to be independent of frequency for low amplitude fretting due to low interfacial strain rates. Since the tests were conducted in controlled room temperature environments at low frequency, it may be concluded that the fretting behavior of the dovetail fan was isolated from any possible temperature and frequency effects. Furthermore, the tests were conducted in a vacuum environment, eliminating the opportunity of the fan blade being subjected to HCF type loading from aerodynamic excitation. Instead, the fretting motion between the dovetail fan blade and hub was a result of varying centrifugal loading for the defined speed range.

The dovetail fan blade and hub in question are made of titanium alloy, Ti-6Al-4V, with an approximate root chord length of $125 \mathrm{~mm}$ and blade mass of $1 \mathrm{~kg}$. The fan was disassembled at each defined inspection interval followed by eddy current and Fluorescent Penetrant Inspection (FPI) at critical high stress and fretting susceptible locations on the fan hub Fig. 74 (b) and fan blade Fig. 74 (c). Following inspection, the dovetail fan was reassembled and testing was resumed until crack detection. From the three separate tests, a total of four independent cracks Fig. 54, cracks A$\mathrm{D}$, were detected on the hub portion of the fan dovetail attachment, concentrated mostly at the LE pressure side slot, roughly $7 \mathrm{~mm}$ away from the LE end face. The unopened cracks Fig. 54 (a) showed the crack propagation path to be approximately normal to the contact surface. The cracks were opened Fig. 54 (b) to observe the fracture surface characteristics. The surfaces produced similar and well defined arc shapes with dark colored fracture surfaces and an aspect ratio less than one. The surfaces exhibited ridges and beach marks, characteristic of fatigue initiation and propagation. Crack A displayed multiple origin sites along the slot which propagated into the dovetail. A similar investigation conducted by D. Garcia et al. [60] on Ti-6Al-4V showed multiple fretting cracks initiating along the contact surface. However, it was concluded that the life of the component was governed from a single dominant crack [60]. No original metallurgical abnormalities were found at the origin sites and the material of the part was consistent with compositional requirements, thus concluding that material anomaly was not the root cause of the fractures. Severe 
fretting Fig. 77 was evident along all of the slot surfaces at both the leading and trailing edges. Fourier Transform Infrared Spectroscopy (FTIR) and EDS identified a black deposit composed of a base metal oxide at the contaminated dark colored fracture surface region. Similar fractographic investigation done by S. Barella et al. on a third stage turbine blade failure [22] revealed brownish marks detected at the fatigue origin site, which were suggested to be characteristic of fretting fatigue damage as a result of accumulation of wear debris from repetitive contact between the blade and steel rotor disk. Striation counts were performed on the opened fracture surfaces using EDS and micrometer readings and are shown in Fig. 78. For some of the fracture surfaces, due to contamination resulting from fretting close to the origins, the striations could not be accurately resolved. For these cases, only the striations which could be accurately measured were reported. Overall, the scatter of the striation counts show the crack growth rates linearly increasing with crack depth on a logarithmic scale, characteristic of LEFM.

To distinguish between the crack nucleation stage and propagation phase, an initial crack size of $760 \times 380 \mu \mathrm{m}$ was considered to characterize the crack nucleation. This size of crack is based on typical eddy current detection limits, and is consistent with the initial size assumed by Lykins et al. [24]. In this current study, the propagation phase of the fretting crack is determined through fatigue striations exhibited on the fracture surface. The nucleation stage is determined by subtracting the propagation cycles from the number of cycles endured in the spin test upon scheduled inspection.

(a)

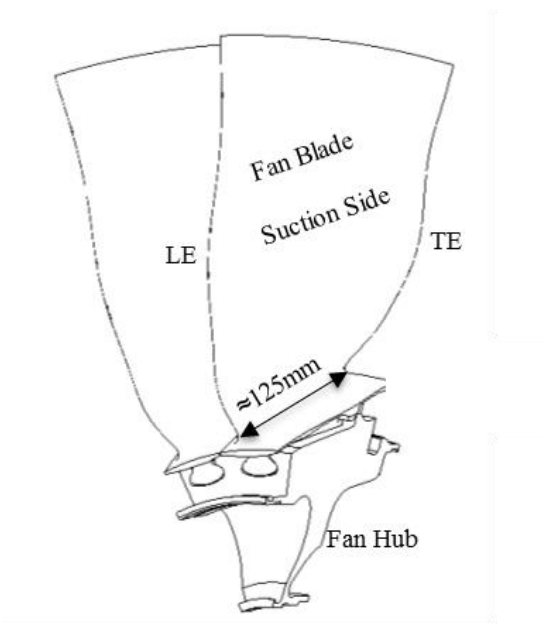

(b)

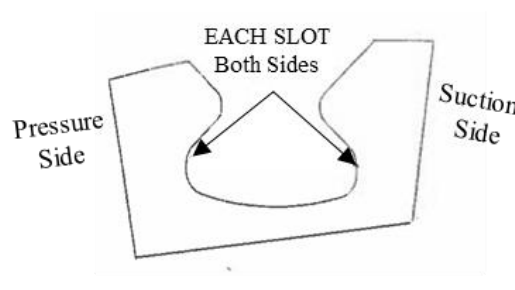

(c)

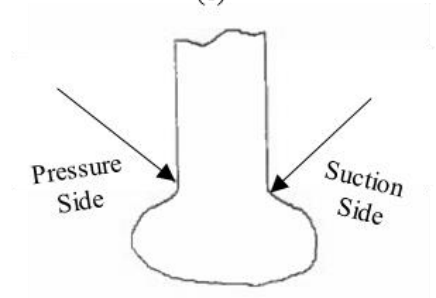

Fig. 74. (a) Two-blade sector (b) inspection locations on hub (c) inspection locations of the blade 


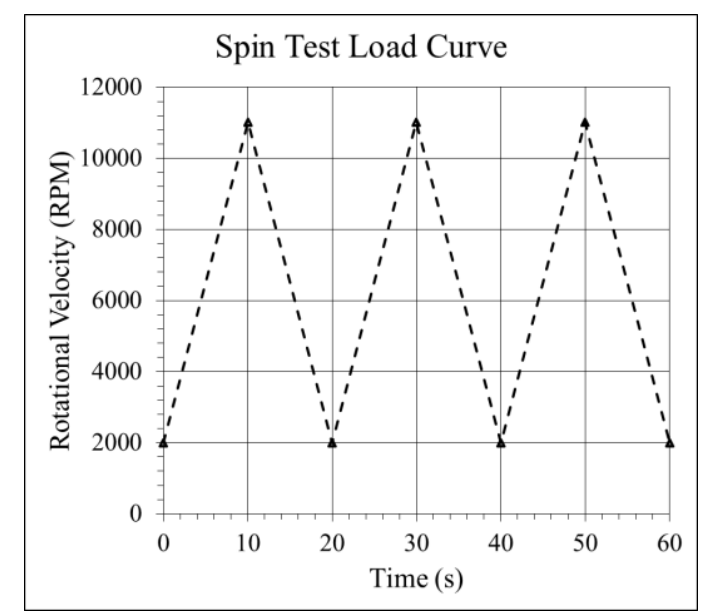

Fig. 75. Representative load curve used for the spinning tests

(a)
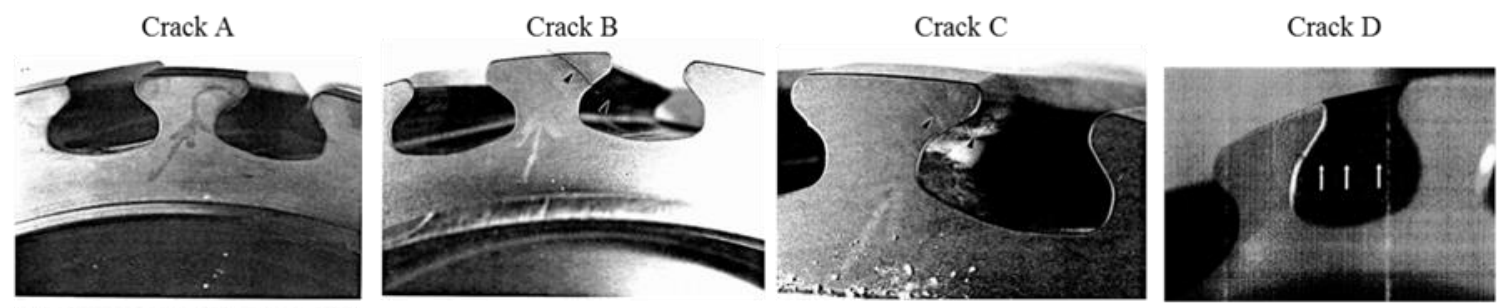

(b)
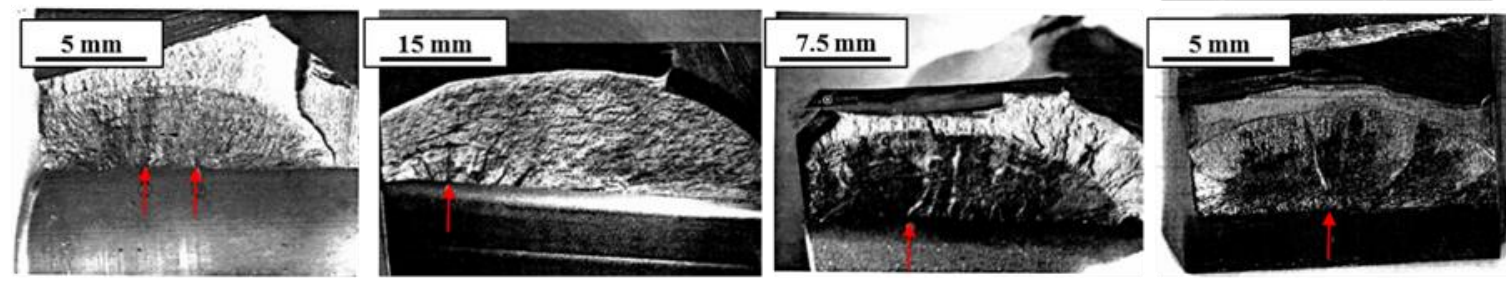

Fig. 76. Four cracks detected from three individual spin tests concentrated at LE pressure side slot

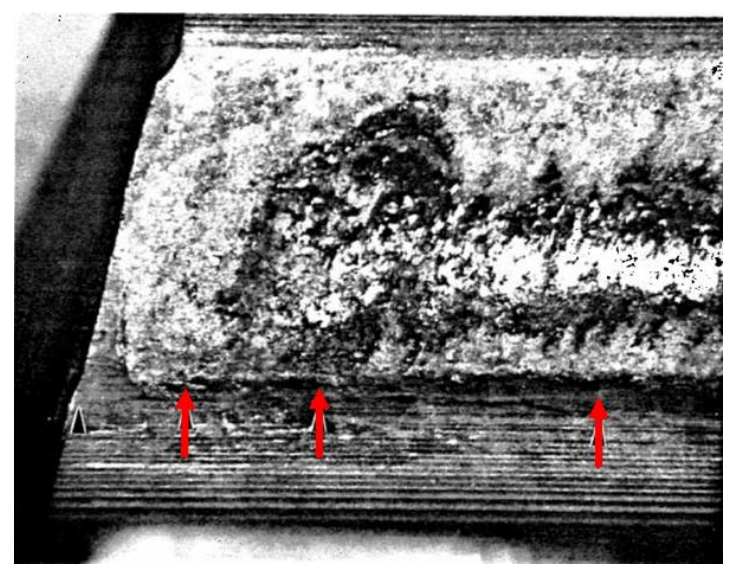

Fig. 77. Severe fretting along slot surface (Crack A) present on all slots at both the leading and trailing edges 


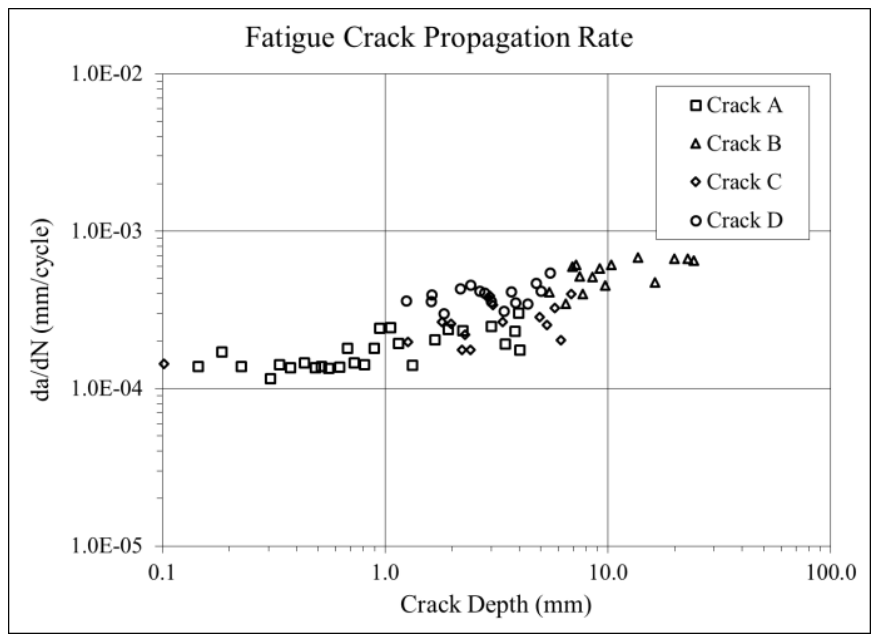

Fig. 78. Striation counts on the opened fracture surfaces for Cracks A-D using EDS and micrometer readings

\subsection{FINITE ELEMENT MODELING AND SUBMODELING}

Factors present in fretting including wear, adhesion, asperity deformation, and the evolution of COF due to an increasing number of cycles are usually ignored when modeling fretting fatigue [24], [59]. These factors can result in a COF which is much higher than initially measured or predicted. Thus, using larger values for the COF has often resulted in better calibration of analytical with experimental results [59]. Based on work done by PJ. Golden [75], the $\mathrm{COF}$ value for Ti-6Al-4V alloy tested at room temperature varied from 0.3 to 0.7 [75]. In this study, a similar range of COF is prescribed in the FE model between the fan blade and hub to study the sensitivity of stresses as well as the overall crack growth behavior. Since fretting is a HCF related issue with the crack growing in elastic stress fields, elastic material properties have been widely used in the past to model the stresses and crack growth near the contact area [8], [18] and thus, was also considered for this work. At room temperature, the approximate tensile properties for a representative Ti-6Al-4V alloy are $v=0.33, \mathrm{E}=114 \mathrm{GPa}$, and $\sigma_{\mathrm{y}}=1100 \mathrm{MPa}$.

A 3D ANSYS FE model with a fine hexahedral SOLID186 mesh was constructed using linear elastic material properties at room temperature, with two unique COF values $(0.35,0.70)$ between the fan blade and hub, and with large displacement turned on. The stress converged model Fig. 79 (a) had a total of 1.3 million nodes, requiring more than 24 hours to solve for two load steps on 8 CPUs. The two load steps used in the analysis correspond to the tested speed range of 2000 RPM and 11000 RPM. A two-bladed sector was used for the analysis by taking advantage of the periodic boundary conditions at the cut surfaces which take into account the symmetry of the fan hub. Contact was established through ANSYS using CONTA174 and TARGE170 elements, utilizing the Asymmetric AugmentedLagrange contact formulation. The max principal stresses on the fan hub for the max speed condition is shown for a COF of 0.35 and 0.70 in Fig. 79 (b) and (c) respectively. The stresses at the dovetail contact area are a result of the centrifugal forces in the hub due to its inertia during operation and the centrifugal force generated by the $1 \mathrm{~kg}$ mass of the blade, which is reacted at the suction and pressure side of the dovetail fan hub through a combination of friction and contact pressure. When the COF decreases, the contact pressure increases to maintain equilibrium, resulting in larger elastic deformations and slip amplitudes. Conversely, when the COF increases, the contact pressure decreases and the shear tractions increase to maintain the equilibrium state. At 11000 RPM, the centrifugal force generated by 
the blade is equivalent to 290000 Newtons. Under this loading, the FE results indicate a peak steady stress near the LE pressure side of the hub, at the trailing (bottom) edge of contact. A path was created in ANSYS Fig. 80 (a), starting from the peak steady stress location determined from FE, following the crack propagation path observed from tests in Fig. 54 (a). The max principal stresses (S1) were extracted along the path, and are shown in Fig. 80 (b). The results show that between depths of $0 \mathrm{~mm}$ to $3 \mathrm{~mm}$, the principal stresses in the bulk of the fan hub are sensitive to the contact conditions imposed by the COF. After $3 \mathrm{~mm}$, the effect of the COF on the principal stresses in the bulk of the fan hub become negligible. This closely reflects observations documented in [14], which suggests that oblique cracks at the early stages of crack growth can range from tens of microns up to $3 \mathrm{~mm}$. The dominant normal stresses in the radial (Sx) and hoop (Sy) directions defined by the cylindrical coordinate system, as well as the dominant shear stresses in the XY plane were also extracted along the path, and are shown in Fig. 80 (c), (d), and (e) respectively. These results show that a larger COF value increases the dominant stresses near the edge of contact but its effect on stresses diminishes along the bulk of the fan hub.

Since FRANC3D is a discrete crack growth analysis tool which requires remeshing and solving for each crack iteration, a coarsened submodel was essential to speed up the analysis time. A submodel was constructed shown in Fig. 81 (a) consisting of the fan hub, a single blade with $90 \%$ of the airfoil removed, and imposed displacements from the full model solution at the cut boundaries. The contacts in the submodel were defined identically to the full model. A check was done shown in Fig. 81 (b) to ensure that the stresses and contact conditions captured by the coarsened submodel were numerically identical to the full model solution. Using the same path defined in Fig. 80 (a) and extracting the max principal stress, it was concluded that the submodel accurately captured the stresses in the bulk of the material. A study was also done to ensure that the total load captured by the submodel was equal to the full model even as the crack grew larger in size. This was originally a concern since the submodel uses constant imposed displacements for the pristine crack-free solution. Thus, it was thought that as the crack grows the structure becomes less stiff and as a result less load is captured in the model. However, even for the largest sized crack, the submodel retained $95 \%$ of the original load, and thus, the submodel approach was considered an adequate approximation. The submodel required only 20 minutes to solve for each crack iteration compared to the 24 hours required by the full model.

(a)

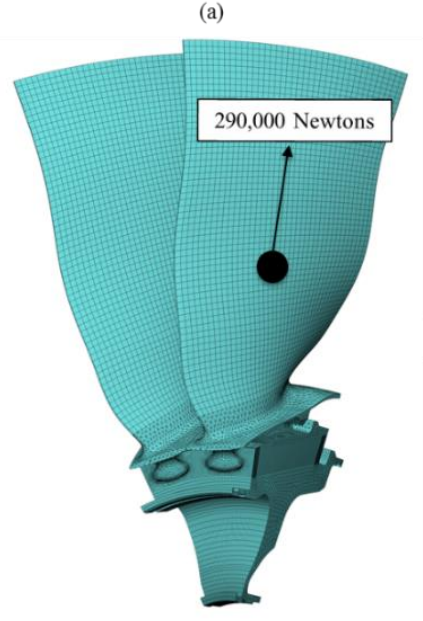

(b)

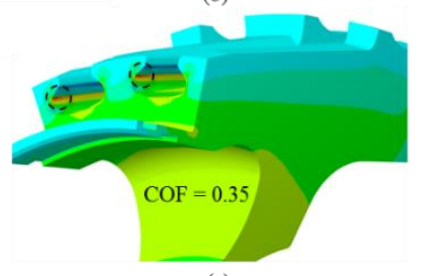

(c)

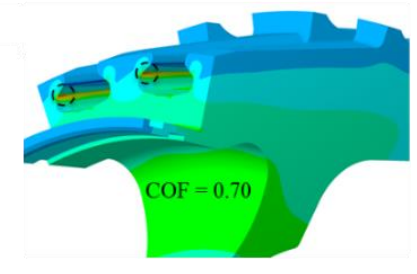

Fig. 79. (a) Two-bladed sector FE model. Max principal stress plot for hub for (b) $\mathrm{COF}=0.35$, (c) $\mathrm{COF}=0.70$ 
(a)
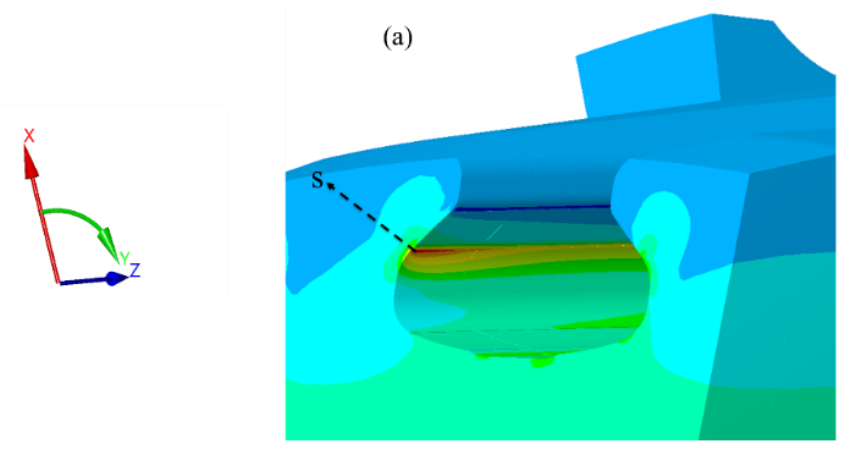

(b)

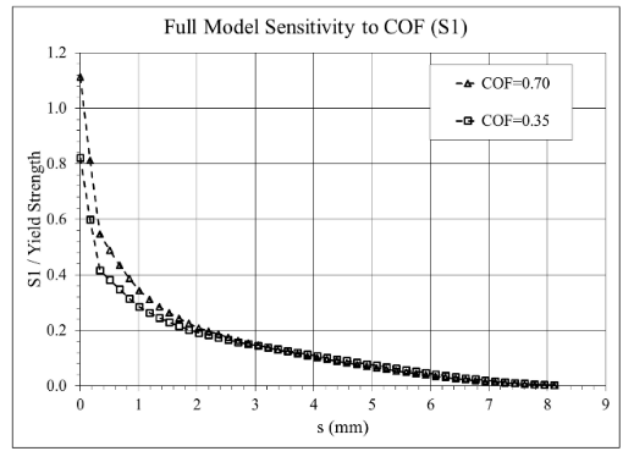

(d)

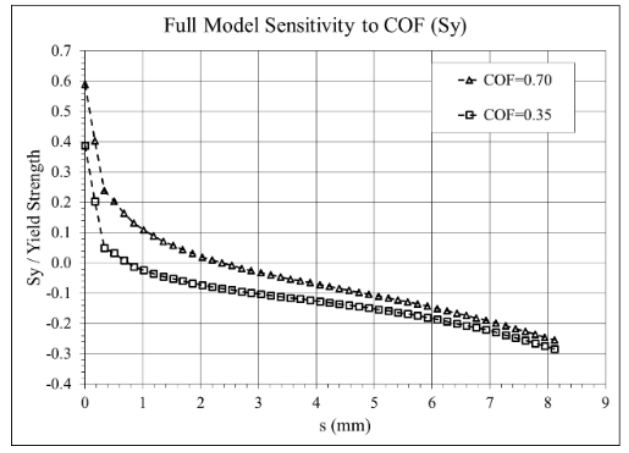

(c)

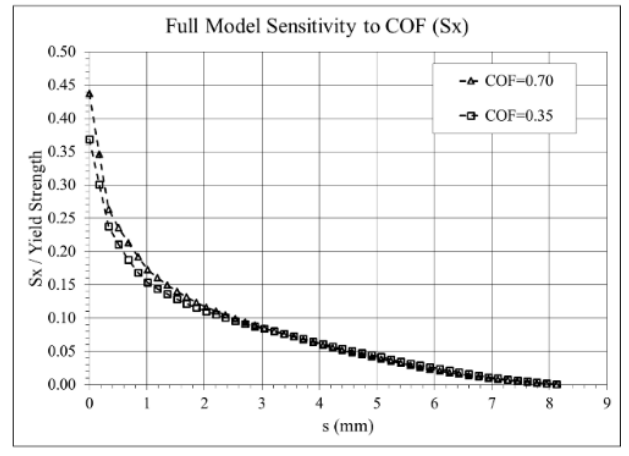

(e)

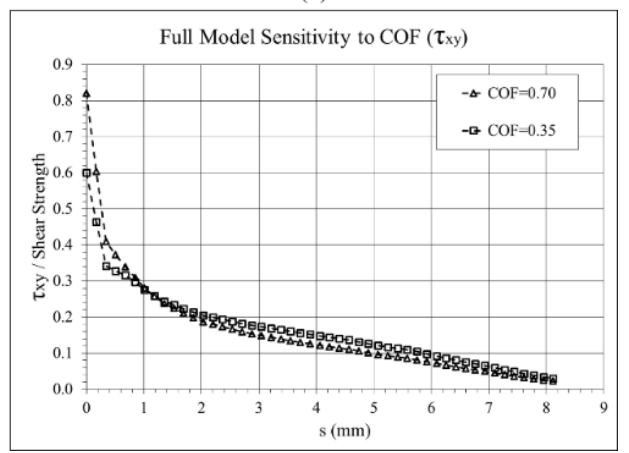

Fig. 80. (a) Path (b) to (e) Principal, radial, hoop and shear stresses along path for COF of 0.70 and 0.35

(a)

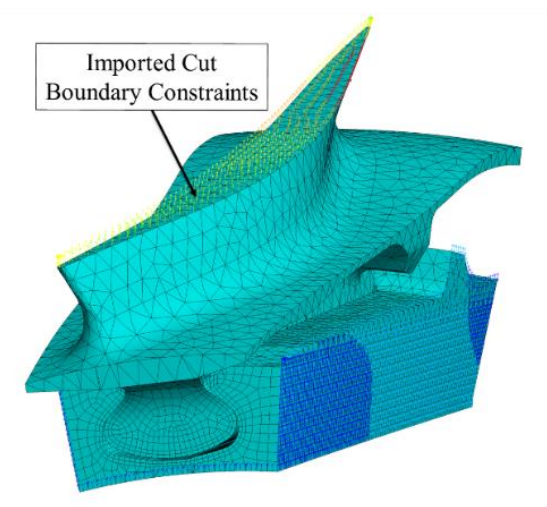

(b)

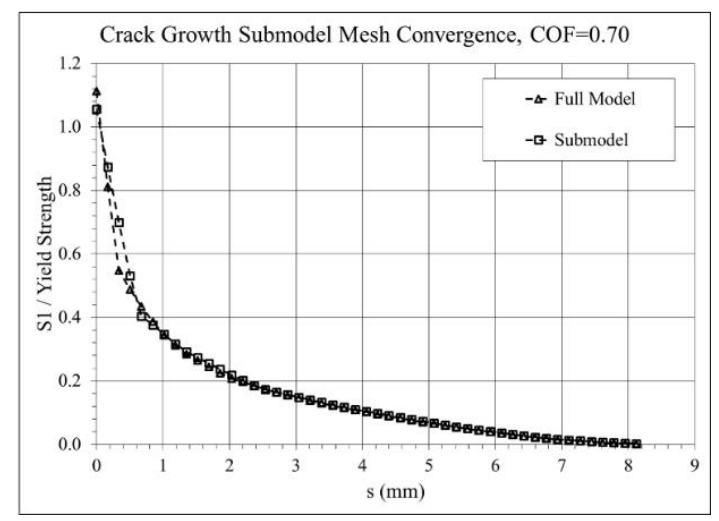

Fig. 81. (a) Coarsened submodel consisting of the hub, blade (b) Stresses along crack propagation path 


\subsection{CRACK GROWTH SIMULATION}

Modeling the fretting fatigue behavior for the dovetail fan attachment required a representative value for an evolved COF between the fan blade and hub at a time corresponding to crack nucleation. This was done through calibration of 3D crack growth simulations for various $\mathrm{COF}$ with respect to the fractured components in regards to the simulated crack trajectory, aspect ratio, shape, and propagation life. It was theorized that since the principal stresses along the crack propagation path from $0 \mathrm{~mm}$ to $3 \mathrm{~mm}$ are dependent on the prescribed COF value, as shown in Fig. 80 (b), then the crack growth behavior also should be dependent on the COF. As such, the resulting crack shape, aspect ratio, and propagation rates observed from tests have embedded within them the physical consequences of the evolution of the COF from the start of the test up until the crack reached a depth of $3 \mathrm{~mm}$.

As mentioned earlier, the crack growth simulations were modeled for a COF value of 0.35 and 0.70 through FRANC3D using the initial submodel constructed in Fig. 81 (a). A single initial semi-circular shaped crack with a radius of $125 \mu \mathrm{m}$ and an aspect ratio of one was inserted near the LE pressure side at the bottom edge of contact, 7 $\mathrm{mm}$ away from the LE end face in the chord wise direction as shown in Fig. 82. This corresponds to the crack nucleation locations observed from the four independent fractures in Fig. 54 (b). The initial flaw size chosen for the simulation is larger than the critical flaw size of $50 \mu \mathrm{m}$ determined for titanium alloys [68], thus, long crack da/dn vs $\Delta \mathrm{K}$ data was considered sufficient for characterizing the crack growth behavior. For this study, the influence of the initial crack size on the overall crack propagation life was not studied. Based on previous studies [63], [76], it has been concluded that the fretting fatigue crack growth behavior of Ti-6Al-4V specimens is insensitive to the size of the initial crack due to very high initial crack growth rates. One study in particular found that for initial crack sizes larger than $50 \mu \mathrm{m}$, the propagation life is independent to the size of the initial crack [65]. It is also worth noting that although Crack A exhibited multiple origin sites on the fracture surface, the simulation only considered a single dominant crack. This simplification is considered appropriate based on findings from previous investigations of fretting fatigue cracks for Ti-6Al-4V specimens [60], which showed a single dominant crack to be the determining factor to the fracture for the majority of the components, even when multiple fretting cracks were identified along the contact surface.

For both of the crack growth simulations (COF values 0.35 and 0.70), the MTS method was used to predict the crack trajectory. The MTS method was chosen as it has been used successfully in the past for simulating crack growth of a similar Ti-6Al-4V dovetail fan attachment [8]. The crack was grown up to the limits of the FE model with respect to the geometry and accuracy of the submodel approach as the crack grew larger in size. A total of 40 discrete crack iterations were performed to steadily grow the crack from the initial $125 \mu \mathrm{m}$ depth up to a final depth of approximately $6 \mathrm{~mm}$. The visual representations of the crack front advancements produced by the simulation, including the side view of the final cracks are shown in Fig. $\mathbf{8 3}$ for both modeled COF values. The opened fracture surface for Crack A was compared to the crack mouth from the simulation for the corresponding crack size. The dashed lines represent a trace of the opened crack front of the fractured fan hub. Superimposing the trace on the predictions show that FRANC3D accurately captured the aspect ratio and elliptical shape of the crack for both of the studied COF values. Similarly, when comparing the side view of the simulated cracks with the unopened view of Crack B, it was concluded that FRANC3D accurately captured the crack growth trajectory using the MTS method. However, using a COF value of 
0.70 resulted in a crack trajectory and aspect ratio which aligned slightly closer to the fractured components compared to a COF value of 0.35 .

From the crack growth simulation, M-Integral stress intensities calculated by FRANC3D along the crack depth shown in Fig. 84 were used to predict crack growth lives by utilizing the FNK model [47]. The FNK model, represented by equation (106) accounts for the retardation near threshold, acceleration near fast fracture and crack closure effects. The $\mathrm{C}, \mathrm{n}, \mathrm{p}$, and, $\mathrm{q}$ are empirical constants derived by curve fitting test data at varying environmental conditions such as temperature and varying load ratios. $\Delta \mathrm{K}$ is the applied stress intensity factor range along a path of all crack front advancements, $\Delta K_{t h}$ is the threshold stress intensity range which is dependent on loading ratio, $\mathrm{K}_{\mathrm{c}}$ is the critical stress intensity factor, and $\mathrm{R}$ and $\mathrm{f}$ represent the load ratio and crack closure function respectively. The fatigue crack growth properties for a representative Ti-6Al-4V alloy taken from the FRANC3D material database are $\mathrm{C}=4.466 e^{-13}(\mathrm{da} / \mathrm{dN}$ in $\mathrm{mm} / \mathrm{cycle}, \Delta \mathrm{K}$ in $\mathrm{MPa} \sqrt{\mathrm{mm}}), \mathrm{n}=3.2, \mathrm{p}=0.25$, and $\mathrm{q}=0.75$. It is noted that the $\mathrm{K}_{\min }$ corresponding to $\Delta \mathrm{K}$ used for the computation of the crack propagation rates was based on a fully unloaded condition, rather than the actual 2000 RPM load condition during testing. This was done to speed up the analysis time in FRANC3D by limiting the number of load steps that required solving. This simplification was considered acceptable given that the load ratio between the min and max load step was very low $(\mathrm{R}=0.03)$, and since the proportionality of the stresses was not greatly affected between the two operational speeds.

$$
\frac{d a}{d N}=\frac{C(1-f)^{n} \Delta K^{n}\left(1-\frac{\Delta K_{t h}}{\Delta K}\right)^{p}}{(1-R)^{n}\left(1-\frac{\Delta K}{(1-R) K_{c}}\right)^{q}}
$$

Fig. 85 shows the predicted crack growth rates for both of the studied COF values along the crack depth compared to collected test data. It was noticed that for both $\mathrm{COF}$, the predicted crack growth rates at the very early stages of crack growth did not agree with the measured striations. However, after reaching a crack depth of $400 \mu \mathrm{m}$, the COF of 0.70 simulation resulted in crack growth rates which agreed very well with the test data. The crack propagation life for the simulations and experiment, shown in Fig. 86, were calculated through numerical integration of the crack growth rates with the crack depth. The resulting crack propagation lives show that the COF value of 0.70 results in more accurate predictions of the crack propagation life compared to a COF of 0.35 . The final crack modeled in both simulations had a crack depth of approximately $6 \mathrm{~mm}$. At this corresponding crack size, the crack propagation life when using a COF of 0.70 was predicted to be $55 \%$ of the total propagation life determined from tests. This agrees well with the test data which shows the propagation life to be $46 \%$ of the total propagation life for the same crack size of interest. Based on the performed crack growth simulations, it is concluded that prescribing a COF value of 0.70 results in better predictions of the crack trajectory, aspect ratio, shape, growth rates, and propagation life. Furthermore, given that the value was obtained through calibration of the crack growth simulations, the test results have embedded in them the physical consequences of the evolved COF that results from an increasing number of fretting cycles at the time of crack nucleation. This COF value will therefore be employed for the prediction of the fretting fatigue behavior of the dovetail fan assembly. 


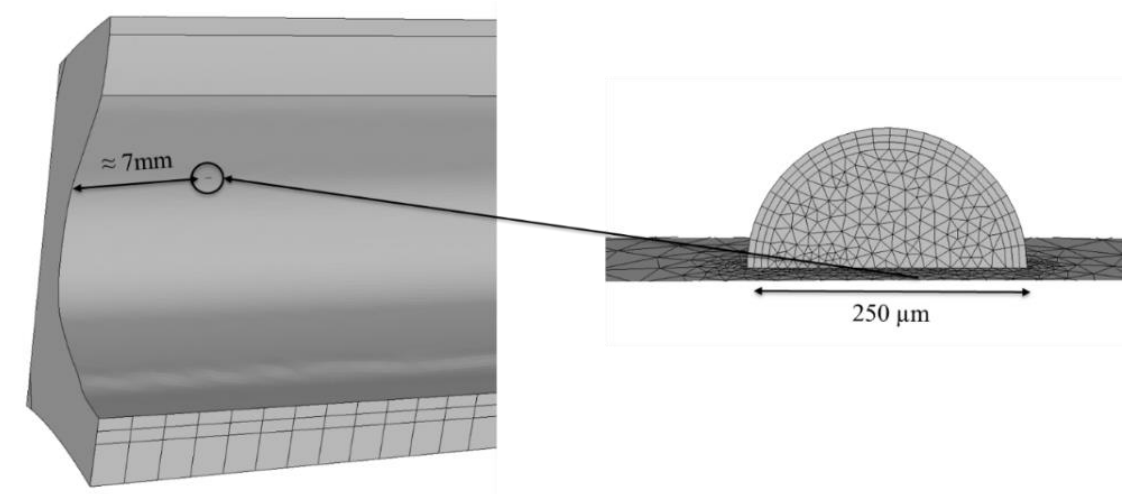

Fig. 82. Initial semi-circular shaped crack with radius $125 \mu \mathrm{m}$ inserted at the LE pressure side

Crack A

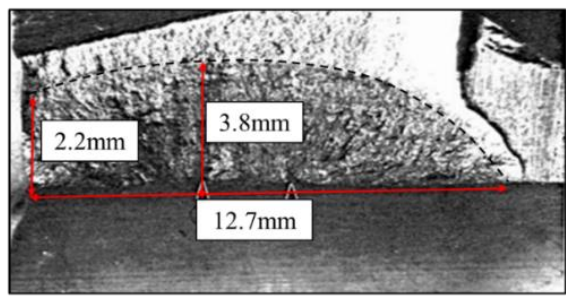

$\mathrm{COF}=0.35$

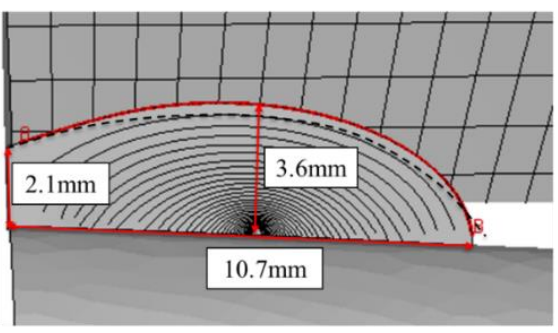

$\mathrm{COF}=0.70$

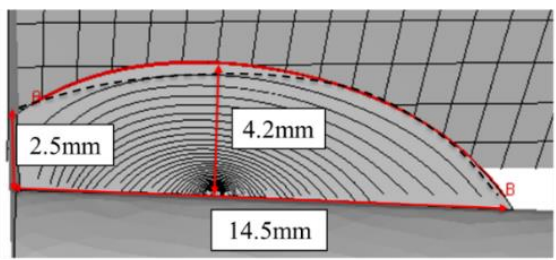

Crack B

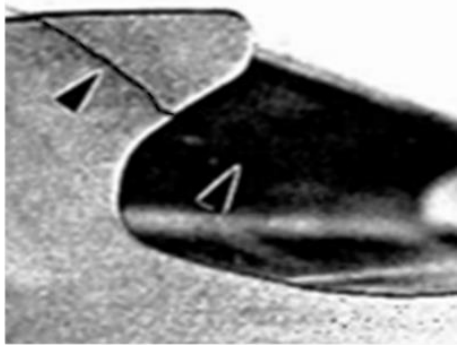

$\mathrm{COF}=0.35$

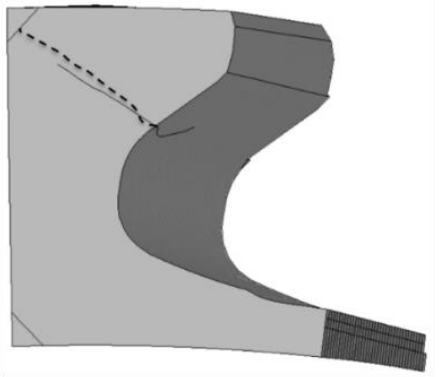

$\mathrm{COF}=0.70$

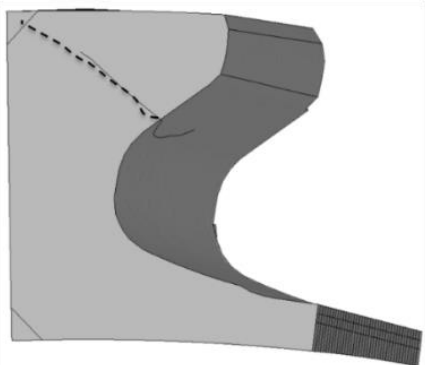

Fig. 83. Outputs of FRANC3D crack growth simulations 

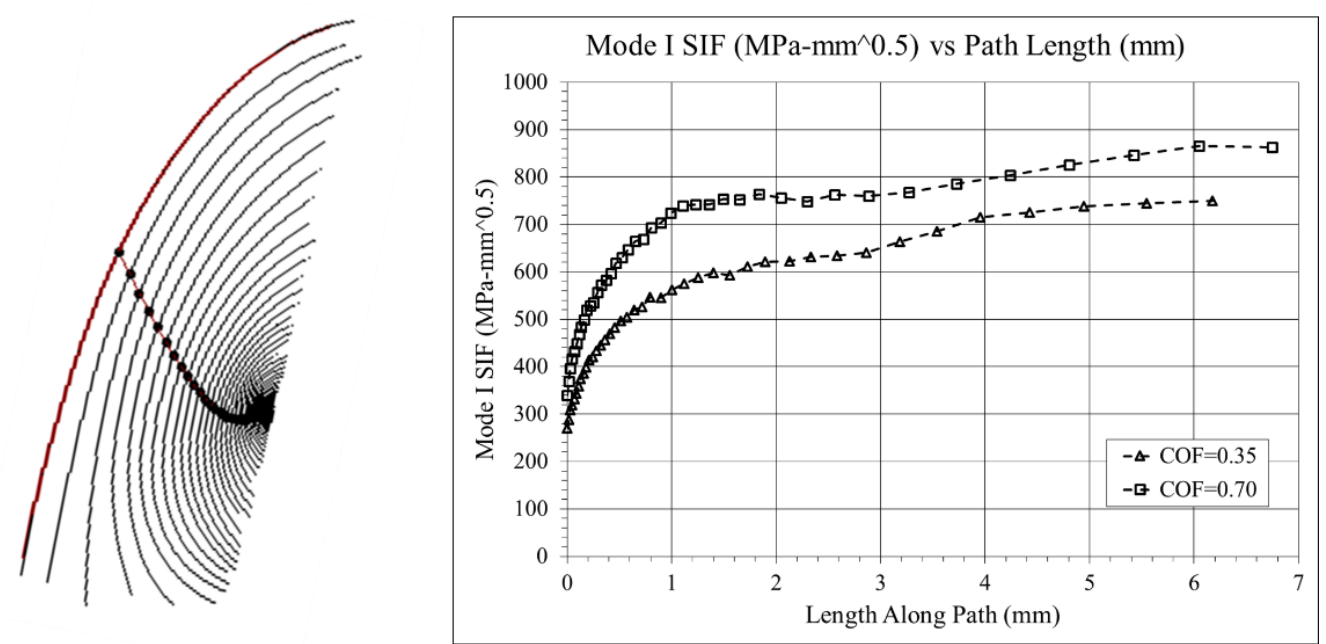

Fig. 84. Mode I SIF along a defined crack propagation path for both COF of 0.35 and 0.70

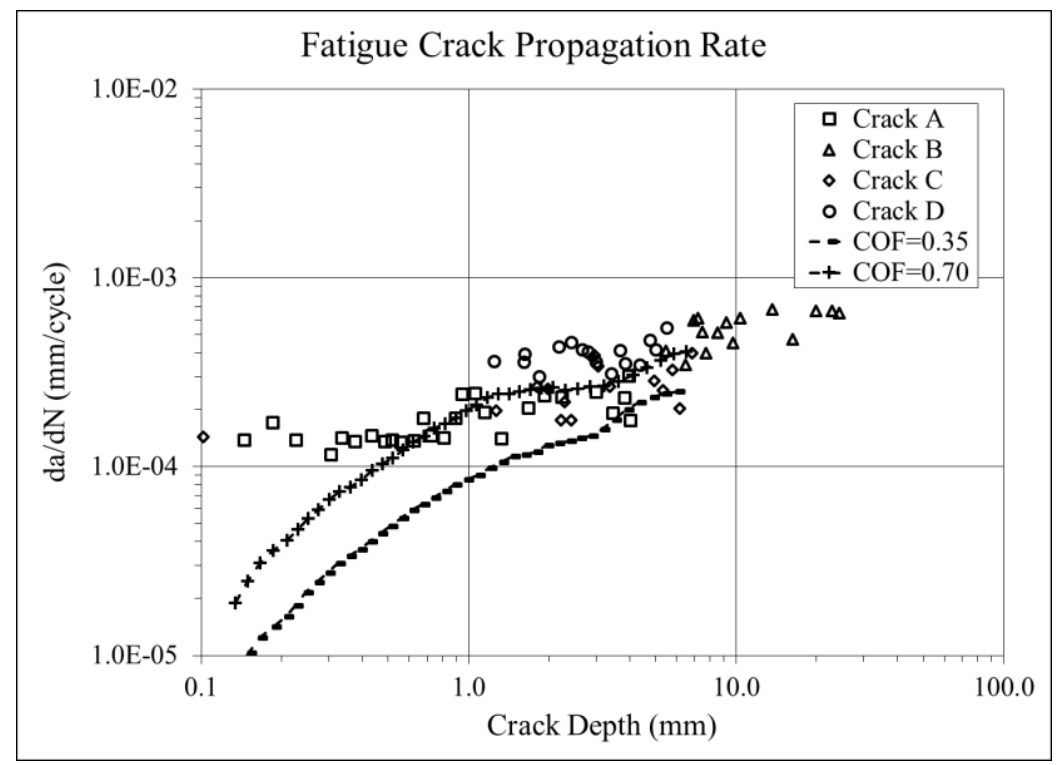

Fig. 85. Predicted crack growth rates compared to test measurements 


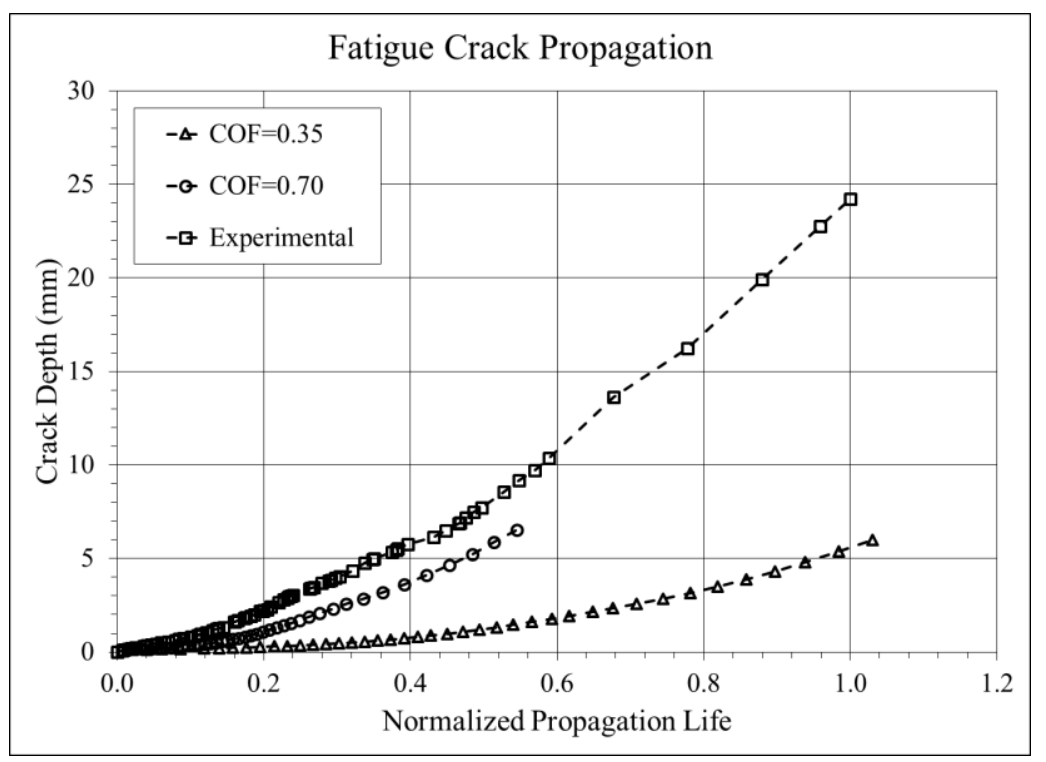

Fig. 86. Crack propagation lives of simulations compared with experimental

\subsection{FRETTING FATIGUE}

The fretting fatigue behavior of the dovetail fan attachment was studied using two fretting nucleation models. The first model is the SWT parameter represented by equation (108). The SWT parameter is a critical plane approach considered for cracks nucleating at a plane where the product of the normal strain range $\Delta \varepsilon$ and maximum normal stress $\sigma_{\max }$ is a maximum. The $E$ is the elastic modulus, $\sigma_{f}^{\prime}$ and $\varepsilon_{f}^{\prime}$ are material strength parameters, and $b$ and $c$ are curve fitting parameters. For Ti-6Al-4V, the corresponding properties taken from [18], [77] are $\sigma_{f}^{\prime}=376.6 M P a$, $\varepsilon_{f}^{\prime}=34.4, b=1.78 E-4$, and $c=-0.767$. FRANC3D calculates the SWT parameter for all planar orientations using 2D stress transformation equations (107), and ignores out of plane components by rotating the 3D stresses and strains from the FE model into a unique coordinate system defined for each node in contact. The $x$ and $y$ directions of the rotated coordinate system correspond to the computed sliding direction vector $s$ and the normal $n$ to the contact element. This is done to replicate a conventional fretting test, in which the direction of the sliding vector and applied tensile loading are parallel. The second studied parameter is the modified SWT parameter [33], developed for fretting fatigue specific applications. The modified SWT parameter, represented by equations (109) and (110), assumes that damage resulting from fretting fatigue is the combination of damage associated to frictional wear $D_{\text {fret }}$ which results in the nucleation of a small crack below the contact surface, and the SWT parameter which drives the growth of the crack. The magnitude of frictional work $\tau \delta$ imbedded in the $D_{\text {fret }}$ parameter accelerates crack nucleation. As such, the predicted fretting fatigue life is reduced even for a small SWT parameter if the wear parameter is large. The model incorporates a threshold limit $(\tau \delta)_{t h}$ to distinguish whether the failure is governed by fretting fatigue or fretting wear. As frictional work approaches the threshold, fretting wear becomes more probable than fretting fatigue and embryo cracks are worn away before having the opportunity to grow to failure. The $\mathrm{m}, \mathrm{n}$, and $(\tau \delta)_{\text {th }}$ on the right hand side of equation (110) are material constants determined through experimentation. The right hand side of equation (109) is identical to the plain fatigue SWT parameter. For Super CMV material, a representative value for $(\tau \delta)_{\mathrm{th}}$ is $1.36 \mathrm{MPa}$ - 
$\mathrm{mm}$, and $\mathrm{m}$ and $\mathrm{n}$ obtained through trial and error procedure were found to be 0.25 and 3.7 [33]. For this work, in the absence of more appropriate experimental values, the $(\tau \delta)_{\text {th }}$ for the Ti-6Al-4V alloy was kept equal to that of Super CMV material. This assumption, although less than optimal, provided a reasonable starting point to compute the modified SWT parameter. For conservatism, the remaining $\mathrm{m}, \mathrm{n}$ material constants were obtained using a trial and error procedure on the lowest obtained experimental fretting nucleation life. Since the tests presented only a single calibration criterion, the obtained $\mathrm{D}_{\text {fret }}$ parameters naturally produced a fretting fatigue life in very close agreement with test results. Therefore, the aim of this study was to merely show the capability of the modified SWT parameter in accurately predicting the fretting fatigue behavior for a single and unique geometry and load case. Further testing is required to validate the obtained $\mathrm{D}_{\text {fret }}$ parameters on the Ti-6Al-4V alloy for other loading conditions and unique dovetail fan attachments. This however was considered to be outside the scope from the current body of work.

$$
\begin{gathered}
\sigma=\frac{\sigma_{\mathrm{xx}}+\sigma_{\mathrm{yy}}}{2}+\frac{\sigma_{\mathrm{xx}}-\sigma_{\mathrm{yy}}}{2} \cos 2 \theta+\tau_{\mathrm{xy}} \sin 2 \theta \\
\frac{\Delta \varepsilon}{2} \sigma_{\max }=\frac{\left(\sigma_{\mathrm{f}}^{\prime}\right)^{2}}{\mathrm{E}}\left(2 \mathrm{~N}_{\mathrm{f}}\right)^{2 \mathrm{~b}}+\sigma_{\mathrm{f}}^{\prime} \varepsilon_{\mathrm{f}}^{\prime}\left(2 \mathrm{~N}_{\mathrm{f}}\right)^{\mathrm{b}+\mathrm{c}} \\
\sigma_{\text {max }} \frac{\Delta \varepsilon}{2} D_{\text {fret }}=\frac{\left(\sigma_{f}^{\prime}\right)^{2}}{E}\left(2 N_{f}\right)^{2 b}+\sigma_{f}^{\prime} \varepsilon_{f}^{\prime}\left(2 N_{f}\right)^{b+c} \text { for } \tau \delta \leq(\tau \delta)_{\text {th }} \\
D_{\text {fret }}=(1+\tau \delta)^{m}\left\langle 1-\frac{\tau \delta}{(\tau \delta)_{t h}}\right\rangle^{n}
\end{gathered}
$$

To accurately model the fretting fatigue of the dovetail fan attachment, a very fine mesh was used for the FE model near the contact area to ensure converged tangential displacements $\delta$ at the edges of contact. This is in contrast to the mesh used for the crack growth analysis, which was mainly dependent on converged stresses in the bulk of the fan hub. To this effect, a fretting submodel was constructed for the hub with the entire fan blade modeled consisting of a total of 1.7 million nodes with an element size of $70 \mu \mathrm{m}$ at the edges of contact and a prescribed COF value of 0.70 obtained through crack propagation analysis. Since fretting is a non-linear and history dependent phenomenon due to friction, multiple loading cycles have been previously used for stabilization of the partial slip cycles [26], [58], [59]. In an effort to generate a closed friction hysteresis response, the submodel was cycled from 2000 to 11000 RPM for a total of six cycles. The final model solved in 12 hours when running on 48 CPUs. The tangential displacements shown in Fig. 87 (a) were probed near the edge of contact, close to the crack nucleation locations observed from tests. The probed response showed that when angular velocity was increased from 0 to 2000 RPM, the disk slot only slightly opened, and the blade slipped a negligible amount. When the angular velocity was further increased from 2000 RPM to 11000 RPM, the dovetail slot further opened up resulting in an increase in slip, with tangential amplitudes approaching gross slip conditions. Decelerating the speed to 2000 RPM resulted in a different locking position of the blade and thus, contact conditions which were unique to the initial 2000 RPM ramp up. As such, when the load was reversed, the tangential amplitudes were more representative of partial slip conditions, and the frictional response displayed narrower hysteresis loops. The slip magnitudes shown in Fig. 87 (b) were calculated for each load step by subtracting the accumulated sliding distance. The first speed cycle shows a tangential displacement of approximately $85 \mu \mathrm{m}$, with the remaining speed cycles ranging from 55 to $65 \mu \mathrm{m}$. Since the refined 3D model required expensive computational time and resources, six loading cycles were considered sufficient for obtaining stabilized sliding 
amplitudes at the edges of contact near the observed crack nucleation location. The FE solution containing the stresses, strains, and contact solutions were passed along to FRANC3D for the fretting analysis. The fretting nucleation parameters were calculated using the last two load steps corresponding to the final speed cycle.

The plain fatigue SWT parameter was calculated by FRANC3D at both the pressure side and suction side of the fan hub, shown in Fig. 88 (a) and (b) respectively. Based on the SWT parameter, the critical fretting nucleation location was predicted to be on the suction side, roughly mid chord along the dovetail contact face. This does not agree well with test results, in which the four fractures were concentrated at the pressure side and close to the LE. The fretting specific modified SWT nucleation model was calculated by FRANC3D by taking $(\tau \delta)_{\text {th }}=1.36 \mathrm{MPa} \mathrm{mm}$ to be the frictional work threshold, and $\mathrm{m}=1$ and $\mathrm{n}=0.4$ for the material fitting parameters. These $\mathrm{m}$ and $\mathrm{n}$ parameters were obtained through a trial and error procedure by varying the parameters until the predicted crack nucleation location and fretting nucleation life agreed well with test results. Fig. 89 shows the resulting modified SWT nucleation parameter calculated at the pressure and suction side of the fan hub. The critical location was predicted to be on the pressure side, and a region of high magnitude was present at the LE pressure side, centralized between $2 \mathrm{~mm}$ and 8 $\mathrm{mm}$ from the end face in the chord wise direction. This agreed more closely with the fractured components compared to predictions made by the plain fatigue SWT parameter. In further comparing Fig. 88 (a) and Fig. 89 (a), it is also observed that the band of material susceptible to fretting fatigue is reduced under the modified SWT parameter. This location 'filtering' effect is a direct outcome of the $D_{\text {fret }}$ parameter. When the frictional work approaches the frictional threshold limit $(\tau \delta) /(\tau \delta)_{t h} \rightarrow 1$, the $D_{\text {fret }}$ parameter decreases significantly and approaches zero, implying that failure for these locations is governed by fretting wear rather than fretting fatigue. The influence of the COF to the predicted crack nucleation location can be understood through the same logic. For instance, prescribing a larger COF value would increase the $(\tau \delta) /(\tau \delta)_{t h}$ parameter and further reduce the band of material susceptible to fretting fatigue. This perhaps would 'filter' out locations previously predicted to be critical and lead to predictions of new nucleation sites

The nucleation lives were calculated at the critical LE pressure side location using both nucleation models shown in Fig. 90 (a). The plain fatigue SWT parameter predicted the life to be an order of magnitude larger than the lowest experimental nucleation life. Since the $\mathrm{D}_{\text {fret }}$ parameters were adjusted through a trial and error procedure considering a single calibration point, the predicted and lowest experimental nucleation life (Crack B) were in good agreement based on the modified SWT parameter. Fig. 90 (b) shows the resulting $D_{\text {fret }}$ curve, and the magnitude of $D_{\text {fret }}$ at the corresponding LE pressure side location. 
(a)

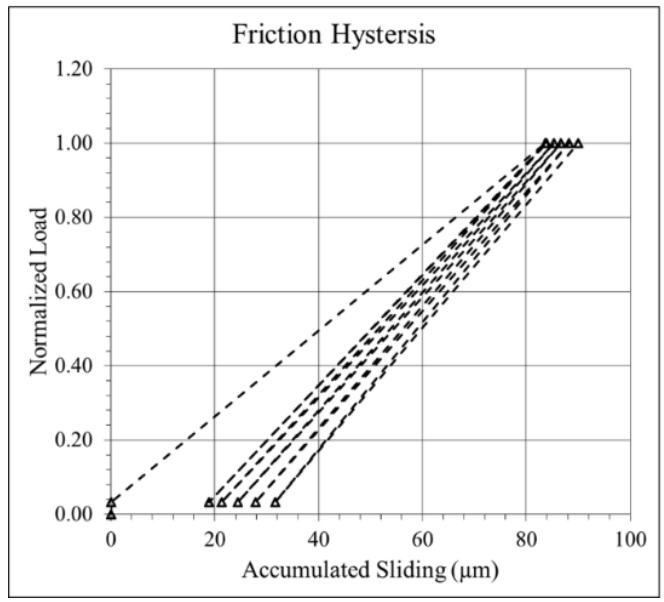

(b)

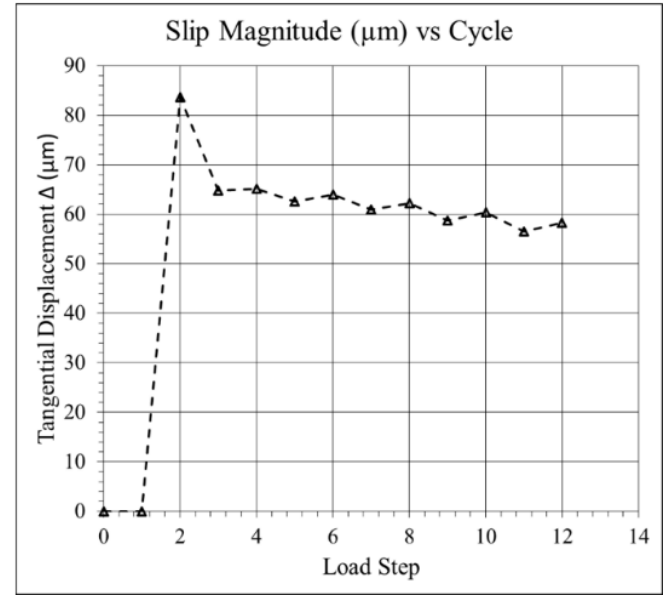

Fig. 87. (a) Friction hysteresis at edge of contact (b) Slip magnitude for COF value of 0.70
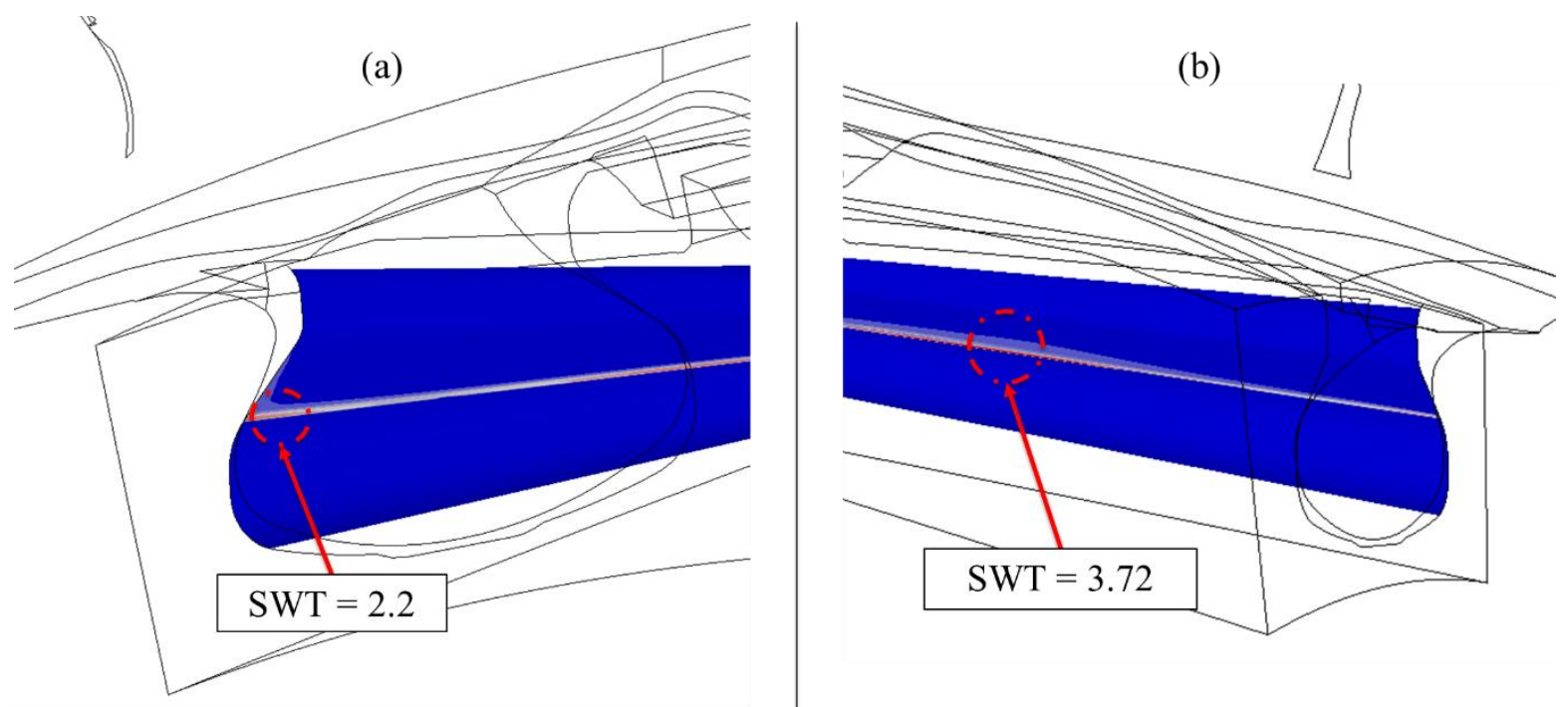

Fig. 88. SWT parameter calculated at (a) Pressure side and (b) Suction side 

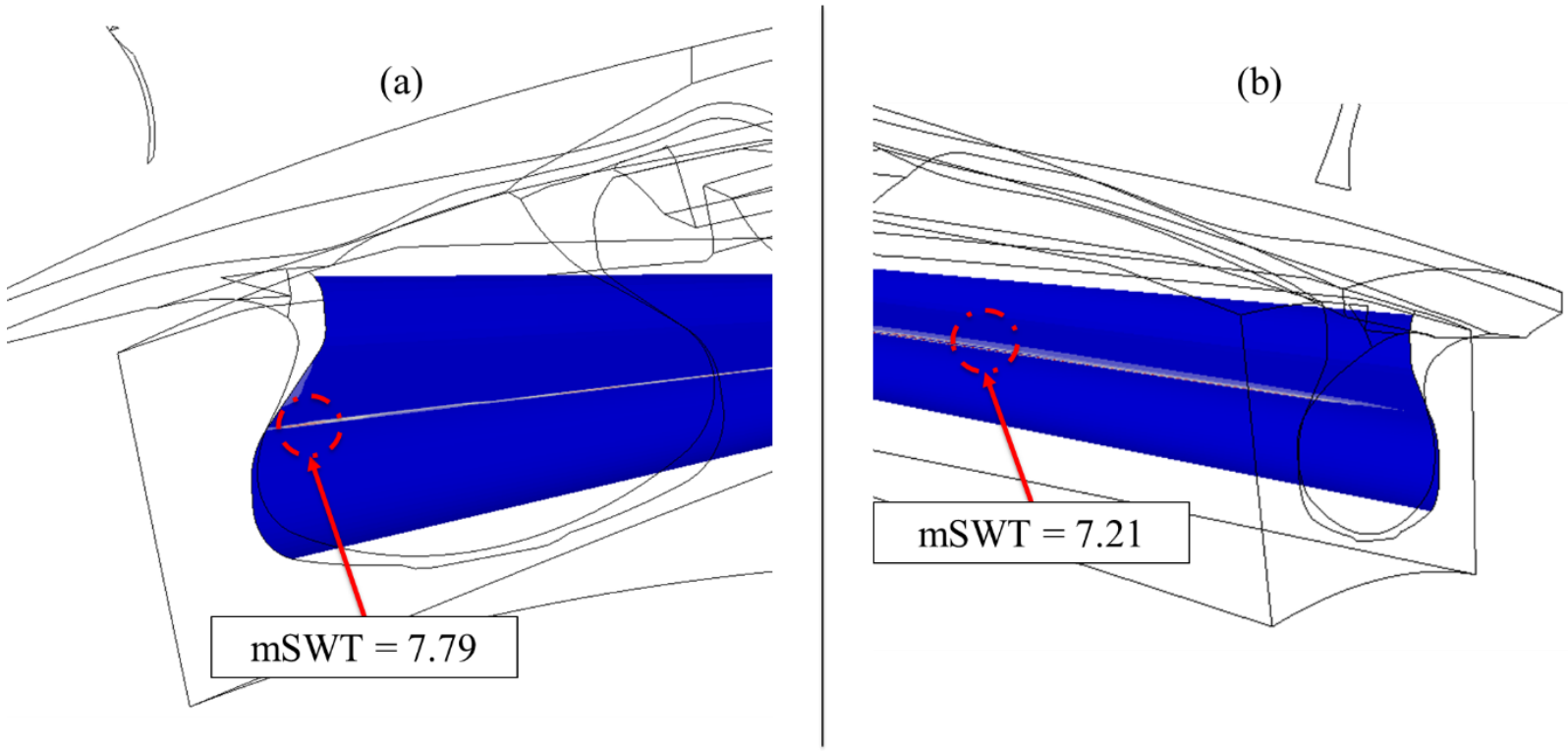

Fig. 89. Modified SWT parameter calculated at (a) Pressure side and (b) Suction side

(a)

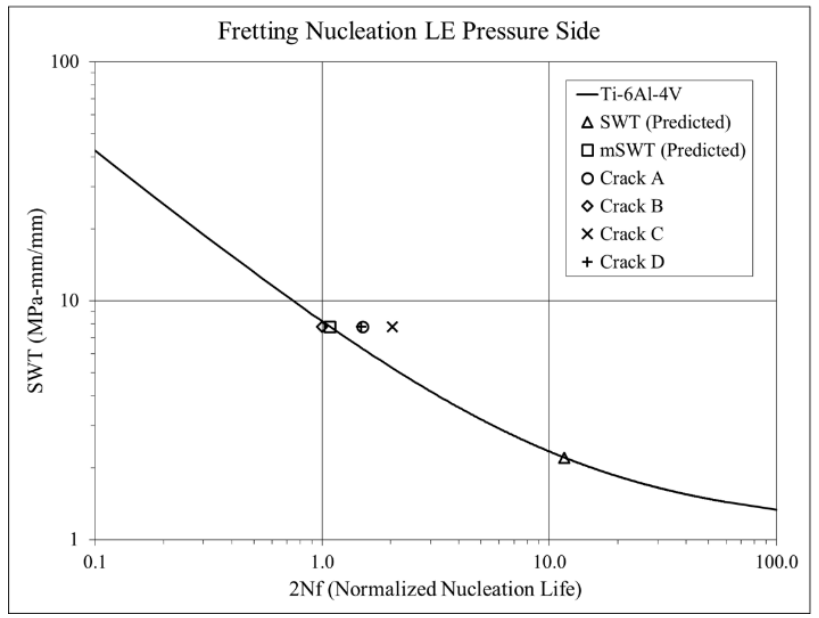

(b)

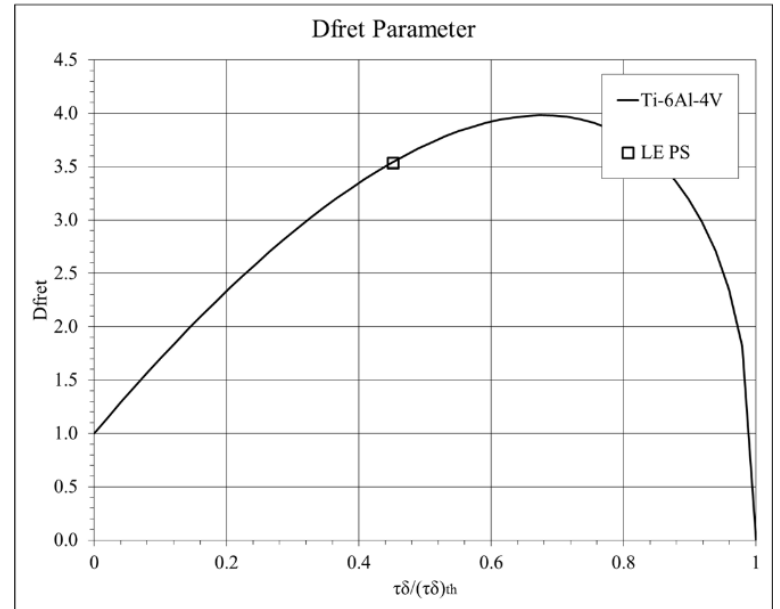

Fig. 90. (a) Fretting nucleation life calculated at the critical LE pressure side location (b) $D_{\text {fret }}$ curve 


\subsection{FINDINGS}

1. The fretting fatigue behavior of a Ti-6Al-4V dovetail fan has been modeled. A fracture mechanics based approach was used to obtain an evolved COF at the fan blade and hub interface. The evolution considers both nucleation and propagation phases of the fretting crack.

2. $3 \mathrm{D}$ crack growth simulations were conducted for the fan dovetail with two unique COF values $(0.35,0.70)$ at the contact interface. The simulation corresponding to a COF value of 0.70 resulted in a crack trajectory, aspect ratio, shape, and propagation life which matched more closely with the fractured components. This $\mathrm{COF}$ value was therefore used to predict the fretting fatigue of the fan hub.

3. The fretting fatigue of the fan hub was assessed using the plain fatigue SWT model and modified SWT model. The fretting specific modified SWT parameter more accurately predicted the nucleation location of the crack and nucleation life compared to the plain fatigue SWT parameter.

4. Since the tests presented only a single calibration criterion, the obtained $\mathrm{D}_{\text {fret }}$ parameters naturally produced a fretting fatigue life identical to test results. The validity of the approach needs to be further checked at different loading conditions and other fan dovetail attachments. 


\section{CONCLUSION}

The fatigue and fracture for bladed and integrally bladed rotors of aircraft engine compressors has been numerically modeled through a damage tolerant approach with the aim of accurately determining the crack propagation life, trajectory, and the size and shape of the fatigue cracks in comparison to collected field experience and test data.

Compressor rotors in operation typically exhibit two identified regimes of crack growth. The first is a LCF growth regime, where the minor cycles associated with HCF loading do not contribute to the advancement of the crack and have negligible crack growth rate. The second is a HCF crack growth onset regime, where a LCF driven crack grows large enough so that the applied stress intensity under resonant vibration is greater than the materials threshold stress intensity. Depending on the type of rotor, the location of crack nucleation, and engine application, the influence of HCF to overall crack growth behavior may or may not be significant. For instance, bladed rotors are often life limited at attachment locations due to fretting fatigue, in which a crack nucleates from HCF but propagates under LCF given that the vibratory stresses in the bulk of the disk slot are sufficiently damped. However, studies have shown that when present, the influence of vibratory stresses on the direction and shape of the crack could be significant such as a crack on an airfoil of a compressor IBR where resonant vibration has pronounced effects. In current literature, there is uncertainty about the actual vibratory stress levels experienced by components and a lack of studies devoted to the numerical modeling of crack growth under mixed non-proportional combined LCF-HCF loading of compressor blades. In Chapter 3, a new numerical technique was presented which was used to combine steady and dynamic stresses to simulate 3D crack growth of IBR compressor blades when subjected to HCF-LCF mixed non proportional loads. The method was applied on a compressor IBR which fractured in service caused by the excitation of the compressor airfoils' first bending mode. The results from the simulation agreed well with the fractured airfoils after the combined stress field was considered. The technique can be extended on other compressor disk applications to enhance damage tolerant design methodologies. For crack propagation life, the linear summation model was used to account for both LCF and HCF cycles. It was concluded that the effects of LCF major and minor cycles to overall crack growth was insignificant compared to the contribution of HCF loading. Although HCF is the primary mechanism for the final fracture, LCF loading accelerates crack growth by imposing a positive mean stress to the alternating HCF dynamic stresses. The method presented in Chapter 3 requires an iterative process of applying tip bending forces to produce a stress distribution and tip deflection equivalent to the modal solution and NSMS measured tip deflections. For higher order modes including but not limited to airfoil torsional modes, this iterative process could present analysts with a more difficult challenge. For future work, perhaps an automated or closed form solution can be explored to more efficiently superimpose LCF with HCF loading, allowing for crack growth modeling under higher order modes.

For blade/disk assemblies of bladed compressors, literature has suggested that one of the controlling factors for life is fretting fatigue. Fretting, which is the relative tangential motion $\delta$ occurring between clamped bodies in contact when subjected to oscillatory forces can significantly reduce the fatigue performance of materials relative to their plain fatigue limits. Therefore, fretting should be accounted for in the lifing methodologies of bladed rotors of aircraft engines. However, damage caused by fretting is difficult to model and predict since both fatigue and wear occur concurrently with competing effects. Earlier modeling attempts which can be found in literature today focus mainly 
on conditions where one of the damage mechanisms, namely fatigue, is dominant, while ignoring secondary damage considerations attributed to wear. As such, strain-based fatigue and critical plane methodologies such as the SWT model have been successfully used in the past to determine fretting crack nucleation life as well as the location of the fretting nucleated crack for Ti-6Al-4V titanium alloys. These critical plane approaches although previously used successfully, lack a secondary damage mechanism attributed to fretting wear, and thus in some cases, could misrepresent the true fretting fatigue phenomenon. More recent literature suggests the use of the modified SWT parameter, which takes into account both damage mechanisms to predict fretting nucleation. Furthermore, the evolution of the COF due to fretting, an important contributor to fretting fatigue behavior, is often ignored in lifing methodologies. For the dovetail fan attachment studied in this work, the COF is minimum at initial assembly when the parts are undamaged with the presence of surface oxide films on the contact surfaces. When the oxide films are removed due to mechanical wear resulting from fretting, the underlying metals of the contact surfaces adhere, resulting in a strong increase to the COF. In Chapter 4, a fracture mechanics based approach was developed and used to model the fretting fatigue behavior of a fan dovetail attachment. A crack propagation analysis was conducted to obtain an 'average' value of COF to characterize the evolution of the COF in the nucleation and propagation phases of the fretting crack. The fretting fatigue behavior was modeled with the obtained COF using the plain fatigue SWT parameter and the fretting-specific modified SWT parameter. The predicted nucleation location, nucleation life, crack trajectory, shape and propagation life obtained from the method agreed well with the fractured components. The study also confirmed that the recently developed fretting-specific modified SWT parameter more accurately predicts the nucleation location and life of the crack compared to the plain fatigue SWT parameter. For future work, the validity of the approach needs to be further investigated for different loading conditions and other fan dovetail attachments. 


\section{REFERENCES}

[1] Wikipedia. [Online]. Available: https://en.wikipedia.org/wiki/Turbofan\#/media/File:Turbofan_operation.svg. [Accessed 24 February 2018].

[2] D. Greatrix, Powered Flight: The Engineering of Aerospace Propulsion, 1st Edition, Springer-Verlag London, 2012.

[3] K. Anandavel and R. V. Prakash, "Effect of three-dimensional loading on macroscopic fretting aspects of an aero-engine blade-disc dovetail interface," Tribology International, vol. 44, no. 11, pp. 1544-1555, 2011.

[4] M. Hawkyard, B. Powell, J. Stephenson and M. McElhone, "Fatigue crack growth from simulated flight cycles involving superimposed vibrations," International Journal of Fatigue, vol. 21, pp. S59-S68, 1999.

[5] B. Cowles, "High cycle fatigue in aircraft gas turbines - an industry perspective," International Journal of Fracture, vol. 80, pp. 147-163, 1996.

[6] J. Byrne, R. Hall and B. Powell, "Influence of LCF overloads on combined HCF/LCF crack growth," International Journal of Fatigue, vol. 25, pp. 827-834, 2003.

[7] B. Cowles, "Life Prediction in High Temperature Environments: Overview of a Current Gas Turbine Engine Approach," Materials Science and Engineering, vol. 103, pp. 63-69, 1988.

[8] K. Barlow and R. Chandra, "Fatigue crack propagation simulation in an aircraft engine fan blade attachment," International Journal of Fatigue, vol. 27, no. 10-12, pp. 1661-1668, 2005.

[9] L. Witek, "Simulation of crack growth in the compressor blade subjected to resonant vibration using hybrid method," Engineering Failure Analysis, vol. 49, pp. 57-66, 2015.

[10] R. Ritchie, B. Boyce, J. Campbell, 0. Roder, A. Thompson and W. Milligan, "Thresholds for high-cycle fatigue in a turbine engine Ti-6Al-4V alloy," International Journal of Fatigue, vol. 21, no. 7, pp. 653-662, 1999.

[11] R. Pettit, B. Annigeri, W. Owen and P. Wawrzynek, "Next generation 3D mixed mode fracture propagation theory including HCF-LCF interaction," Engineering Fracture Mechanics, vol. 102, pp. 1-14, 2013.

[12] D. Mangardich, F. Abrari and Z. Fawaz, "Modeling crack growth of an aircraft engine high pressure compressor blade under combined HCF and LCF loading," Engineering Fracture Mechanics, vol. 214, no. C, pp. 474-486, 2019.

[13] D. Hills, "Mechanics of fretting fatigue," Wear, vol. 175, no. 1-2, pp. 107-113, 1994.

[14] M. P. Szolwinski and T. N. Farris, "Mechanics of fretting fatigue crack formation," Wear, vol. 198, no. 1-2, pp. 93-107, 1996.

[15] O. Jin. and S. Mall, "Effects of slip on fretting behavior: experiments and analyses," Wear, vol. 256, no. 7-8, pp. 671-684, 2004.

[16] M. Hirsch, "Temperature Dependent Fretting Damage Modeling of AISI 301 Stainless Steel," Georgia Institute of Technology, 2013. 
[17] J. Ding, S. Leen and I. McColl, "The effect of slip regime on fretting wear-induced stress evolution," International Journal of Fatigue, vol. 26, no. 5, pp. 521-531, 2004.

[18] B. Carter, E. Schenck, P. Wawrzynek, A. Ingraffea and K. Barlow, "Three-dimensional simulation of fretting crack nucleation and growth," Engineering Fracture Mechanics, vol. 96, pp. 447-460, 2012.

[19] T. Nicholas, "Critical issues in high cycle fatigue," International Journal of Fatigue, vol. 21, no. Supplement 1, pp. S221-S231, 1999.

[20] B.-W. Lee, J. Suh, H. Lee and T.-g. Kim, "Investigations on fretting fatigue in aircraft engine compressor blade," Engineering Failure Analysis, vol. 18, no. 7, pp. 1900-1908, 2011.

[21] H. Tang, D. Cao, H. Yao, M. Xie and R. Duan, "Fretting fatigue failure of an aero engine turbine blade," Engineering Failure Analysis, vol. 16, no. 6, pp. 2004-2008, 2009.

[22] S. Barella, M. Boniardi, S. Cincera, P. Pellin, X. Degive and S. Gijbels, "Failure analysis of a third stage gas turbine blade," Engineering Failure Analysis, vol. 18, no. 1, pp. 386-393, 2011.

[23] K. Smith, W. P. and T. T.H., "A stress-strain function for the fatigue of metals," Journal of Materials, vol. 5, pp. $767-778,1970$.

[24] C. Lykins, S. Mall and V. Jain, "A shear stress-based parameter for fretting fatigue crack initiation," Fatigue \& Fracture of Engineering Materials \& Structures, vol. 24, no. 7, pp. 461-473, 2001.

[25] J. Araujo, D. Nowell and R. Vivacqua, "The use of multiaxial fatigue models to predict fretting fatigue life of components subjected to different contact," Fatigue \& Fracture of Engineering Materials \& Structures, vol. 27, no. 10, pp. 967-978, 2004.

[26] C. D. Lykins, S. Mall and V. Jain, "An evaluation of parameters for predicting fretting fatigue crack initiation," International Journal of Fatigue, vol. 22, no. 8, pp. 703-716, 2000.

[27] W. Siang Sum, E. J. Williams and S. B. Leen, "Finite element, critical-plane, fatigue life prediction of simple and complex contact configurations," International Journal of Fatigue, vol. 27, no. 4, pp. 403-416, 2005.

[28] V. Fridrici, S. Fouvry, P. Kapsa and P. Perruchaut, "Prediction of cracking in Ti-6Al-4V alloy under frettingwear: use of the SWT criterion," Wear, vol. 259, no. 1-6, pp. 300-308, 2005.

[29] A. Fatemi and D. F. Socie, "A Critical Plane Approach to Multiaxial Fatigue Damage Including Out-Of-Phase Loading," Fatigue \& Fracture of Engineering Materials \& Structures, vol. 11, no. 3, pp. 149-165, 1988.

[30] C. Ruiz, P. Boddington and K. Chen, "An Investigation of Fatigue and Fretting in a Dovetail Joint," Experimental Mechanics, vol. 24, no. 3, pp. 208-217, 1984.

[31] O. Vingsbo and S. Soderberg, "On Fretting Maps," Wear, vol. 126, no. 2, pp. 131-147, 1988.

[32] D. Nowell, D. Dini and D. Hills, "Recent developments in the understanding of fretting fatigue," Engineering Fracture Mechanics, vol. 73, no. 2, pp. 207-222, 2006.

[33] J. Ding, W. Sum, R. Sabesan, S. Leen, I. McColl and E. Williams, "Fretting fatigue predictions in a complex coupling," International Journal of Fatigue, vol. 29, no. 7, pp. 1229-1244, 2007. 
[34] I. McColl, J. Ding and S. Leen, "Finite element simulation and experimental validation of fretting wear," Wear, vol. 256, no. 11-12, pp. 1114-1127, 2004.

[35] D. Mangardich, F. Abrari and Z. Fawaz, "A fracture mechanics based approach for the fretting fatigue of aircraft engine fan dovetail attachments," International Journal of Fatigue, vol. 10.1016/j.ijfatigue.2019.105213, 2019.

[36] D. Broek, Elementary engineering fracture mechanics, Sijthoff \& Noordhoff International Publishers B.C., Alphen aan den Rijn, The Netherlands, 1978.

[37] T. Anderson, Fracture Mechanics Fundamentals \& Applications, Fourth Edition, CRC Press Taylor \& Francis Group, LLC, 2017.

[38] D. Dugdale, "Yielding of steel sheets containing slits," Journal of the Mechanics and Physics of Solids, 1960.

[39] A. Varvani, "Fatigue Fracture Analysis," in ME8136: Advanced Fatigue Fracture Analysis, Ryerson University, 2017.

[40] J. Glen R. Romanoski, "The Fatigue Behaviour of Small Cracks in Aircraft Turbine Disk Alloys," Massachusetts Institute of Technology, 1990.

[41] J. Newman Jr, E. Phillips and M. Swain, "Fatigue-life prediction methodology using small-crack theory," International Journal of Fatigue, 1999.

[42] A. Vasudeven, K. Sadananda and N. Louat, "A review of crack closure, fatigue crack threshold and related phenomena," Materials" Science and Engineering, 1994.

[43] W. Elber, "Fatigue Crack Closure Under Cyclic Tension," Engineering Fracture Mechanics, 1970.

[44] J. Newman, "A Crack-Closure model for predicting fatigue-crack growth under aircraft spectrum loading," ASTM, 1981.

[45] H. Doker and G. Marci, "Threshold range and opening stress intensity factor in fatigue," International Journal of Fatigue, 1983.

[46] B. Boyce and R. Ritchie, "Effect of load ratio and maximum stress intensity on the fatigue threshold in Ti-6Al4V," Engineering Fracture Mechanics, 2001.

[47] M. Skorupa, T. Machniewicz, J. Schijve and A. Skorupa, "Application of the strip-yield model from the NASGRO software to predict fatigue crack growth in aluminium alloys under constant and variable amplitude loading," Engineering Fracture Mechanics, vol. 74, no. 3, pp. 291-313, 2007.

[48] F. A. C. Inc, FRANC3D Reference Manual Version 7.1, 2017.

[49] J. Rice, "A Path Independent Integral and the Approximate Analysis of Strain Concentration by Notches and Cracks," Department of Defense Advanced Research Projects Agency Contract SD-86 Material Research Program, 1967.

[50] P. Wawrzynek, B. Carter and L. Banks-Sills, "The M-Integral for Computing Stress Intensity Factors in Generally Anisotropic Materials," Prepared for Marshall Space Flight Center under Contract NAS8-36801 and sponsored by the Space Shuttle Main Engine Program managed at the Marshall Space Flight Center, 2005. 
[51] P. Bold, M. Brown and R. Allen, "A review of fatigue crack growth in steels under mixed mode I and II loading," Fatigue of Engineering Materials Ltd, 1992.

[52] Y. J. Chao and S. Liu, "On the failure of cracks under mixed-mode loads," International Journal of Fracture, 1997.

[53] R. Nuismer, "An energy release rate criterion for mixed mode fracture," International Journal of Fracture, 1975.

[54] A. Kfouri and M. Brown, "A Fracture Criterion for Cracks under Mixed-Mode Loading," Fatigue \& Fracture of Engineering Materials \& Structures, 1995.

[55] P. Wawrzynek and A. Ingraffea, "Interactive finite element analysis of fracture processes: An integrated approach," Theoretical and Applied Fracture Mechanics, vol. 8, pp. 137-150, 1987.

[56] I. Fracture Analysis Consultants, "FRANC3D Version 7," [Online]. Available: http://www.fracanalysis.com/pdfs/FRANC3D\%20V7.1\%20Brochure.pdf. [Accessed 10 February 2018].

[57] F. A. C. Inc, Benchmark Manual Version 7.1, 2017.

[58] R. Rajasekaran and D. Nowell, "Fretting fatigue in dovetail blade roots: Experiment and analysis," Tribology International, vol. 39, no. 10, pp. 1277-1285, 2006.

[59] D. Swalla and R. Neu, "Influence of coefficient of friction on fretting fatigue crack nucleation prediction," Tribology International, vol. 34, no. 7, pp. 493-503, 2001.

[60] D. Garcia and A. Grandt Jr., "Fractographic investigation of fretting fatigue cracks in Ti-6Al-4V," Engineering Failure Analysis, vol. 12, no. 4, pp. 537-548, 2005.

[61] A. Hutson, C. Neslen and T. Nicholas, "Characterization of fretting fatigue crack initiation processes in CR Ti6Al-4V," Tribology International, 2003.

[62] P. J. Golden, "International Journal of Fatigue," Development of a dovetail fretting fatigue fixture for turbine engine materials, 2009.

[63] P. Golden and J. Calcaterra, "A fracture mechanics life prediction methodology applied to dovetail fretting," Tribology International, vol. 39, no. 10, pp. 1172-1180, 2006.

[64] S. Fouvry and K. Kubiak, "Introduction of a fretting-fatigue mapping concept: Development of a dual crack nucleation - crack propagation approach to formalize fretting-fatigue damage," International Journal of Fatigue, 2009.

[65] H. Fadag, S. Mall and V. Jain, "A finite element analysis of fretting fatigue crack growth behavior in Ti-6Al4V," Engineering Fracture Mechanics, vol. 75, no. 6, pp. 1384-1399, 2008.

[66] E. Hershko, N. Mandelker, G. Gheorghiu, H. Sheinkopf, I. Cohen and O. Levy, "Assessment of fatigue striation counting accuracy using high resolution scanning electron microscope," Engineering Failure Analysis, vol. 15, pp. 20-27, 2008.

[67] E. N. Cherolis, "Fatigue in the Aerospace Industry: Striations," Journal of Failure Analysis and Prevention, vol. 8, pp. 255-258, 2008. 
[68] T. Nicholas, "Chapter 4: LCF-HCF Interactions," in High Cycle Fatigue - A Mechanics of Material Perspective, Ohio, Elsevier, 2006.

[69] M. Hawkyard, B. Powell, I. Hussey and L. Grabowski, "Fatigue Crack Growth Under The Conjoint Action of Major \& Minor Stress Cycles," Fatigue \& Fracture of Engineering Materials \& Structures Ltd, vol. 19, pp. 217-227, 1996.

[70] B. Powell, T. Duggan and R. Jeal, "The influence of minor cycles on low cycle fatigue crack propagation," International Journal of Fatigue, vol. 4, pp. 4-14, 1982.

[71] B. Powell, M. Hawkyard and L. Grabowski, "The growth of cracks in Ti-6Al-4V plate under combined high and low cycle fatigue," International Journal of Fatigue, vol. 19, pp. 167-176, 1997.

[72] R. Hall and B. Powell, "Crack growth in IMI 829 at $550^{\circ} \mathrm{C}$ under combined high and low cycle fatigue," Materials at High Temperatures, vol. 19, pp. 1-8, 2002.

[73] C. Schweizer, T. Nieweg, B. Nieweg, P. von Hartrott and H. Riedel, "Mechanisms and modelling of fatigue crack growth under combined low and high cycle fatigue loading," International Journal of Fatigue, vol. 33, pp. 194-202, 2011.

[74] S. Soderberg, U. Bryggman and T. McCullough, "Frequency Effects in Fretting Wear," Wear, vol. 110, no. 1, pp. 19-34, 1986.

[75] P. Golden, "Development of a dovetail fretting fatigue fixture for turbine engine materials," International Journal of Fatigue, vol. 4, no. 31, pp. 620-628, 2009.

[76] P. Golden and A. Grandt Jr., "Fracture mechanics based fretting fatigue life predictions in Ti-6Al-4V," Engineering Fracture Mechanics, vol. 71, no. 15, pp. 2229-2243, 2004.

[77] C. D. , "An investigation of fretting fatigue crack initiation behavior of the titanium alloy Ti-6Al-4V," Thesis (Ph.D.)--University of Dayton, p. 136, 1999. 